\title{
Evolutionary branching under multi-dimensional evolutionary constraints
}

\author{
Hiroshi C. Ito $\left(1,{ }^{*}\right)$ and Akira Sasaki $(1,2)$ \\ 1) Department of Evolutionary Studies of Biosystems, SOKENDAI (The \\ Graduate University for Advanced Studies, Hayama, Kanagawa 240-0193, \\ Japan \\ 2) Evolution and Ecology Program, International Institute for Applied Systems \\ Analysis, Laxenburg, Austria \\ * Corresponding author: (hiroshibeetle@gmail.com)
}




\section{Abstract}

The fitness of an existing phenotype and of a potential mutant should generally depend on the frequencies of other existing phenotypes. Adaptive evolution driven by such frequency-dependent fitness functions can be analyzed effectively using adaptive dynamics theory, assuming rare mutation and asexual reproduction. When possible mutations are restricted to certain directions due to developmental, physiological, or physical constraints, the resulting adaptive evolution may be restricted to subspaces (constraint surfaces) with fewer dimensionalities than the original trait spaces. To analyze such dynamics along constraint surfaces efficiently, we develop a Lagrange multiplier method in the framework of adaptive dynamics theory. On constraint surfaces of arbitrary dimensionalities described with equality constraints, our method efficiently finds local evolutionarily stable strategies, convergence stable points, and evolutionary branching points. We also derive the conditions for the existence of evolutionary branching points on constraint surfaces when the shapes of the surfaces can be chosen freely.

\section{Introduction}

Individual organisms have many traits undergoing selection simultaneously, inducing their simultaneous evolution. At the same time, evolutionary constraints (i.e., trade-offs) often exist, such that a mutation improving one trait inevitably makes another trait worse (Flatt and Heyland, 2011), e.g., trade-off between speed and efficiency in feeding activity of a zooplankton species (Daphnia dentifera) (Hall et al., 2012). In those cases, the second trait may be treated as a function of the first trait. In such a manner, evolution of populations in multi-dimensional trait spaces may be restricted to subspaces with fewer dimensionalities. We call such subspaces 'constraint surfaces' for convenience, although they may be one dimensional (curves), two dimensional (surfaces), or multi-dimensional (hypersurfaces).

In adaptive dynamics theory (Metz et al., 1996; Dieckmann and Law, 1996), directional evolution along such a constraint surface can be analyzed easily by examining selection pressures tangent to the surface, which allows us to find evolutionarily singular points where directional selection along the surface vanishes (deMazancourt and Dieckmann, 2004; Parvinen et al., 2013). On the other hand, evolutionary stability (Maynard Smith, 1982) and convergence stability (Eshel, 1983) of those singular points can be affected by the local curvature of the 
surface. At present, analytical methods for examining both evolutionary and convergence stabilities have been developed for one-dimensional constraint curves in two-dimensional trait spaces (deMazancourt and Dieckmann, 2004; Kisdi, 2006) and in arbitrary higher-dimensional trait spaces (Kisdi, 2015). In this paper, we develop a Lagrange multiplier method that allows us to analyze adaptive evolution along constraint surfaces of arbitrary dimensionalities in trait spaces of arbitrary dimensionalities, as if no constraint exists. We focus on evolutionary branching points (points that are convergence stable but evolutionarily unstable), which induce evolutionary diversification through a continuous process called evolutionary branching (Geritz et al., 1997). Points of other kinds defined by combinations of evolutionary stability and convergence stability (e.g., points that are locally evolutionarily stable as well as convergence stable) can be analyzed in the same manner.

This paper is structured as follows. Section 2 contains a brief explanation of the basic assumptions of adaptive dynamics theory and a standard analysis of adaptive evolution along constraint surfaces. Section 3 presents the main mechanism of our method in the case of one-dimensional constraint curves in twodimensional trait spaces. In section 4, we describe a general form of our method for an arbitrary $L$-dimensional constraint surface embedded in an arbitrary $M$ dimensional trait space. In section 5 , the conditions for existence of candidate branching points (CBPs) along constraint surfaces when their shapes can be chosen freely are derived. Section 6 shows two simple application examples. In section 7 , we discuss our method in relation to other studies.

\section{Basic assumptions and motivation}

To analyze evolutionary dynamics, we use adaptive dynamics theory (Metz et al., 1996; Dieckmann and Law, 1996). For simplicity, we consider a single asexual population in a two-dimensional trait space $\mathbf{s}=(x, y)^{\mathrm{T}}$ with two scalar traits $x$ and $y$, in which all possible mutants $\mathbf{s}^{\prime}=\left(x^{\prime}, y^{\prime}\right)^{\mathrm{T}}$ are restricted to a constraint curve $h\left(\mathbf{s}^{\prime}\right)=0$. The theory (sensu stricto) assumes sufficiently rare mutations and a sufficiently large population size, so that the population is monomorphic and almost at equilibrium density whenever a mutant emerges. In this case, whether a mutant can invade the resident population can be determined by the mutant's initial per capita growth rate, called invasion fitness $F\left(\mathbf{s}^{\prime} ; \mathbf{s}^{\circ}\right)$, which is a function of the mutant phenotype $\mathbf{s}^{\prime}$ and the resident phenotype $\mathbf{s}^{\circ}=\left(x^{\circ}, y^{\circ}\right)^{\mathrm{T}}$. The mutant can invade the resident only when $F\left(\mathbf{s}^{\prime} ; \mathbf{s}^{\circ}\right)$ is positive, resulting in substitution of the resident in many cases. Repetition of such a substitution is 
called a trait substitution sequence, forming directional evolution toward greater fitness as long as the fitness gradient at the resident is not small. Under certain conditions, when the fitness gradient along the curve becomes small, a mutant may coexist with the resident, which may bring about evolutionary diversification into two distinct morphs, called evolutionary branching (Metz et al., 1996; Geritz et al., $1997,1998)$. In this paper, we assume for simplicity that the population is unstructured, although our results (Theorems 1-3) are also applicable to structured populations, as long as reproduction is asexual and the invasion fitness function is defined in the form of $F\left(\mathbf{s}^{\prime} ; \mathbf{s}^{\circ}\right)$.

Denoting points on the constraint curve by $\mathbf{s}(\phi)$ with a scalar parameter $\phi$, we can express the resident and mutant phenotypes as $\mathbf{s}^{\circ}=\left(x\left(\phi^{\circ}\right), y\left(\phi^{\circ}\right)\right)^{\mathrm{T}}$ and $\mathbf{s}^{\prime}=\left(x\left(\phi^{\prime}\right), y\left(\phi^{\prime}\right)\right)^{\mathrm{T}}$, respectively. In this case, the evolutionary dynamics along the curve can be translated into that in a one-dimensional trait space $\phi$. The expected shift of the resident phenotype due to directional evolution can be described by an ordinary differential equation (Dieckmann and Law 1996):

$$
\frac{\mathrm{d} \phi^{\circ}}{\mathrm{d} t}=\frac{n^{\circ} \mu \sigma_{\mu}^{2}}{2} g\left(\phi^{\circ}\right),
$$

where $n^{\circ}$ is the equilibrium population density for a monomorphic population of $\mathbf{s}^{\circ}, \mu$ is the mutation rate per birth, $\sigma_{\mu}$ is the root mean square of mutational steps $\phi^{\prime}-\phi^{\circ}$, and

$$
g\left(\phi^{\circ}\right)=\left[\frac{\partial F\left(\mathbf{s}^{\prime} ; \mathbf{s}^{\circ}\right)}{\partial \phi^{\prime}}\right]_{\phi^{\prime}=\phi^{\circ}}
$$

is the fitness gradient along the curve at the position where the resident exists (Eq. (1a) is specific to unstructured populations; see also Durinx et al. (2008) for a general form for structured populations). Here, $n^{\circ}, \mu$, and $\sigma_{\mu}$, as well as $g\left(\phi^{\circ}\right)$, may depend on $\phi^{\circ}$, although they are denoted without $\left(\phi^{\circ}\right)$ for convenience. In adaptive evolution, along the parameterized constraint curve, the conditions for evolutionary branching are identical to those for one-dimensional trait spaces without constraint (Metz et al., 1996; Geritz et al., 1997). Specifically, along the constraint curve, a point $\mathbf{s}=(x(\phi), y(\phi))^{\mathrm{T}}$ is an evolutionary branching point, if it is (i) evolutionarily singular,

$$
g(\phi)=0
$$

(i.e., no directional selection for a population located at s), (ii) convergence stable (Eshel, 1983), 


$$
C(\phi) \equiv\left[\frac{\partial g\left(\phi^{\circ}\right)}{\partial \phi^{\circ}}\right]_{\phi^{\circ}=\phi}<0
$$

(i.e., $\mathbf{s}$ is a point attractor in terms of directional selection), and (iii) evolutionarily unstable (Maynard Smith, 1982),

$$
D(\phi) \equiv\left[\frac{\partial^{2} F\left(\mathbf{s}^{\prime} ; \mathbf{s}^{\circ}\right)}{\partial \phi^{\prime 2}}\right]_{\phi^{\prime}=\phi^{\circ}=\phi}>0
$$

(i.e., for residents, $\mathbf{s}^{\circ}=\mathbf{s}, F\left(\mathbf{s}^{\prime} ; \mathbf{s}\right)$ forms a fitness valley along $\mathbf{s}^{\prime}$ with its bottom $\mathbf{s}^{\prime}=\mathbf{s}$ leading to disruptive selection). Eq. (2b) can be expressed alternatively by noting (1b) as

$$
C(\phi)=\left[\frac{\partial}{\partial \phi^{\circ}}\left(\frac{\partial F\left(\mathbf{s}^{\prime} ; \mathbf{s}^{\circ}\right)}{\partial \phi^{\prime}}\right)_{\phi^{\prime}=\phi^{\circ}}\right]_{\phi^{\circ}=\phi}=D(\phi)+\left[\frac{\partial^{2} F\left(\mathbf{s}^{\prime} ; \mathbf{s}^{\circ}\right)}{\partial \phi^{\prime} \partial \phi^{\circ}}\right]_{\phi^{\prime}=\phi^{\circ}=\phi}<0 .
$$

However, in trait spaces with more than two dimensions, constraints may form surfaces or hyper-surfaces whose parametric expression may be difficult or complicated. To avoid such difficulty, we develop an alternative approach that does not require parametric expression of constraint spaces.

\section{One-dimensional constraint curves in two- dimensional trait spaces}

The Lagrange multiplier method is a powerful tool for finding local maxima and minima of functions that are subject to equality constraints. In this section, we develop a method for adaptive dynamics under constraints in the form of Lagrange multiplier method. For clarity, we consider the simplest case: constraint curves $h\left(\mathbf{s}^{\prime}\right)=0$ in two-dimensional trait spaces $\mathbf{s}=(x, y)^{\mathrm{T}}$. The method is generalized to arbitrary dimensions in the subsequent section.

\subsection{Notations for derivatives}

For convenience, we introduce some notations for derivatives of functions by their vector arguments. For a function with a single vector argument, its derivative by that argument is denoted by $\nabla$. For a function with more than one argument, its partial derivative by its argument $\mathbf{z}$ is denoted by $\nabla_{\mathbf{z}}$. The same rule applies for second derivatives. We express first and second derivatives of the constraint 
function $h\left(\mathbf{s}^{\prime}\right)$ and the fitness function $F\left(\mathbf{s}^{\prime} ; \mathbf{s}^{\circ}\right)$ (at an arbitrary point $\mathbf{s}$ ) as follows. For $h\left(\mathbf{s}^{\prime}\right)$, we write the gradient and its transpose as

$$
\begin{aligned}
& \nabla h(\mathbf{s})=\left(\begin{array}{l}
\frac{\partial h\left(\mathbf{s}^{\prime}\right)}{\partial x^{\prime}} \\
\frac{\partial h\left(\mathbf{s}^{\prime}\right)}{\partial y^{\prime}}
\end{array}\right)_{\mathbf{s}^{\prime}=\mathbf{s}}, \\
& \nabla^{\mathrm{T}} h(\mathbf{s})=\left(\frac{\partial h\left(\mathbf{s}^{\prime}\right)}{\partial x^{\prime}} \frac{\partial h\left(\mathbf{s}^{\prime}\right)}{\partial y^{\prime}}\right)_{\mathbf{s}^{\prime}=\mathbf{s}},
\end{aligned}
$$

and the Hessian matrix as

$$
\nabla \nabla^{\mathrm{T}} h(\mathbf{s})=\left(\begin{array}{ll}
\frac{\partial^{2} h\left(\mathbf{s}^{\prime}\right)}{\partial x^{\prime 2}} & \frac{\partial^{2} h\left(\mathbf{s}^{\prime}\right)}{\partial x^{\prime} \partial y^{\prime}} \\
\frac{\partial^{2} h\left(\mathbf{s}^{\prime}\right)}{\partial x^{\prime} \partial y^{\prime}} & \frac{\partial^{2} h\left(\mathbf{s}^{\prime}\right)}{\partial y^{\prime 2}}
\end{array}\right)_{\mathbf{s}^{\prime}=\mathbf{s}} .
$$

For the fitness function $F\left(\mathbf{s}^{\prime} ; \mathbf{s}^{\circ}\right)$, we write the first and second derivatives by $\mathbf{s}^{\prime}$ at the position where $\mathbf{s}^{\circ}$ exists as

$$
\begin{gathered}
\nabla_{\mathbf{s}^{\prime}} F\left(\mathbf{s}^{\circ} ; \mathbf{s}^{\circ}\right)=\left(\begin{array}{l}
\frac{\partial F\left(\mathbf{s}^{\prime} ; \mathbf{s}^{\circ}\right)}{\partial x^{\prime}} \\
\frac{\partial F\left(\mathbf{s}^{\prime} ; \mathbf{s}^{\circ}\right)}{\partial y^{\prime}}
\end{array}\right)_{\mathbf{s}^{\prime}=\mathbf{s}^{\circ}}=\mathbf{g}\left(\mathbf{s}^{\circ}\right), \\
\nabla_{\mathbf{s}^{\prime}} \nabla_{\mathbf{s}^{\prime}}^{\mathrm{T}} F\left(\mathbf{s}^{\circ} ; \mathbf{s}^{\circ}\right)=\left(\begin{array}{ll}
\frac{\partial^{2} F\left(\mathbf{s}^{\prime} ; \mathbf{s}^{\circ}\right)}{\partial x^{\prime 2}} & \frac{\partial^{2} F\left(\mathbf{s}^{\prime} ; \mathbf{s}^{\circ}\right)}{\partial x^{\prime} \partial y^{\prime}} \\
\frac{\partial^{2} F\left(\mathbf{s}^{\prime} ; \mathbf{s}^{\circ}\right)}{\partial x^{\prime} \partial y^{\prime}} & \frac{\partial^{2} F\left(\mathbf{s}^{\prime} ; \mathbf{s}^{\circ}\right)}{\partial y^{\prime 2}}
\end{array}\right)_{\mathbf{s}^{\prime}=\mathbf{s}^{\circ}}=\mathbf{D}\left(\mathbf{s}^{\circ}\right) .
\end{gathered}
$$

When $F\left(\mathbf{s}^{\prime} ; \mathbf{s}^{\circ}\right)$ is regarded as a fitness landscape in the space of mutant trait $\mathbf{s}^{\prime}$ under a fixed resident trait $\mathbf{s}^{\circ}$, Eq. (4a) gives its local gradient, and rescaling of Eq. (4b) gives its local curvature at $\mathbf{s}^{\circ}$ (when $\mathbf{g}\left(\mathbf{s}^{\circ}\right)=\mathbf{0}$, rescaling is not needed; i.e., $\mathbf{v}^{\mathrm{T}} \mathbf{D}\left(\mathbf{s}^{\circ}\right) \mathbf{v}$ gives the curvature along a unit vector $\left.\mathbf{v}\right)$. In this paper, we refer to Eqs. (4a) and (4b) as 'fitness gradient' and 'fitness curvature,' respectively. For convenience, we introduce $\mathbf{g}=\mathbf{g}(\mathbf{s})$ and $\mathbf{D}=\mathbf{D}(\mathbf{s})$. We also introduce another second derivative, $\mathbf{C}$, defined by the first derivative of 


$$
\mathbf{g}\left(\mathbf{s}^{\circ}\right)=\left(\begin{array}{l}
\frac{\partial F\left(\mathbf{s}^{\prime} ; \mathbf{s}^{\circ}\right)}{\partial x^{\prime}} \\
\frac{\partial F\left(\mathbf{s}^{\prime} ; \mathbf{s}^{\circ}\right)}{\partial y^{\prime}}
\end{array}\right)_{\mathbf{s}^{\prime}=\mathbf{s}^{\circ}} \equiv\left(\begin{array}{l}
\frac{\partial F}{\partial x^{\prime}}\left(\mathbf{s}^{\circ} ; \mathbf{s}^{\circ}\right) \\
\frac{\partial F}{\partial y^{\prime}}\left(\mathbf{s}^{\circ} ; \mathbf{s}^{\circ}\right)
\end{array}\right)
$$

at $\mathbf{s}$,

$$
\mathbf{C}^{\mathrm{T}}=\nabla_{\mathbf{s}^{\circ}}^{\mathrm{T}} \mathbf{g}(\mathbf{s})=\left(\begin{array}{ll}
\frac{\partial}{\partial x^{\circ}} \frac{\partial F}{\partial x^{\prime}}\left(\mathbf{s}^{\circ} ; \mathbf{s}^{\circ}\right) & \frac{\partial}{\partial y^{\circ}} \frac{\partial F}{\partial x^{\prime}}\left(\mathbf{s}^{\circ} ; \mathbf{s}^{\circ}\right) \\
\frac{\partial}{\partial x^{\circ}} \frac{\partial F}{\partial y^{\prime}}\left(\mathbf{s}^{\circ} ; \mathbf{s}^{\circ}\right) & \frac{\partial}{\partial y^{\circ}} \frac{\partial F}{\partial y^{\prime}}\left(\mathbf{s}^{\circ} ; \mathbf{s}^{\circ}\right)
\end{array}\right)_{\mathbf{s}^{\prime}=\mathbf{s}^{\circ}=\mathbf{s}}
$$

which describes variability of the fitness gradient at $\mathbf{s}$, depending on $\mathbf{s}^{\circ}$, and thus determines the convergence stability of $\mathbf{s}$ when it is evolutionarily singular. We refer to $\mathbf{C}$ as 'fitness gradient-variability.' Analogous to Eq. (2d), Eq. (4d) is alternatively expressed as

$$
\mathbf{C}=\mathbf{D}+\nabla_{\mathbf{s}^{\circ}} \nabla_{\mathbf{s}^{\prime}}^{\mathrm{T}} F(\mathbf{s} ; \mathbf{s})
$$

where

$$
\nabla_{\mathbf{s}^{\circ}} \nabla_{\mathbf{s}^{\mathrm{T}}}^{\mathrm{T}} F(\mathbf{s} ; \mathbf{s})=\left(\begin{array}{ll}
\frac{\partial}{\partial x^{\prime}} \frac{\partial F}{\partial x^{\circ}}\left(\mathbf{s}^{\circ} ; \mathbf{s}^{\circ}\right) & \frac{\partial}{\partial y^{\prime}} \frac{\partial F}{\partial x^{\circ}}\left(\mathbf{s}^{\circ} ; \mathbf{s}^{\circ}\right) \\
\frac{\partial}{\partial x^{\prime}} \frac{\partial F}{\partial y^{\circ}}\left(\mathbf{s}^{\circ} ; \mathbf{s}^{\circ}\right) & \frac{\partial}{\partial y^{\prime}} \frac{\partial F}{\partial y^{\circ}}\left(\mathbf{s}^{\circ} ; \mathbf{s}^{\circ}\right)
\end{array}\right)_{\mathbf{s}^{\prime}=\mathbf{s}^{\circ}=\mathbf{s}}
$$

\subsection{Lagrange functions for fitness functions in two- dimensional trait spaces}

When no constraint exists, we can directly use $\mathbf{g}$, C, and D to check evolutionary singularity, convergence stability, and evolutionary stability of $\mathbf{s}$, respectively. However, when possible mutants are restricted to the constraint curve $h\left(\mathbf{s}^{\prime}\right)=0$, we need the elements of $\mathbf{g}, \mathbf{C}$, and $\mathbf{D}$ along the curve to check those evolutionarily dynamical properties (Fig. 1). To facilitate such an operation, we integrate the fitness function $F\left(\mathbf{s}^{\prime} ; \mathbf{s}^{\circ}\right)$ and the constraint function $h\left(\mathbf{s}^{\prime}\right)$ into

$$
F_{\mathrm{L}}\left(\mathbf{s}^{\prime} ; \mathbf{s}^{\circ} ; \lambda\right)=F\left(\mathbf{s}^{\prime} ; \mathbf{s}^{\circ}\right)-\lambda\left[h\left(\mathbf{s}^{\prime}\right)-h\left(\mathbf{s}^{\circ}\right)\right],
$$


with a parameter $\lambda$. This function corresponds to the Lagrange function of invasion fitness $F\left(\mathbf{s}^{\prime} ; \mathbf{s}^{\circ}\right)$ with a Lagrange multiplier $\lambda$, called the Lagrange fitness function in this paper. The second term is used to bind the population on the constraint curve. Here, the gradient of Lagrange fitness in $\mathbf{s}^{\prime}$ at $\mathbf{s}$ is

$$
\begin{aligned}
\nabla_{\mathbf{s}^{\prime}} F_{\mathrm{L}}(\mathbf{s} ; \mathbf{s} ; \lambda) & =\nabla_{\mathbf{s}^{\prime}} F(\mathbf{s} ; \mathbf{s})-\lambda \nabla h(\mathbf{s}) \\
= & \mathbf{g}-\lambda|\nabla h(\mathbf{s})| \mathbf{n},
\end{aligned}
$$

where $\mathbf{n}=\left(n_{x}, n_{y}\right)^{\mathrm{T}}=\nabla h(\mathbf{s}) /|\nabla h(\mathbf{s})|$ is the normal vector of the constraint curve at $\mathbf{s}$. Thus, by choosing $\lambda$ at

$$
\lambda_{\mathbf{s}}=\frac{\mathbf{n} \cdot \mathbf{g}}{|\nabla h(\mathbf{s})|}=\frac{\nabla h(\mathbf{s}) \cdot \nabla_{\mathbf{s}^{\prime}} F(\mathbf{s} ; \mathbf{s})}{|\nabla h(\mathbf{s})|^{2}}
$$

where the operator '. ' indicates the inner product of the two vectors, the second term of Eq. (6) becomes the element of $\mathbf{g}$ orthogonal to the curve (i.e., $\lambda \nabla h(\mathbf{s})=$ $[\mathbf{n} \cdot \mathbf{g}] \mathbf{n})$. Consequently, Eq. (6) gives the tangent element of $\mathbf{g}$,

$$
\begin{aligned}
\nabla_{\mathbf{s}^{\prime}} F_{\mathrm{L}}(\mathbf{s} ; \mathbf{s} ; \lambda) & =\mathbf{g}-[\mathbf{n} \cdot \mathbf{g}] \mathbf{n} \\
& =[\mathbf{e} \cdot \mathbf{g}] \mathbf{e}
\end{aligned},
$$

for any $\mathbf{s}$, where $\mathbf{e}=\left(n_{y},-n_{x}\right)^{\mathrm{T}}$ is the tangent vector of the curve at $\mathbf{s}$. Note that the derivative of the second term of Eq. (5) subtracts the orthogonal element $\lambda \nabla h(\mathbf{s})=[\mathbf{n} \cdot \mathbf{g}] \mathbf{n}$ from $\mathbf{g}=[\mathbf{e} \cdot \mathbf{g}] \mathbf{e}+[\mathbf{n} \cdot \mathbf{g}] \mathbf{n}$. Hence, the second term of Eq. (5), $\lambda\left[h\left(\mathbf{s}^{\prime}\right)-h\left(\mathbf{s}^{\circ}\right)\right]$, may be interpreted as a 'harshness' of the constraint on the organism, which removes the possibility of evolution orthogonal to the constraint curve, even if a steep fitness gradient exists in that direction.

\subsection{Conditions for evolutionary branching along constraint curves}

When constraint curves in two-dimensional trait spaces have parametric expressions, the conditions for $\mathbf{s}$ being an evolutionary branching point along the curves are given by Eq. (2). By using the Lagrange fitness function, we can express the left sides of those conditions into ones without parameters:

$$
\begin{gathered}
g(\phi) \mathbf{e}=\nabla_{\mathbf{s}^{\prime}} F_{\mathrm{L}}\left(\mathbf{s} ; \mathbf{s} ; \lambda_{\mathbf{s}}\right), \\
C(\phi)=\mathbf{e}^{\mathrm{T}}\left[\left(\nabla_{\mathbf{s}^{\prime}} \nabla_{\mathbf{s}^{\prime}}^{\mathrm{T}}+\nabla_{\mathbf{s}^{\circ}} \nabla_{\mathbf{s}^{\prime}}^{\mathrm{T}}\right) F_{\mathrm{L}}\left(\mathbf{s} ; \mathbf{s} ; \lambda_{\mathbf{s}}\right)\right] \mathbf{e}=C_{h}, \\
D(\phi)=\mathbf{e}^{\mathrm{T}}\left[\nabla_{\mathbf{s}^{\prime}} \nabla_{\mathbf{s}^{\prime}}^{\mathrm{T}} F_{\mathrm{L}}\left(\mathbf{s} ; \mathbf{s} ; \lambda_{\mathbf{s}}\right)\right] \mathbf{e}=D_{h},
\end{gathered}
$$


where $\left(\nabla_{\mathbf{s}^{\prime}} \nabla_{\mathbf{s}^{\prime}}^{\mathrm{T}}+\nabla_{\mathbf{s}^{\circ}} \nabla_{\mathbf{s}^{\prime}}^{\mathrm{T}}\right) F_{\mathrm{L}}\left(\mathbf{s} ; \mathbf{s} ; \lambda_{\mathbf{s}}\right)=\nabla_{\mathbf{s}^{\prime}} \nabla_{\mathbf{s}^{\prime}}^{\mathrm{T}} F_{\mathrm{L}}\left(\mathbf{s} ; \mathbf{s} ; \lambda_{\mathbf{s}}\right)+\nabla_{\mathbf{s}^{\circ}} \nabla_{\mathbf{s}^{\prime}}^{\mathrm{T}} F_{\mathrm{L}}\left(\mathbf{s} ; \mathbf{s} ; \lambda_{\mathbf{s}}\right)$, and appropriate scaling of $\phi$ is assumed so that $\left|\left(\frac{\mathrm{d} x(\phi)}{\mathrm{d} \phi}, \frac{\mathrm{d} y(\phi)}{\mathrm{d} \phi}\right)\right|=1$ without loss of generality (Appendix A.3). Moreover, we have the following theorem for an arbitrary constraint curve described with $h\left(\mathbf{s}^{\prime}\right)=0$ (see Appendix A.1-2 for the proof).

\section{Theorem 1: Branching conditions along constraint (two- dimensional trait spaces)}

In two-dimensional trait space $\boldsymbol{s}=(x, y)^{T}$, a point $\boldsymbol{s}$ is an evolutionary branching point along the constraint curve $h(\boldsymbol{s})=0$, if $\boldsymbol{s}$ satisfies the following three conditions of the Lagrange fitness function Eq. (5) with Eq. (7):

(i) $\boldsymbol{s}$ is evolutionarily singular along the constraint curve $h(\boldsymbol{s})=0$, satisfying

$$
\nabla_{\mathbf{s}^{\prime}} F_{\mathrm{L}}\left(\mathbf{s} ; \mathbf{s} ; \lambda_{\mathbf{s}}\right)=\mathbf{0} .
$$

(ii) $\boldsymbol{s}$ is convergence stable along the constraint curve, satisfying

$$
C_{\mathrm{h}}=\mathbf{e}^{\mathrm{T}}\left[\left(\nabla_{\mathbf{s}^{\prime}} \nabla_{\mathbf{s}^{\prime}}^{\mathrm{T}}+\nabla_{\mathbf{s}^{\circ}} \nabla_{\mathbf{s}^{\prime}}^{\mathrm{T}}\right) F_{\mathrm{L}}\left(\mathbf{s} ; \mathbf{s} ; \lambda_{\mathbf{s}}\right)\right] \mathbf{e}<0 .
$$

(iii) $\boldsymbol{s}$ is evolutionarily unstable along the constraint curve, satisfying

$$
D_{\mathrm{h}}=\mathbf{e}^{\mathrm{T}}\left[\nabla_{\mathbf{s}^{\prime}} \nabla_{\mathbf{s}^{\prime}}^{\mathrm{T}} F_{\mathrm{L}}\left(\mathbf{s} ; \mathbf{s} ; \lambda_{\mathbf{s}}\right)\right] \mathbf{e}>0 .
$$

By Eq. (8), we can transform Eq. (10a) into

$$
\mathbf{e} \cdot \nabla_{\mathbf{s}^{\prime}} F(\mathbf{s} ; \mathbf{s})=0,
$$

which may be easier to check. Table 1 summarizes how the fitness gradient, gradient variability, and curvature along the constraint curve are expressed in terms of the Lagrange fitness function.

\subsection{Relationship with standard Lagrange multiplier method}

Since $\lambda=\lambda_{\mathbf{s}}$, defined by Eq. (7), can also be derived as the solution of condition (i), $\lambda$ can be left as an unknown parameter satisfying condition (i), like a Lagrange multiplier in the standard Lagrange multiplier method. In this case, conditions (i) and (iii) are equivalent to the conditions for stationary points and local minima ('second derivative test') in the standard method. When the fitness function is independent of resident phenotypes, $C_{\mathrm{h}}=D_{\mathrm{h}}$ always holds. In this case, condition (ii) $C_{\mathrm{h}}<0$ is never satisfied when condition (iii) $D_{\mathrm{h}}>0$ holds. However, when the fitness function depends on resident phenotypes (i.e., frequency-dependent 
fitness functions), satisfying condition (ii) is decoupled from not satisfying condition (iii). Thus, Theorem 1 is a modification of the standard Lagrange multiplier method to analyze frequency-dependent fitness functions by adding condition (ii) for convergence stability. In the standard method, $D_{\mathrm{h}}$ can be examined with the corresponding bordered Hessian matrix (Eq. (22b)). Analogous calculations can be used to examine $C_{\mathrm{h}}$ (Eq. (22d)).

The above relationships hold also for the higher-dimensional constraint surfaces explained in the next section. Like the standard method, our method is completely analytical.

\subsection{Effect of constraint curve curvature}

Here, we explain how the curvature of the constraint curve affects the conditions for evolutionary branching (Eq. (10)). The curvature does not affect evolutionary singularity because Eq. (10a) is equivalent to Eq. (11), since it does not contain second derivatives of the constraint. On the other hand, convergence stability and evolutionary stability are both affected by the curvature, as previous studies have shown graphically (Rueffler et al. 2004; deMazancourt and Dieckmann, 2004) and analytically with parameterization (Appendix A in deMazancourt and Dieckmann, 2004; Kisdi, 2006). This feature is shown more clearly in our method without parameterization by transforming the left sides of Eqs. (10b) and (10c) into

$$
\begin{aligned}
C_{\mathrm{h}} & =\mathbf{e}^{\mathrm{T}}\left[\left(\nabla_{\mathbf{s}^{\prime}} \nabla_{\mathbf{s}^{\prime}}^{\mathrm{T}}+\nabla_{\mathbf{s}^{\circ}} \nabla_{\mathbf{s}^{\prime}}^{\mathrm{T}}\right) F(\mathbf{s} ; \mathbf{s})\right] \mathbf{e}-\mathbf{e}^{\mathrm{T}}\left[\lambda_{\mathbf{s}} \nabla \nabla^{\mathrm{T}} h(\mathbf{s})\right] \mathbf{e} \\
& =\mathbf{e}^{\mathrm{T}} \mathbf{C e}+\Omega, \\
D_{\mathrm{h}} & =\mathbf{e}^{\mathrm{T}}\left[\nabla_{\mathbf{s}^{\prime}} \nabla_{\mathbf{s}^{\prime}}^{\mathrm{T}} F(\mathbf{s} ; \mathbf{s})\right] \mathbf{e}-\mathbf{e}^{\mathrm{T}}\left[\lambda_{\mathbf{s}} \nabla \nabla^{\mathrm{T}} h(\mathbf{s})\right] \mathbf{e} \\
& =\mathbf{e}^{\mathrm{T}} \mathbf{D e}+\Omega,
\end{aligned}
$$

where, noting Eq. (7),

$$
\begin{aligned}
\Omega & =-\mathbf{e}^{\mathrm{T}}\left[\lambda_{\mathbf{s}} \nabla \nabla^{\mathrm{T}} h(\mathbf{s})\right] \mathbf{e} \\
& =\mathbf{g} \cdot\left[-\frac{\mathbf{e}^{\mathrm{T}} \nabla \nabla^{\mathrm{T}} h(\mathbf{s}) \mathbf{e}}{|\nabla h(\mathbf{s})|} \mathbf{n}\right]=\mathbf{g} \cdot \mathbf{q} .
\end{aligned}
$$

The first terms in Eq. (12a), $\mathbf{e}^{\mathrm{T}} \mathbf{C e}$ and $\mathbf{e}^{\mathrm{T}} \mathbf{D e}$, give fitness gradient variability and fitness curvature, respectively, for $\mathbf{s}$ along the curve when the constraint curve is a straight line. The effect of the constraint curvature is given by $\Omega$, which is the 
inner product of the fitness gradient $\mathbf{g}$ and a curvature vector $\mathbf{q}$ at $\mathbf{s}$. The curvature vector is a scaled normal vector

$$
\mathbf{q}=q \mathbf{n}
$$

with

$$
q=-\frac{\mathbf{e}^{\mathrm{T}} \nabla \nabla^{\mathrm{T}} h(\mathbf{s}) \mathbf{e}}{|\nabla h(\mathbf{s})|},
$$

so that its length $|q|$ is equal to the reciprocal of the curvature radius. Specifically, the constraint curve $h\left(\mathbf{s}^{\prime}\right)=0$ can be described locally with

$$
\tilde{y}^{\prime}=\frac{1}{2} q\left(\tilde{x}^{\prime 2}\right)+\mathrm{O}\left(\tilde{x}^{\prime 3}\right)
$$

with the $\tilde{x}$-and $\tilde{y}$-axes given by $\mathbf{e}$ and $\mathbf{n}$, i.e., $\tilde{x}^{\prime}=\mathbf{e} \cdot\left(\mathbf{s}^{\prime}-\mathbf{s}\right)$ and $\tilde{y}^{\prime}=\mathbf{n} \cdot$ $\left(\mathbf{s}^{\prime}-\mathbf{s}\right)$ (Fig. 2a).

Note that $C_{\mathrm{h}}$ and $D_{\mathrm{h}}$ in Eq. (12a) have the same second term $\Omega=\mathbf{g} \cdot \mathbf{q}$. Thus, the effects of the curvature on $C_{\mathrm{h}}$ and $D_{\mathrm{h}}$ are large when the element of the fitness gradient orthogonal to the curve is large, as illustrated in Figure 2. If their directions, i.e., those of the fitness gradient and curvature vector, are opposite, the resulting negative curvature effect $\Omega$ decreases both $C_{\mathrm{h}}$ and $D_{\mathrm{h}}$ (Fig. 2a), which makes the point $\mathbf{s}$ more convergence and evolutionarily stable (Fig. 2c). Conversely, if they have the same direction, the resulting positive curvature effect increases both $C_{\mathrm{h}}$ and $D_{\mathrm{h}}$ (Fig. $2 \mathrm{~b}$ ), which makes the point $\mathbf{s}$ less convergence and evolutionarily stable (Fig. $2 \mathrm{~d}$ ). When $\Omega$ results in negative $C_{\mathrm{h}}$ and positive $D_{\mathrm{h}}$ simultaneously, $\mathbf{s}$ is an evolutionary branching point along the constraint curve. Note that even when the original two-dimensional fitness landscape is flat, i.e., $\mathbf{D}=\mathbf{0}$, the fitness landscape along the constraint curve has a curvature $D_{\mathrm{h}}=$ $\Omega$ when $\Omega \neq 0$. In this sense, we refer to $\Omega$ as apparent fitness curvature.

\section{Extension to higher dimensionalities}

In this section, we extend the two-dimensional method discussed above for higher dimensionalities. We consider an arbitrary $M$-dimensional trait space $\mathbf{s}=$ $\left(x_{1}, \ldots, x_{M}\right)^{\mathrm{T}}$ and an invasion fitness function $F\left(\mathbf{s}^{\prime} ; \mathbf{s}^{\circ}\right)$. For an arbitrary position $\mathbf{s}$, the fitness gradient, fitness gradient variability, and fitness curvature are written in the same manner as the two-dimensional case: 


$$
\begin{aligned}
& \mathbf{g}=\nabla_{\mathbf{s}^{\prime}} F(\mathbf{s} ; \mathbf{s}), \\
& \mathbf{C}=\nabla_{\mathbf{s}^{\prime}} \nabla_{\mathbf{s}^{\prime}}^{\mathrm{T}} F(\mathbf{s} ; \mathbf{s})+\nabla_{\mathbf{s}} \nabla_{\mathbf{s}^{\prime}}^{\mathrm{T}} F(\mathbf{s} ; \mathbf{s})=\left(\nabla_{\mathbf{s}^{\prime}} \nabla_{\mathbf{s}^{\prime}}^{\mathrm{T}}+\nabla_{\mathbf{s}^{\circ}} \nabla_{\mathbf{s}^{\prime}}^{\mathrm{T}}\right) F(\mathbf{s} ; \mathbf{s}), \\
& \mathbf{D}=\nabla_{\mathbf{s}^{\prime}} \nabla_{\mathbf{s}^{\prime}}^{\mathrm{T}} F(\mathbf{s} ; \mathbf{s}) .
\end{aligned}
$$

We consider an arbitrary $L$-dimensional constraint surface defined by $h_{j}\left(\mathbf{s}^{\prime}\right)=0$ for $j=L+1, \cdots, M$, to which all possible mutants $\mathbf{s}^{\prime}$ are restricted. To analyze adaptive evolution along the constraint surface, we obtain the elements $\mathbf{g}$, $\mathbf{C}$, and D along the surface as follows.

\subsection{Lagrange fitness function for constraint surface}

As described in Lemma 2 in Appendix C, the Lagrange fitness function for the constraint surface is constructed as

$$
F_{\mathrm{L}}\left(\mathbf{s}^{\prime} ; \mathbf{s}^{\circ} ; \lambda\right)=F\left(\mathbf{s}^{\prime} ; \mathbf{s}^{\circ}\right)-\sum_{j=L+1}^{M} \lambda_{j}\left[h_{j}\left(\mathbf{s}^{\prime}\right)-h_{j}\left(\mathbf{s}^{\circ}\right)\right],
$$

with $\lambda=\left(\lambda_{L+1}, \ldots, \lambda_{M}\right)^{\mathrm{T}}$. When the normal vectors $\mathbf{n}_{j}=\nabla h_{j}(\mathbf{s}) /\left|\nabla h_{j}(\mathbf{s})\right|$ for $j=L+1, \cdots, M$ are orthogonal, we can choose

$$
\lambda_{\mathbf{s} j}=\frac{\mathbf{n}_{j} \cdot \mathbf{g}}{\left|\nabla h_{j}(\mathbf{s})\right|},
$$

such that the gradient of the second term of Eq. (15) with respect to $\mathbf{s}^{\prime}$ gives the element of $\mathbf{g}$ orthogonal to the surface,

$$
\sum_{j=L+1}^{M} \lambda_{\mathbf{s} j} \nabla h_{j}(\mathbf{s})=\sum_{j=L+1}^{M}\left[\mathbf{n}_{j} \cdot \mathbf{g}\right] \mathbf{n}_{j}
$$

Thus, the gradient of Eq. (15) gives the tangent element of $\mathbf{g}$,

$$
\begin{aligned}
\nabla_{\mathbf{s}^{\prime}} F_{\mathrm{L}}\left(\mathbf{s} ; \mathbf{s} ; \boldsymbol{\lambda}_{\mathbf{s}}\right) & =-\sum_{j=L+1}^{M}\left[\mathbf{n}_{j} \cdot \mathbf{g}\right] \mathbf{n}_{j} \\
& =\sum_{i=1}^{L}\left[\mathbf{e}_{i} \cdot \mathbf{g}\right] \mathbf{e}_{i}=\mathbf{E} \mathbf{E}^{\mathrm{T}} \mathbf{g},
\end{aligned}
$$

where $\mathbf{E}=\left(\mathbf{e}_{1}, \cdots, \mathbf{e}_{L}\right)$ are the tangent vectors of unit lengths, which are chosen to be orthogonal (e.g., with Gram-Schmidt orthonormalization) without losing generality. 
Even in general cases where the normal vectors may not be orthogonal, we can make $\nabla_{\mathbf{s}^{\prime}} F_{\mathrm{L}}\left(\mathbf{s} ; \mathbf{s} ; \boldsymbol{\lambda}_{\mathbf{s}}\right)=\sum_{i=1}^{L}\left[\mathbf{e}_{i} \cdot \mathbf{g}\right] \mathbf{e}_{i}$ hold [Eq. (C.15) in Appendix C] by choosing $\lambda$ at

$$
\lambda_{\mathbf{s}}=\mathbf{B}^{+} \nabla_{\mathbf{s}^{\prime}} F(\mathbf{s} ; \mathbf{s})=\mathbf{B}^{+} \mathbf{g},
$$

where $\mathbf{B}^{+}=\left[\mathbf{B}^{\mathrm{T}} \mathbf{B}\right]^{-1} \mathbf{B}^{\mathrm{T}}$ is the pseudo inverse of $\mathbf{B}=\left(\nabla h_{L+1}(\mathbf{s}), \cdots, \nabla h_{M}(\mathbf{s})\right)$, i.e., $\mathbf{B}^{+} \mathbf{B}$ gives the $(M-L)$-dimensional identity matrix $\mathbf{I}_{M-L}$. In statistics, $\boldsymbol{\lambda}_{\boldsymbol{s}}$ is the regression coefficients for predictor variables $\nabla h_{L+1}(\mathbf{s}), \cdots, \nabla h_{M}(\mathbf{s})$, to explain $\mathbf{g}$. When the normal vectors are orthogonal, Eq. (19) yields Eq. (16).

\subsection{Conditions for the existence of CBPs along constraint surfaces}

The dimensionalities of constraint surfaces can be greater than one, in which case one-dimensional conditions for evolutionary branching cannot be applied. As for multi-dimensional conditions for evolutionary branching, numerical simulations of adaptive evolution in various eco-evolutionary settings (Vukics et al., 2003; Ackermann and Doebeli, 2004; Egas et al., 2005; Ito and Dieckmann, 2012) have shown that evolutionary branching arises in the neighborhood of a point $\mathbf{s}$, if $\mathbf{s}$ is (i) evolutionarily singular, (ii) strongly convergence stable (Leimar, 2005, 2009), and (iii) evolutionarily unstable. Among these three conditions, conditions (i) and (iii) are simply extensions of conditions (i) and (iii) in the one-dimensional case [Eq. (2)], respectively. Condition (i) means the disappearance of the fitness gradient for the resident located at $\mathbf{s}$, and condition (iii) means that the fitness landscape is concave along at least one direction. On the other hand, condition (ii) introduces the new term 'strongly convergence stable,' which means convergence stability under any genetic correlation in the multi-dimensional mutant phenotype (see Leimar, 2005 for the proof of strong convergence stability).

Currently, no formal proof has determined whether the existence of points satisfying (i-iii) is sufficient for evolutionary branching to occur in the neighborhood of those points, although substantial progress has been made (see section 7). In this paper, we refer to points satisfying (i-iii) as CBPs (candidate branching points). By applying the three conditions for CBPs, we establish the following multi-dimensional conditions for CBPs along the constraint surface (see Appendix C for the proof). 


\section{Theorem 2: Conditions for existence of CBPs along constraints (multi-dimensional)}

In an arbitrary $M$-dimensional trait space $\mathbf{s}=\left(x_{1}, \cdots, x_{M}\right)^{\mathrm{T}}$, a point $\mathbf{s}$ is a CBP (i.e., a point that is strongly convergence and evolutionarily unstable) along an arbitrary L-dimensional constraint surface defined by $h_{j}(\mathbf{s})=0$ for $j=L+1, \cdots, M$, if $\mathbf{s}$ satisfies the following three conditions of the Lagrange fitness function Eq. (15) with Eq. (19):

(i) $\mathbf{s}$ is evolutionarily singular along the constraint surface, satisfying

$$
\nabla_{\mathbf{s}^{\prime}} F_{\mathrm{L}}\left(\mathbf{s} ; \mathbf{s} ; \boldsymbol{\lambda}_{\mathbf{s}}\right)=\mathbf{0} .
$$

(ii) $\mathbf{s}$ is strongly convergence stable along the constraint surface, i.e., the symmetric part of an L-by-L matrix

$$
\mathbf{C}_{\mathrm{h}}=\mathbf{E}^{\mathrm{T}}\left[\left(\nabla_{\mathbf{s}^{\prime}} \nabla_{\mathbf{s}^{\prime}}^{\mathrm{T}}+\nabla_{\mathbf{s}^{\circ}} \nabla_{\mathbf{s}^{\prime}}^{\mathrm{T}}\right) F_{\mathrm{L}}\left(\mathbf{s} ; \mathbf{s} ; \boldsymbol{\lambda}_{\mathbf{s}}\right)\right] \mathbf{E}
$$

is negative definite, where an $M$-by-L matrix $\mathbf{E}=\left(\mathbf{e}_{1}, \cdots, \mathbf{e}_{L}\right)$ consists of orthogonal base vectors $\mathbf{e}_{1}, \ldots, \mathbf{e}_{L}$ of the tangent plane of the constraint surface at $\mathbf{s .}$

(iii) $\mathbf{s}$ is evolutionarily unstable along the constraint surface, i.e., a symmetric L-by-L matrix

$$
\mathbf{D}_{\mathrm{h}}=\mathbf{E}^{\mathrm{T}} \nabla_{\mathbf{s}^{\prime}} \nabla_{\mathbf{s}^{\prime}}^{\mathrm{T}} F_{\mathrm{L}}\left(\mathbf{s} ; \mathbf{s} ; \boldsymbol{\lambda}_{\mathbf{s}}\right) \mathbf{E}
$$

has at least one positive eigenvalue.

Analogous to the two-dimensional case, we can transform Eq. (20a) using Eq. (18) into

$$
\mathbf{E}^{\mathrm{T}} \nabla_{\mathbf{s}^{\prime}} F(\mathbf{s} ; \mathbf{s})=\mathbf{0} .
$$

Table 2 summarizes how the fitness gradient, gradient variability, and curvature along the constraint surface are expressed in terms of the Lagrange fitness function.

\subsection{Bordered second-derivative matrix}

In the standard Lagrange multiplier method, whether an extremum is maximum, minimum, or saddled along the constraint surface can be examined with the corresponding bordered Hessian matrix (Mandy, 2013), in which calculation of $\mathbf{E}$, the base vectors of the tangent plane, is not needed. This technique is also useful for examining not only $\mathbf{D}_{h}$, but also $\mathbf{C}_{h}$, as explained below. For convenience, we denote the number of equality constraints by $N=M-L$. In this paper, we define the bordered Hessian for $\mathbf{D}_{\mathrm{h}}$ by a square matrix with size $N+M$,

$$
\mathbf{D}_{\mathrm{B}}=\left(\begin{array}{cc}
\mathbf{0} & \mathbf{B}^{\mathrm{T}} \\
\mathbf{B} & -\mathbf{D}_{\mathrm{L}}
\end{array}\right),
$$


where $\mathbf{B}=\left(\nabla h_{L+1}(\mathbf{s}), \cdots, \nabla h_{M}(\mathbf{s})\right), \mathbf{D}_{\mathrm{L}}=\nabla_{\mathbf{s}^{\prime}} \nabla_{\mathbf{s}^{\prime}}^{\mathrm{T}} F_{\mathrm{L}}\left(\mathbf{s} ; \mathbf{s} ; \boldsymbol{\lambda}_{\mathbf{s}}\right)$, and trait axes are permutated appropriately so that separation of $\mathbf{s}=\left(x_{1}, \cdots, x_{M}\right)^{\mathrm{T}}$ into $\mathbf{x}=$ $\left(x_{1}, \cdots, x_{L}\right)^{\mathrm{T}}$ and $\mathbf{y}=\left(x_{L+1}, \cdots, x_{M}\right)^{\mathrm{T}}$ makes an $N \times N$ matrix $\left(\nabla_{\mathbf{y}^{\prime}} h_{L+1}(\mathbf{s}), \cdots, \nabla_{\mathbf{y}^{\prime}} h_{M}(\mathbf{s})\right)$ nonsingular. Note that $\mathbf{D}_{\mathrm{L}}$ is multiplied by -1 , which differentiates it slightly from the standard bordered Hessian, but simplifies the analysis of evolutionary stability along the surface (i.e., negative definiteness of $\left.\mathbf{D}_{\mathrm{h}}=\mathbf{E}^{\mathrm{T}} \mathbf{D}_{\mathrm{L}} \mathbf{E}\right)$. Similarly, to analyze strong convergence stability along the surface, we define a bordered second-derivative matrix

$$
\mathbf{C}_{\mathrm{B}}=\left(\begin{array}{cc}
\mathbf{0} & \mathbf{B}^{\mathrm{T}} \\
\mathbf{B} & -1 / 2\left(\mathbf{C}_{\mathrm{L}}+\mathbf{C}_{\mathrm{L}}^{\mathrm{T}}\right)
\end{array}\right),
$$

where $\mathbf{C}_{\mathrm{L}}=\left(\nabla_{\mathbf{s}^{\prime}} \nabla_{\mathbf{s}^{\prime}}^{\mathrm{T}}+\nabla_{\mathbf{s}^{\circ}} \nabla_{\mathbf{s}^{\prime}}^{\mathrm{T}}\right) F_{\mathrm{L}}\left(\mathbf{s} ; \mathbf{s} ; \boldsymbol{\lambda}_{\mathbf{s}}\right)$. Then, we have the following two corollaries (see Appendix E for the derivation).

\section{Corollary 1: Evolutionary stability condition by bordered Hessian}

A point $\boldsymbol{s}$ satisfying Eq. (20a) is locally evolutionarily stable along the constraint surface described in Theorem 2 (i.e., $\boldsymbol{D}_{h}$ is negative definite) if every principal minor of $\boldsymbol{D}_{B}$ of order $k=2 N+1, \cdots, N+M$ has the sign $(-1)^{N}$, where $N=M-L$, and the $k$ th principal minor of $\boldsymbol{D}_{B}$ is given by the determinant of the upper left $k \times k$ submatrix of $\boldsymbol{D}_{B}$,

$$
\left|\mathbf{D}_{\mathrm{B}}^{(k)}\right|=\left|\begin{array}{ccc}
\mathbf{D}_{\mathrm{B}, 11} & \cdots & \mathbf{D}_{\mathrm{B}, k 1} \\
\vdots & \ddots & \vdots \\
\mathbf{D}_{\mathrm{B}, 1 k} & \cdots & \mathbf{D}_{\mathrm{B}, k k}
\end{array}\right| .
$$

Conversely, $\boldsymbol{s}$ is evolutionarily unstable along the constraint surface (i.e., $\boldsymbol{D}_{h}$ has at least one positive eigenvalue) if Eq. (22a) for either of $k=2 \mathrm{~N}+$ $1, \cdots, N+M$ has a sign other than $(-1)^{N}$. For one-dimensional constraint curves in two-dimensional trait spaces $(L=1, M=2)$,

$$
D_{\mathrm{h}}=\frac{\left|\mathbf{D}_{\mathrm{B}}\right|}{|\nabla h(\mathbf{s})|^{2}} \text {. }
$$

\section{Corollary 2: Strong convergence stability condition by bordered second-derivative matrix}

A point $\boldsymbol{s}$ satisfying Eq. (20a) is strongly convergence stable along the constraint surface described in Theorem 2 (i.e., $C_{h}$ has a negative definite symmetric part) if every principal minor of $\boldsymbol{C}_{B}$ of order $k=2 \mathrm{~N}+$ $1, \cdots, N+M$ has the sign $(-1)^{N}$, where $N=M-L$, and the $k$ th principal minor of $C_{B}$ is given by 


$$
\left|\mathbf{C}_{\mathrm{B}}^{(k)}\right|=\left|\begin{array}{ccc}
\mathbf{C}_{\mathrm{B}, 11} & \cdots & \mathbf{C}_{\mathrm{B}, k 1} \\
\vdots & \ddots & \vdots \\
\mathbf{C}_{\mathrm{B}, 1 k} & \cdots & \mathbf{C}_{\mathrm{B}, k k}
\end{array}\right|
$$

For one-dimensional constraint curves in two-dimensional trait spaces $(L=$ $1, M=2)$,

$$
C_{\mathrm{h}}=\frac{\left|\mathbf{C}_{\mathrm{B}}\right|}{|\nabla h(\mathbf{s})|^{2}}
$$

\subsection{Effect of constraint surface curvature}

The fitness landscape along the constraint surface is affected by the curvature of the surface, similar to the two-dimensional case. For example, if the surface curves along a tangent vector $\mathbf{e}_{i}$ in the direction of original fitness gradient $\mathbf{g}$, as in Fig. $2 \mathrm{~b}$ with $\mathbf{e}_{i}=\mathbf{e}\left(\tilde{x}\right.$-axis), the curvature makes the fitness landscape along $\mathbf{e}_{i}$ more concave, as in Fig. 2d. Specifically, Eqs. (20b) and (20c) are transformed to

$$
\begin{aligned}
\mathbf{C}_{\mathrm{h}} & =\mathbf{E}^{\mathrm{T}}\left[\left(\nabla_{\mathbf{s}^{\prime}} \nabla_{\mathbf{s}^{\prime}}^{\mathrm{T}}+\nabla_{\mathbf{s}^{\circ}} \nabla_{\mathbf{s}^{\prime}}^{\mathrm{T}}\right) F(\mathbf{s} ; \mathbf{s})\right] \mathbf{E}-\sum_{j=L+1}^{M} \lambda_{\mathbf{s} j} \mathbf{E}^{\mathrm{T}} \nabla \nabla^{\mathrm{T}} \mathrm{h}_{j}(\mathbf{s}) \mathbf{E} \\
& =\mathbf{E}^{\mathrm{T}} \mathbf{C E}+\mathbf{\Omega}, \\
\mathbf{D}_{\mathrm{h}} & =\mathbf{E}^{\mathrm{T}}\left[\nabla_{\mathbf{s}^{\prime}} \nabla_{\mathbf{s}^{\prime}}^{\mathrm{T}} F(\mathbf{s} ; \mathbf{s})\right] \mathbf{E}-\sum_{j=L+1}^{M} \lambda_{\mathbf{s} j} \mathbf{E}^{\mathrm{T}} \nabla \nabla^{\mathrm{T}} h_{j}(\mathbf{s}) \mathbf{E} \\
& =\mathbf{E}^{\mathrm{T}} \mathbf{D E}+\mathbf{\Omega},
\end{aligned}
$$

where the first terms in Eq. (23a), $\mathbf{E}^{\mathrm{T}} \mathbf{C E}$ and $\mathbf{E}^{\mathrm{T}} \mathbf{D E}$, give fitness gradient variability and fitness curvature, respectively, for $\mathbf{S}$ along the surface when the surface is locally flat. The effect of the constraint curvature, i.e., apparent fitness curvature, is given by an $L$-by- $L$ matrix

$$
\boldsymbol{\Omega}=-\sum_{j=L+1}^{M} \lambda_{\mathbf{s} j} \mathbf{E}^{\mathrm{T}} \nabla \nabla^{\mathrm{T}} h_{j}(\mathbf{s}) \mathbf{E} .
$$

This effect can be expressed as a kind of inner product of the fitness gradient and local curvature of the constraint surface, analogous to the two-dimensional case (Eq. (12b); Appendix F). 


\section{Potential for evolutionary branching}

The method described in the above sections is used to find CBPs under given constraint surfaces. In this section, we consider cases in which we can freely choose dimensions and shapes. With this freedom, we can adjust $\mathbf{C}_{h}$ and $\mathbf{D}_{h}$ in Eq. (23a) using the apparent fitness curvature $\boldsymbol{\Omega}$, such that the point $\mathbf{s}$ becomes a CBP. By applying this operation to all points in a trait space, we can examine whether the trait space has CBPs by choosing an appropriate constraint surface. This type of analysis was originally developed for one-dimensional constraint curves in two-dimensional trait spaces using graphical approaches (Bowers et al. 2003, 2005; Rueffler et al. 2004; de Mazancourt and Dieckmann 2004) and analytical approaches with parameterization (de Mazancourt and Dieckmann 2004; Kisdi 2006; Geritz et al. 2007). The latter approach has been extended further for one-dimensional constraint curves in trait spaces of arbitrary dimensions (Kisdi 2015). Here, we extend this analysis for constraint surfaces with arbitrary dimensions by using Theorem 2 from above.

The basic idea is as follows. For an arbitrary point $\mathbf{s}$, we first adjust $\boldsymbol{\Omega}$ in Eq. (23a) so that the symmetric part of $\mathbf{C}_{\mathrm{h}}$ becomes a zero matrix (i.e., neutrally convergence stable). If the largest eigenvalue of $\mathbf{D}_{\mathrm{h}}$ is still positive (evolutionarily unstable), then we can slightly adjust $\boldsymbol{\Omega}$ so that the symmetric part of $\mathbf{C}_{\mathrm{h}}$ becomes slightly negative definite (strongly convergence stable) while the largest eigenvalue of $\mathbf{D}_{\mathrm{h}}$ remains positive. This operation is possible whenever $\mathbf{v}^{\mathrm{T}}(\mathbf{D}-\mathbf{C}) \mathbf{v}>0$ holds for some vector $\mathbf{v}$ orthogonal to the fitness gradient $\mathbf{g}$. More specifically, we have the following theorem (see Appendix G for the proof).

\section{Theorem 3: Potential for evolutionary branching}

For a fitness function $F\left(\mathbf{s}^{\prime} ; \mathbf{s}^{\circ}\right)$ defined on an arbitrary $M$-dimensional trait space $\mathbf{s}=\left(x_{1}, \cdots, x_{M}\right)^{\mathrm{T}}$, if a point $\mathbf{s}$ satisfies the branching potential condition: the symmetric $M-b y-M$ matrix

$$
\mathbf{P}=\frac{1}{2} \mathbf{U}^{\mathrm{T}}\left[\mathbf{M}+\mathbf{M}^{\mathrm{T}}\right] \mathbf{U}
$$

has at least one positive eigenvalue, then $\boldsymbol{s}$ is a CBP (a point that is strongly convergence stable and evolutionarily unstable) along an $(M-1)$ dimensional constraint surface, given by

$$
\begin{aligned}
h\left(\mathbf{s}^{\prime}\right)= & \mathbf{g}^{\mathrm{T}}\left[\mathbf{s}^{\prime}-\mathbf{s}\right] \\
& +\frac{1}{2}\left[\mathbf{s}^{\prime}-\mathbf{s}\right]^{\mathrm{T}}\left[\frac{1}{2}\left(\mathbf{C}+\mathbf{C}^{\mathrm{T}}\right)+\widetilde{\varepsilon} \mathbf{I}\right]\left[\mathbf{s}^{\prime}-\mathbf{s}\right]+\mathrm{O}\left(\left|\mathbf{s}^{\prime}-\mathbf{s}\right|^{3}\right)=0,
\end{aligned}
$$

with a positive $\tilde{\varepsilon}$ that is smaller than the maximum eigenvalue of $\boldsymbol{P}$, where 


$$
\begin{aligned}
& \mathbf{U}=\mathbf{I}-\frac{\mathbf{g g}^{\mathrm{T}}}{|\mathbf{g}|^{2}} \\
& \mathbf{M}=\mathbf{D}-\mathbf{C}=-\nabla_{\mathbf{s}^{\circ}} \nabla_{\mathbf{s}^{\prime}}^{\mathrm{T}} F(\mathbf{s} ; \mathbf{s}), \\
& \mathbf{g}=\nabla_{\mathbf{s}^{\prime}} F(\mathbf{s} ; \mathbf{s}), \\
& \mathbf{C}=\nabla_{\mathbf{s}^{\prime}} \nabla_{\mathbf{s}^{\prime}}^{\mathrm{T}} F(\mathbf{s} ; \mathbf{s})+\nabla_{\mathbf{s}^{\circ}} \nabla_{\mathbf{s}^{\prime}}^{\mathrm{T}} F(\mathbf{s} ; \mathbf{s}) .
\end{aligned}
$$

The dimensionality of the constraint surface can be reduced arbitrarily by adding appropriate equality constraints.

In this paper, we refer to the matrix $\mathbf{P}$ as the 'branching potential matrix.' The branching potential condition is also expressed as $\mathbf{v}^{\mathrm{T}} \mathbf{M v}>0$ for some vector $\mathbf{v}$ orthogonal to $\mathbf{g}$ (because $\mathbf{v}^{\mathrm{T}} \mathbf{M v}>0$ gives $\mathbf{v}^{\mathrm{T}} \mathbf{P v}>0$, which is sufficient for $\mathbf{P}$ to have at least one positive eigenvalue). This ensures the coexistence of two slightly different phenotypes in the neighborhood of $\mathbf{s}$, i.e., $F\left(\mathbf{s}_{1} ; \mathbf{s}_{2}\right)>0$ and $F\left(\mathbf{s}_{2} ; \mathbf{s}_{1}\right)>0$ for $\mathbf{s}_{1}=\mathbf{s}+\varepsilon \mathbf{v}$ and $\mathbf{s}_{2}=\mathbf{s}-\varepsilon \mathbf{v}$ for positive and sufficiently small $\varepsilon$.

Analogous to Corollaries 1 and 2 in the previous section, we can translate Theorem 3 to one based on a bordered second-derivative matrix

$$
\mathbf{M}_{\mathrm{B}}=\left(\begin{array}{cc}
\mathbf{0} & \mathbf{g}^{\mathrm{T}} \\
\mathbf{g} & -1 / 2\left(\mathbf{M}_{\mathrm{L}}+\mathbf{M}_{\mathrm{L}}^{\mathrm{T}}\right)
\end{array}\right),
$$

with $\mathbf{M}_{\mathrm{L}}=-\nabla_{\mathbf{s}^{\circ}} \nabla_{\mathbf{s}^{\prime}}^{\mathrm{T}} F_{\mathrm{L}}(\mathbf{s} ; \mathbf{s})=-\nabla_{\mathbf{s}^{\circ}} \nabla_{\mathbf{s}^{\prime}}^{\mathrm{T}} F(\mathbf{s} ; \mathbf{s})=\mathbf{M}$, as follows.

\section{Corollary 3: Branching potential condition by bordered second- derivative matrix}

A point $\boldsymbol{s}$ is a CBP (a point that is strongly convergence stable and evolutionarily unstable) along an $(M-1)$-dimensional constraint surface, given by Eq. (24b), if either principal minor of $\boldsymbol{M}_{B}$

$$
\left|\mathbf{M}_{\mathrm{B}}^{(k)}\right|=\left|\begin{array}{ccc}
\mathbf{M}_{\mathrm{B}, 11} & \cdots & \mathbf{M}_{\mathrm{B}, k 1} \\
\vdots & \ddots & \vdots \\
\mathbf{M}_{\mathrm{B}, 1 k} & \cdots & \mathbf{M}_{\mathrm{B}, k k}
\end{array}\right| .
$$

of order $k=3, \cdots, M+1$ has a sign other than -1 . 


\section{Examples}

In this section, we show two application examples with explicit formulation of invasion fitness functions built from resource competition. In the first example, we show how our method works by analyzing a simple two-dimensional case. Then, we analyze its higher-dimensional extension in the second example.

\subsection{Example 1: Evolutionary branching along a constraint curve in a two-dimensional resource competition model}

\section{Model}

We consider a two-dimensional trait space $\mathbf{s}=(x, y)^{\mathrm{T}}$, which is treated as a twodimensional niche space with two niche axes $x$ and $y$. We assume a constraint curve

$$
h\left(\mathbf{s}^{\prime}\right)=y^{\prime}-\frac{a}{2} x^{\prime 2}+b=0,
$$

which is a parabolic curve $y^{\prime}=\frac{a}{2} x^{\prime 2}-b$ with two constant parameters $a$ and $b$ (solid curves in Fig. 3).

The invasion fitness function is constructed in the two-dimensional MacArthur-Levins resource competition model (Vukics et al., 2003), explained below. When there exist $N$-phenotypes, the $i$ th phenotype's growth rate is defined by the Lotka-Volterra competition model,

$$
\frac{d n_{i}}{d t}=n_{i}\left[1-\sum_{j=1}^{N} \frac{\alpha\left(\mathbf{s}_{i} ; \mathbf{s}_{j}\right) n_{j}}{K\left(\mathbf{s}_{i}\right)}\right],
$$

where carrying capacity $K\left(\mathbf{s}_{i}\right)$ of $\mathbf{s}_{i}$ and the competition effect $\alpha\left(\mathbf{s}_{i} ; \mathbf{s}_{j}\right)$ on $\mathbf{s}_{i}$ from $\mathbf{s}_{j}$ are both given by two-dimensional isotropic Gaussian distributions

$$
\begin{gathered}
K\left(\mathbf{s}_{i}\right)=K_{0} \exp \left(-\frac{\left|\mathbf{s}_{i}\right|^{2}}{2 \sigma_{\mathrm{K}}^{2}}\right), \\
\alpha\left(\mathbf{s}_{i} ; \mathbf{s}_{j}\right)=\exp \left(-\frac{\left(\left|\mathbf{s}_{i}-\mathbf{s}_{j}\right|^{2}\right)}{2 \sigma_{\alpha}^{2}}\right),
\end{gathered}
$$

where $K\left(\mathbf{s}_{i}\right)$ has its peak $K_{0}$ at the origin with standard deviation $\sigma_{\mathrm{K}}$, and $\alpha\left(\mathbf{s}_{i} ; \mathbf{s}_{j}\right)$ has its peak 1 at $\mathbf{s}_{i}=\mathbf{s}_{j}$ with standard deviation $\sigma_{\alpha}$, i.e., the competition effect decreases with their phenotypic distance. As this model and the constraint curve Eq. (25) are both symmetric about the $y$-axis, we focus only on positive $x$ without loss of generality. 


\section{Analysis of evolutionary branching}

We suppose a resident $\mathbf{s}^{\circ}$ and a mutant $\mathbf{s}^{\prime}$ with population densities $n^{\circ}$ and $n^{\prime}$, respectively. The invasion fitness $F\left(\mathbf{s}^{\prime} ; \mathbf{s}^{\circ}\right)$ of $\mathbf{s}^{\prime}$ against $\mathbf{s}^{\circ}$ is defined by its initial growth rate (i.e., when $n^{\prime}$ is very small) in the resident population at equilibrium density $n^{\circ}=K\left(\mathbf{s}^{\circ}\right)$,

$$
F\left(\mathbf{s}^{\prime} ; \mathbf{s}^{\circ}\right)=\lim _{n^{\prime} \rightarrow+0}\left[\frac{1}{n^{\prime}} \frac{\mathrm{d} n^{\prime}}{\mathrm{d} t}\right]_{n^{\circ}=K\left(\mathbf{s}^{\circ}\right)}=1-\frac{\alpha\left(\mathbf{s}^{\prime} ; \mathbf{s}^{\circ}\right) K\left(\mathbf{s}^{\circ}\right)}{K\left(\mathbf{s}^{\prime}\right)} .
$$

The first and second derivatives of this fitness function at an arbitrary point $\mathbf{s}$ give

$$
\begin{aligned}
& \mathbf{g}=\nabla_{\mathbf{s}^{\prime}} F(\mathbf{s} ; \mathbf{s})=-\frac{1}{\sigma_{\mathrm{K}}^{2}}\left(\begin{array}{l}
x \\
y
\end{array}\right), \\
& \mathbf{D}=\nabla_{\mathbf{s}^{\prime}} \nabla_{\mathbf{s}^{\prime}}^{\mathrm{T}} F(\mathbf{s} ; \mathbf{s})=\left[\frac{1}{\sigma_{\alpha}^{2}}-\frac{1}{\sigma_{\mathrm{K}}^{2}}\right]\left(\begin{array}{cc}
1 & 0 \\
0 & 1
\end{array}\right)-\frac{1}{\sigma_{\mathrm{K}}^{4}}\left(\begin{array}{ll}
x^{2} & x y \\
x y & y^{2}
\end{array}\right), \\
& \mathbf{C}=\left(\nabla_{\mathbf{s}^{\prime}} \nabla_{\mathbf{s}^{\prime}}^{\mathrm{T}}+\nabla_{\mathbf{s}^{\circ}} \nabla_{\mathbf{s}^{\prime}}^{\mathrm{T}}\right) F(\mathbf{s} ; \mathbf{s})=-\frac{1}{\sigma_{\mathrm{K}}^{2}}\left(\begin{array}{ll}
1 & 0 \\
0 & 1
\end{array}\right),
\end{aligned}
$$

and the derivatives of the constraint curve

$$
\begin{aligned}
\nabla h(\mathbf{s}) & =\left(\begin{array}{c}
-a x \\
1
\end{array}\right), \\
\nabla \nabla^{\mathrm{T}} h(\mathbf{s}) & =\left(\begin{array}{cc}
-a & 0 \\
0 & 0
\end{array}\right)
\end{aligned}
$$

give its normal, tangent, and curvature vectors at $\mathbf{s}$

$$
\begin{aligned}
& \mathbf{n}=\frac{\nabla h(\mathbf{s})}{|\nabla h(\mathbf{s})|}=\frac{1}{\sqrt{a^{2} x^{2}+1}}\left(\begin{array}{c}
-a x \\
1
\end{array}\right), \\
& \mathbf{e}=\frac{1}{\sqrt{a^{2} x^{2}+1}}\left(\begin{array}{c}
1 \\
a x
\end{array}\right), \\
& \mathbf{q}=-\frac{\mathbf{e}^{\mathrm{T}} \nabla \nabla^{\mathrm{T}} h(\mathbf{s}) \mathbf{e}}{|\nabla h(\mathbf{s})|} \mathbf{n}=\frac{a}{\sqrt{\left(a^{2} x^{2}+1\right) \mathbf{n}}} .
\end{aligned}
$$

The Lagrange fitness function is constructed as

$$
\begin{aligned}
F_{\mathrm{L}}\left(\mathbf{s}^{\prime} ; \mathbf{s}^{\circ} ; \lambda_{\mathbf{s}}\right) & =F\left(\mathbf{s}^{\prime} ; \mathbf{s}^{\circ}\right)-\lambda_{\mathbf{s}}\left[h\left(\mathbf{s}^{\prime}\right)-h\left(\mathbf{s}^{\circ}\right)\right] \\
& =1-\frac{\alpha\left(\mathbf{s}^{\prime} ; \mathbf{s}^{\circ}\right) K\left(\mathbf{s}^{\circ}\right)}{K\left(\mathbf{s}^{\prime}\right)}-\lambda_{\mathbf{s}}\left[h\left(\mathbf{s}^{\prime}\right)-h\left(\mathbf{s}^{\circ}\right)\right]
\end{aligned}
$$

with 


$$
\lambda_{\mathbf{s}}=\frac{\nabla h(\mathbf{s}) \cdot \mathbf{g}}{|\nabla h(\mathbf{s})|^{2}}=\frac{\left(a x^{2}-y\right)}{\sigma_{\mathrm{K}}^{2}\left(a^{2} x^{2}+1\right)} .
$$

To apply Theorem 1 , we calculate $\nabla_{\mathbf{s}^{\prime}} F_{\mathrm{L}}(\mathbf{s} ; \mathbf{s} ; \lambda), C_{\mathrm{h}}$, and $D_{\mathrm{h}}$ as

$$
\begin{aligned}
\nabla_{\mathbf{s}^{\prime}} F_{\mathrm{L}}\left(\mathbf{s} ; \mathbf{s} ; \lambda_{\mathbf{s}}\right) & =\mathbf{g}-\lambda_{\mathbf{s}} \nabla h(\mathbf{s})=-\frac{x(1+a y)}{\sigma_{\mathrm{K}}^{2}\left(a^{2} x^{2}+1\right)}\left(\begin{array}{c}
1 \\
a x
\end{array}\right), \\
C_{\mathrm{h}} & =\mathbf{e}^{\mathrm{T}}\left[\mathbf{C}-\lambda_{\mathbf{s}} \nabla \nabla^{\mathrm{T}} h(\mathbf{s})\right] \mathbf{e}=\frac{1}{\sigma_{\mathrm{K}}^{2}}\left[\frac{a^{2} x^{2}-a y}{\left(a^{2} x^{2}+1\right)^{2}}-1\right], \\
D_{\mathrm{h}} & =\mathbf{e}^{\mathrm{T}}\left[\mathbf{D}-\lambda_{\mathbf{s}} \nabla \nabla^{\mathrm{T}} h(\mathbf{s})\right] \mathbf{e} \\
& =\frac{1}{\sigma_{\mathrm{K}}^{2}}\left[\frac{\sigma_{\mathrm{K}}^{2}}{\sigma_{\alpha}^{2}}-1-\frac{1}{\sigma_{\mathrm{K}}^{2}} \frac{x^{2}(a y+1)^{2}}{\left(a^{2} x^{2}+1\right)}+\frac{a^{2} x^{2}-a y}{\left(a^{2} x^{2}+1\right)^{2}}\right] .
\end{aligned}
$$

$C_{\mathrm{h}}$ and $D_{\mathrm{h}}$ can also be obtained from bordered second-derivative matrices [Eqs. (22b) and (22d)].

By condition (i) in Theorem 1, the condition for evolutionary singularity along the curve is given by

$$
\nabla_{\mathbf{s}^{\prime}} F_{\mathrm{L}}\left(\mathbf{s} ; \mathbf{s} ; \lambda_{\mathbf{s}}\right)=-\frac{x(1+a y)}{\sigma_{\mathrm{K}}^{2}\left(a^{2} x^{2}+1\right)}\left(\begin{array}{c}
1 \\
a x
\end{array}\right)=\mathbf{0},
$$

which yields two singular points

$$
\mathbf{s}_{1}=\left(\begin{array}{c}
0 \\
-b
\end{array}\right), \quad \mathbf{s}_{2}=\left(\begin{array}{c}
\frac{\sqrt{2(a b-1)}}{a} \\
-\frac{1}{a}
\end{array}\right)
$$

( $\mathbf{s}_{1}$ and $\mathbf{s}_{2}$ can also be obtained by Eq. (11), which may be easier). $\mathbf{s}_{2}$ can exist only when $a b>1$. The condition $a b>1$ is understood as follows. The radius of the curvature of the constraint curve, given by $1 /|\mathbf{q}|$, has its minimum $1 / a$ at $\mathbf{s}_{1}$, whereas that of its tangential contour curve of $K(\mathbf{s}), x^{2}+y^{2}=b$, is constant $b$. Thus, they have only a single tangent point $\mathbf{s}_{1}$ for $1 / a>b$ (Fig. 3a), but two tangent points $\mathbf{s}_{1}$ and $\mathbf{s}_{2}$ for $1 / a<b$ (Fig. $3 b$ ).

Condition (ii) in Theorem 1 applied to each of two singular points defined above gives the conditions for their convergence stability along the constraint curve,

$$
\begin{gathered}
C_{\mathrm{h} 1}=\frac{1}{\sigma_{\mathrm{K}}^{2}}(a b-1)<0, \\
C_{\mathrm{h} 2}=-\frac{2(a b-1)}{\sigma_{\mathrm{K}}^{2}(2 a b-1)}<0,
\end{gathered}
$$


respectively, and condition (iii) gives the conditions for their evolutionary instability along the curve,

$$
\begin{gathered}
D_{\mathrm{h} 1}=\frac{1}{\sigma_{\alpha}^{2}}-\frac{1-a b}{\sigma_{\mathrm{K}}^{2}}>0, \\
D_{\mathrm{h} 2}=\frac{1}{\sigma_{\alpha}^{2}}+\frac{1}{\sigma_{\mathrm{K}}^{2}}\left[\frac{1}{2 a b-1}-1\right]>0,
\end{gathered}
$$

respectively. Clearly, when $a b<1$, the unique singular point $\mathbf{s}_{1}$ is always convergence stable. Moreover, this point is an evolutionary branching point as long as $a b$ is sufficiently close to 1 , because Eq. (36a) is transformed into

$$
\left(\frac{\sigma_{\mathrm{K}}}{\sigma_{\alpha}}\right)^{2}>1-a b
$$

(region A in Fig. 4). When $a b>1$, there exist two singular points $\mathbf{s}_{1}$ and $\mathbf{s}_{2}$, in which case $\mathbf{s}_{2}$ is always convergence stable while $\mathbf{s}_{1}$ never is. By Eq. (36b), $\mathbf{s}_{2}$ is an evolutionary branching point when

$$
\left(\frac{\sigma_{\mathrm{K}}}{\sigma_{\alpha}}\right)^{2}>1-\frac{1}{2 a b-1}
$$

(region C in Fig. 4).

Notice that evolutionary branching points exist even for $\sigma_{\mathrm{K}} / \sigma_{\alpha}<1$ as long as $a b$ is sufficiently close to 1 (i.e., when the constraint curve and its tangential contour of $K(\mathbf{s})$ have sufficiently similar curvature radii of at $\left.\mathbf{s}_{1}\right)$. Conversely, when the constraint curve is a straight line $(a=0)$, evolutionary branching points can exist only when $\sigma_{\mathrm{K}} / \sigma_{\alpha}>1$, equivalent to the case of one-dimensional trait spaces with no constraint (Dieckmann and Doebeli, 1999).

\subsection{Example 2: Potential for evolutionary branching through resource competition in multi-dimensional trait spaces}

We generalize the above two-dimensional model and apply the branching potential condition to determine whether each point in the trait space can become a CBP when we freely choose the shape of the constraint surface.

\section{Model}

We consider an arbitrary $M$-dimensional trait space $\mathbf{s}=\left(x_{1}, \cdots, x_{M}\right)^{\mathrm{T}}$, where the growth rate of phenotype $\mathbf{s}_{i}$ is given by the same equation used for twodimensional resource competition [Eq. (26a)], which gives the same form of the invasion fitness function 


$$
F\left(\mathbf{s}^{\prime} ; \mathbf{s}^{\circ}\right)=\lim _{n^{\prime} \rightarrow+0}\left[\frac{1}{n^{\prime}} \frac{d n^{\prime}}{d t}\right]_{n^{\circ}=K\left(\mathbf{s}^{\circ}\right)}=1-\frac{\alpha\left(\mathbf{s}^{\prime} ; \mathbf{s}^{\circ}\right) K\left(\mathbf{s}^{\circ}\right)}{K\left(\mathbf{s}^{\prime}\right)} .
$$

Unlike the two-dimensional case, we do not define explicit forms for the carrying capacity distribution $K(\mathbf{s})$ and competition kernel $\alpha\left(\mathbf{s}^{\prime} ; \mathbf{s}^{\circ}\right)$. We assume that those functions are both smooth. For the competition kernel, we assume that $\alpha\left(\mathbf{s}^{\circ} ; \mathbf{s}^{\circ}\right)=1$, and that competition strength is determined by the relative phenotypic difference of $\mathbf{s}^{\prime}$ from $\mathbf{s}^{\circ}$, i.e., $\alpha\left(\mathbf{s}^{\prime} ; \mathbf{s}^{\circ}\right)$ can be treated as a function with a single argument $\mathbf{s}^{\prime}-\mathbf{s}^{\circ}$,

$$
\alpha\left(\mathbf{s}^{\prime} ; \mathbf{s}^{\circ}\right)=\tilde{\alpha}\left(\mathbf{s}^{\prime}-\mathbf{s}^{\circ}\right) .
$$

We also assume that the strength of competition is maximal between identical phenotypes, i.e.,

$$
\nabla_{\mathbf{s}^{\prime}} \alpha\left(\mathbf{s}^{\circ} ; \mathbf{s}^{\circ}\right)=\mathbf{0}
$$

and the symmetric matrix

$$
\nabla_{\mathbf{s}^{\prime}} \nabla_{\mathbf{s}^{\prime}}^{\mathrm{T}} \alpha\left(\mathbf{s}^{\circ} ; \mathbf{s}^{\circ}\right)
$$

is negative definite for any $\mathbf{s}^{\circ}$. For example, the Gaussian competition kernel in the two-dimensional model given by Eq. (26b) fulfills these conditions.

\section{Potential for evolutionary branching}

At an arbitrary point $\mathbf{s}$, the first and second derivatives of the invasion fitness are obtained as

$$
\begin{aligned}
\mathbf{g} & =\nabla_{\mathbf{s}^{\prime}} F(\mathbf{s} ; \mathbf{s})=\nabla \ln K(\mathbf{s}), \\
\mathbf{C} & =\nabla_{\mathbf{s}^{\prime}} \nabla_{\mathbf{s}^{\prime}}^{\mathrm{T}} F(\mathbf{s} ; \mathbf{s})+\nabla_{\mathbf{s}^{\circ}} \nabla_{\mathbf{s}^{\prime}}^{\mathrm{T}} F(\mathbf{s} ; \mathbf{s})=\nabla \nabla^{\mathrm{T}} \ln K(\mathbf{s}), \\
\mathbf{D} & =\nabla_{\mathbf{s}^{\prime}} \nabla_{\mathbf{s}^{\prime}}^{\mathrm{T}} F(\mathbf{s} ; \mathbf{s}) \\
& =-\nabla_{\mathbf{s}^{\prime}} \nabla_{\mathbf{s}^{\prime}}^{\mathrm{T}} \alpha(\mathbf{s} ; \mathbf{s})+\nabla \nabla^{\mathrm{T}} \ln K(\mathbf{s})-\nabla \ln K(\mathbf{s}) \nabla^{\mathrm{T}} \ln K(\mathbf{s}), \\
\mathbf{M} & =-\nabla_{\mathbf{s}^{\circ}} \nabla_{\mathbf{s}^{\prime}}^{\mathrm{T}} F(\mathbf{s} ; \mathbf{s})=\mathbf{D}-\mathbf{C} \\
& =-\nabla_{\mathbf{s}^{\prime}} \nabla_{\mathbf{s}^{\prime}}^{\mathrm{T}} \alpha(\mathbf{s} ; \mathbf{s})-\nabla \ln K(\mathbf{s}) \nabla^{\mathrm{T}} \ln K(\mathbf{s})
\end{aligned}
$$

(Appendix H). Then, by the branching potential condition in Theorem 3, we can quickly examine whether an arbitrary point $\mathbf{s}$ has potential for being a CBP. In this model, the branching potential matrix (Eq. (24a)) is calculated as 


$$
\begin{aligned}
\mathbf{P}=\frac{1}{2} \mathbf{U}^{\mathrm{T}}\left[\mathbf{M}+\mathbf{M}^{\mathrm{T}}\right] \mathbf{U} & =-\mathbf{U}^{\mathrm{T}}\left[\nabla_{\mathbf{s}^{\prime}} \nabla_{\mathbf{s}^{\prime}}^{\mathrm{T}} \alpha(\mathbf{s} ; \mathbf{s})+\mathbf{g g}^{\mathrm{T}}\right] \mathbf{U} \\
& =-\mathbf{U}^{\mathrm{T}} \nabla_{\mathbf{s}^{\prime}} \nabla_{\mathbf{s}^{\prime}}^{\mathrm{T}} \alpha(\mathbf{s} ; \mathbf{s}) \mathbf{U}+\mathbf{U}^{\mathrm{T}} \mathbf{g g} \mathbf{T}^{\mathrm{T}} \mathbf{U} \\
& =-\mathbf{U}^{\mathrm{T}} \nabla_{\mathbf{s}^{\prime}} \nabla_{\mathbf{s}^{\prime}}^{\mathrm{T}} \alpha(\mathbf{s} ; \mathbf{s}) \mathbf{U},
\end{aligned}
$$

where $\mathbf{U}^{\mathrm{T}} \mathbf{g}=\mathbf{g}^{\mathrm{T}} \mathbf{U}=\mathbf{g}^{\mathrm{T}}\left[\mathbf{I}-\mathbf{g g}^{\mathrm{T}} /|\mathbf{g}|^{2}\right]=\mathbf{g}^{\mathrm{T}}-\mathbf{g}^{\mathrm{T}}=\mathbf{0}$ is used. As $\nabla_{\mathbf{s}^{\prime}} \nabla_{\mathbf{s}^{\prime}}^{\mathrm{T}} \alpha(\mathbf{s} ; \mathbf{s})$ is assumed to be negative definite, Eq. (41) is positive semidefinite, i.e., $\mathbf{v}^{\mathrm{T}} \mathbf{P v}$ is zero for $\mathbf{v} \propto \mathbf{g}$, or positive otherwise. Thus, Eq. (41) has $(M-1)$ positive eigenvalues and a single zero eigenvalue in the direction of $\mathbf{g}$. Therefore, any $\mathbf{s}$ can become a CBP with the appropriate choice of local dimensionality and shape of the constraint surface around the point. Such a constraint surface is given by substituting Eq. (40) into Eq. (24b), yielding

$$
\begin{aligned}
h\left(\mathbf{s}^{\prime}\right)= & {[\nabla \ln K(\mathbf{s})]^{\mathrm{T}}\left[\mathbf{s}^{\prime}-\mathbf{s}\right] } \\
& +\frac{1}{2}\left[\mathbf{s}^{\prime}-\mathbf{s}\right]^{\mathrm{T}}\left[\nabla \nabla^{\mathrm{T}} \ln K(\mathbf{s})+\tilde{\varepsilon} \mathbf{I}\right]\left[\mathbf{s}^{\prime}-\mathbf{s}\right]+\mathrm{O}\left(\left|\mathbf{s}^{\prime}-\mathbf{s}\right|^{3}\right) \\
& =\ln K\left(\mathbf{s}^{\prime}\right)+\frac{\tilde{\varepsilon}}{2}\left|\mathbf{s}^{\prime}-\mathbf{s}\right|^{2}+\mathrm{O}\left(\left|\mathbf{s}^{\prime}-\mathbf{s}\right|^{3}\right)=0,
\end{aligned}
$$

which gives

$$
\begin{aligned}
\nabla h(\mathbf{s}) & =\nabla \ln K(\mathbf{s}), \\
\nabla \nabla^{\mathrm{T}} h(\mathbf{s}) & =\nabla \nabla^{\mathrm{T}} \ln K(\mathbf{s})+\tilde{\varepsilon} \mathbf{I},
\end{aligned}
$$

with a positive and sufficiently small $\tilde{\varepsilon}$. In other words, for an $(M-1)$ -

dimensional constraint surface with a tangent point $\mathbf{s}$ of an isosurface of $K(\mathbf{s})$, if the constraint surface has slightly weaker curvature (by $\tilde{\varepsilon}$ ) than the isosurface at $\mathbf{s}$, then $\mathbf{s}$ is a CBP along the surface, as illustrated in Figure 5.

\section{Multi-dimensional Lagrange multiplier method}

Although Appendix G proves that Eq. (42) makes s become a CBP along the constraint surface in a general way, here we directly apply Theorem 2 to Eq. (42) and show how this theorem works. As the constraint surface has only a single equality condition $h\left(\mathbf{s}^{\prime}\right)=0$, the Lagrange fitness function [Eq. (15)] for a focal point $\mathbf{s}$ becomes

$$
F_{\mathrm{L}}\left(\mathbf{s}^{\prime} ; \mathbf{s}^{\circ} ; \lambda_{\mathbf{s}}\right)=F\left(\mathbf{s}^{\prime} ; \mathbf{s}^{\circ}\right)-\lambda_{\mathbf{s}}\left[h\left(\mathbf{s}^{\prime}\right)-h\left(\mathbf{s}^{\circ}\right)\right],
$$

with a scalar $\lambda_{\mathbf{s}}$ given by Eq. (19)

$$
\lambda_{\mathbf{s}}=\mathbf{B}^{+} \nabla_{\mathbf{s}^{\prime}} F(\mathbf{s} ; \mathbf{s})=\frac{\nabla^{\mathrm{T}} \ln K(\mathbf{s})}{|\nabla \ln K(\mathbf{s})|^{2}} \nabla \ln K(\mathbf{s})=1,
$$


where $\mathbf{B}=\nabla h(\mathbf{s})$ and $\mathbf{B}^{+}=\left[\mathbf{B}^{\mathrm{T}} \mathbf{B}\right]^{-1} \mathbf{B}^{\mathrm{T}}=\nabla^{\mathrm{T}} h(\mathbf{s}) /|\nabla h(\mathbf{s})|^{2}=\nabla^{\mathrm{T}} \ln K(\mathbf{s}) /$ $|\nabla \ln K(\mathbf{s})|^{2}$ are column and row vectors, respectively. As for the choice of base vectors for the tangent plane of the constraint surface, we can use the eigenvectors corresponding to positive eigenvalues of the branching potential matrix [Eq. (41)] as the orthogonal base vectors, $\mathbf{E}=\left(\mathbf{e}_{1}, \ldots, \mathbf{e}_{M-1}\right)$, satisfying $\mathbf{e}_{i} \cdot \mathbf{n}=0$ for all $i=$ $1, \ldots, M-1$, where $\mathbf{n}=\nabla h(\mathbf{s}) /|\nabla h(\mathbf{s})|$ is the normal vector of the surface.

Then, by condition (i) in Theorem 2, any $\mathbf{s}$ is evolutionarily singular along the surface, as it satisfies

$$
\begin{aligned}
\nabla_{\mathbf{s}^{\prime}} F_{\mathrm{L}}\left(\mathbf{s} ; \mathbf{s} ; \lambda_{\mathbf{s}}\right) & =\nabla \ln K(\mathbf{s})-\lambda_{\mathbf{s}} \nabla h(\mathbf{s}) \\
& =\nabla \ln K(\mathbf{s})-\nabla \ln K(\mathbf{s})=\mathbf{0} .
\end{aligned}
$$

As for condition (ii), we calculate

$$
\begin{aligned}
\mathbf{C}_{\mathrm{h}} & =\mathbf{E}^{\mathrm{T}}\left[\mathbf{C}-\lambda_{\mathbf{s}} \nabla \nabla^{\mathrm{T}} h(\mathbf{s})\right] \mathbf{E} \\
& =\mathbf{E}^{\mathrm{T}}\left[\nabla \nabla^{\mathrm{T}} \ln K(\mathbf{s})-\nabla \nabla^{\mathrm{T}} \ln K(\mathbf{s})-\tilde{\varepsilon} \mathbf{I}\right] \mathbf{E} \\
& =-\tilde{\varepsilon} \mathbf{E}^{\mathrm{T}} \mathbf{E}=-\tilde{\varepsilon} \mathbf{I}_{M-1} .
\end{aligned}
$$

Thus, $\frac{1}{2}\left[\mathbf{C}_{\mathrm{h}}+\mathbf{C}_{\mathrm{h}}^{\mathrm{T}}\right]=\mathbf{C}_{\mathrm{h}}=-\tilde{\varepsilon}_{M-1}$ is always negative definite with positive $\tilde{\varepsilon}$, in which case $\mathbf{S}$ is always strongly convergence stable along the constraint surface. Condition (iii) gives its evolutionary stability condition

$$
\begin{aligned}
\mathbf{D}_{\mathrm{h}} & =\mathbf{E}^{\mathrm{T}}\left[\mathbf{D}-\lambda_{\mathbf{s}} \nabla \nabla^{\mathrm{T}} h(\mathbf{s})\right] \mathbf{E} \\
& =-\mathbf{E}^{\mathrm{T}}\left[\nabla_{\mathbf{s}^{\prime}} \nabla_{\mathbf{s}^{\prime}}^{\mathrm{T}} \alpha(\mathbf{s} ; \mathbf{s})+\nabla \ln K(\mathbf{s}) \nabla^{\mathrm{T}} \ln K(\mathbf{s})+\tilde{\varepsilon} \mathbf{I}\right] \mathbf{E} \\
& =-\mathbf{E}^{\mathrm{T}}\left[\nabla_{\mathbf{s}^{\prime}} \nabla_{\mathbf{s}^{\prime}}^{\mathrm{T}} \alpha(\mathbf{s} ; \mathbf{s})+\tilde{\varepsilon} \mathbf{I}\right] \mathbf{E},
\end{aligned}
$$

where $\mathbf{E}^{\mathrm{T}} \nabla \ln K(\mathbf{s})=\mathbf{E}^{\mathrm{T}} \mathbf{g}=\mathbf{0}$ is used. As $\nabla_{\mathbf{s}^{\prime}} \nabla_{\mathbf{s}^{\prime}}^{\mathrm{T}} \alpha(\mathbf{s} ; \mathbf{s})$ is negative definite by definition, $\mathbf{D}_{\mathrm{h}}$ is positive definite for sufficiently small $\tilde{\varepsilon}$. Therefore, $\mathbf{s}$ is a CBP along the constraint surface for positive and sufficiently small $\tilde{\varepsilon}$. As $\mathbf{C}_{\mathrm{h}}$ and $\mathbf{D}_{\mathrm{h}}$ are negative definite and positive definite, respectively, in this case, any smooth subspace of this constraint surface that contains $\mathbf{s}$ also has a CBP at $\mathbf{s}$.

\section{Discussion}

\subsection{Extension of Levins' fitness set theory}

Adaptive evolution is multi-dimensional in nature, and it is a widespread phenomenon that evolutionary constraints (e.g., due to genetic, developmental, physiological, or physical constraints) restrict directions that allow mutants to emerge or to have sufficient fertility (Flatt and Heyland, 2011). For example, 
genotypes of a zooplankton species (Daphnia dentifera) illustrate the trade-off between feeding speed and efficiency (Hall et al., 2012). This situation may be proximately due to genetic or developmental systems, but it might ultimately be imposed by physical laws because no system can maximize power and efficiency at the same time under the second law of thermodynamics. Due to those constraints, an evolutionary trajectory induced by selection may be bounded on subspaces with fewer dimensionalities (e.g., selection responses of butterfly wing spots (Allen et al., 2008)). If such a subspace, i.e., a constraint surface, is parameterized so that coordinates on the surface are described with those parameters, adaptive evolution along the surfaces can be translated into adaptive evolution in the parameter space without constraint. In such a case, conventional analysis of parameters such as directional selection, evolutionary stability, and convergence stability can apply directly. However, parameterization may be difficult or complicated when the constraint surfaces are multi-dimensional.

Levins $(1962,1968)$ developed a geometric method for the analysis of adaptive evolution along constraint curves (or surfaces), which does not require their parameterization. This method, known as 'Levins' fitness set theory,' can be used to analyze directional evolution and evolutionarily stable points along constraint curves by examining how the contours of fitness landscapes in the trait spaces cross or are tangent to the constraint curves. A limitation of this method is that fitness functions are assumed to be independent of existing resident phenotypes, i.e., frequency-independent, despite the expectation of such dependency in fundamental ecological interactions (e.g., resource competition, predator-prey interactions, mutualism) (Dieckmann et al., 2004). In this case, the resulting static fitness landscape cannot induce evolutionary branching (Metz et al., 1996; Geritz et al., 1997, 1998), although evolutionary branching is thought to be an important ecological mechanism for the evolutionary diversification of biological communities (Dieckmann et al., 2004).

Recently, Levins' method has been extended to the analysis of frequencydependent fitness functions for one-dimensional constraint curves in twodimensional trait spaces (Rueffler et al. 2004; deMazancourt and Dieckmann, 2004; Bowers et al. 2005). The extended method can be used to analyze evolutionary branching along constraint curves by examining convergence stability as well as the evolutionary singularity and stability of focal points.

In this paper, we further developed the extension of Levins' method described above to analyze constraint surfaces of arbitrary dimensionalities in the form of Lagrange multiplier method. As our Lagrange multiplier method is completely analytic, one can easily use it to analyze adaptive evolution along constraint 
surfaces of arbitrary dimensionalities without imaging them graphically. This feature may also be useful in numerical analysis. The core operation of our method is local parameterization of the constraint surface by using its tangent plane as the parameter space [Eq. (B.4) in Appendix B and Eq. (D.7) in Appendix D]. As this operation is performed in the simple procedure of making Lagrange fitness functions [Eqs. (15) and (19)], no explicit coordinate transformation is required, which enables efficient analysis. Our method is readily extended to infinitedimensional trait spaces, called function-valued traits, such as resource utilization distributions on continuous resource-quality axes and energy allocations to different organs or functions on a continuous time axis (Dieckmann et al. 2006; Parvinen et al. 2013). By this infinite-dimensional extension, the analysis of convergence stability in function-valued traits becomes more efficient (Ito and Sasaki , in preparation).

\subsection{Conditions for evolutionary branching in multi- dimensional trait spaces}

In this paper, we refer to points that are strongly convergence stable and evolutionarily unstable in multi-dimensional trait spaces as CBPs. Those two conditions, respectively, ensure that monomorphic populations converge to points and that mutants still can invade against residents located at the points. However, whether they can coexist and evolutionarily diversify into distinct morphs, called 'dimorphic emergence' and 'dimorphic divergence,' respectively, in Ito and Dieckmann (2014), is not clear. Geritz et al. (2016) proved that dimorphic emergence is ensured at CBPs in trait spaces of arbitrary dimensionality. As for dimorphic divergence, Geritz et al. (2016) provided a set of conditions ensuring that any initial small-scale polymorphism around CBPs results in diversifying evolution toward distinct dimorphism, where their directional coevolution are described with coupled Lande equations. As those conditions imply that morphs diversify sufficiently faster than their mean moves (Geritz et al. 2016), we refer to the condition as the 'divergence-speed condition' in this paper. In two-dimensional trait spaces, CBPs satisfy this condition, i.e., CBPs can be treated as evolutionary branching points (Geritz et al. 2016). In higher-dimensional trait spaces, however, whether any CBP satisfies the divergence-speed condition remains unclear (Geritz et al. 2016). Therefore, whether any CBP ensures evolutionary branching remains an open question. 


\subsection{Mutations}

In our analysis, we assume that mutation never occurs in directions orthogonal to the constraint surfaces. In reality, however, such mutations can occur, although their mutation rates may be very low and/or their mutational step sizes may be very small. If there exists a fitness gradient toward those orthogonal directions, the constraint surface itself may evolve directionally at a very slow speed. As long as directional selection along the constraint surfaces is not weak, such slow evolution of the surface can be neglected. On the other hand, when populations have come close to an evolutionarily singular point where directional selection along the surface becomes very weak, subsequent dynamics, including evolutionary branching, may be affected seriously by the slow evolution of the constraint surface. Conditions for evolutionary branching in this situation have been developed for flat constraint surfaces (Ito and Dieckmann 2007; 2012; 2014). Application of those conditions by extending our Lagrange multiplier method allows us to examine how the shapes of constraint surfaces and their slow evolution affect the likelihood of evolutionary branching along surfaces (Ito and Sasaki, in preparation).

\subsection{Branching potential conditions}

The evolutionary trajectories of species in a genus or a family may be expressed in a single multi-dimensional trait space, by assuming a sufficiently large number of trait axes. In the trait space, closely related species may share the same constraint surface (Schluter, 1996), whereas distant species may have different constraint surfaces due to those surfaces' slow evolution, as mentioned above. We may then ask whether the trait space has regions that always favor (or always suppress) evolutionary diversification, irrespective of the shapes of constraint surfaces, or favor diversification only for particular shapes. When a fitness function for the trait space is given and the constraint is one-dimensional (i.e., constraint curves), this question can be addressed by analyzing each position of the trait space. The analysis examines the condition by which the point becomes an evolutionary branching point by adjusting the shapes of the constraint curves (Bowers et al. 2003, 2005; Rueffler et al. 2004; deMazancourt and Dieckmann, 2004; Kisdi, 2006, 2015). In this paper, we extended this condition to multi-dimensional constraint surfaces, and referred to it as a branching potential condition.

The branching potential condition is particularly useful when we want to know whether a focal ecological interaction embedded in a mathematical model has the potential to induce evolutionary branching by adjusting all of the remaining ingredients of the model. By treating all constants and variables as 
additional traits, and adding them to the original trait space, we can use the branching potential condition to examine whether each position in the hyper-trait space has the potential to be an evolutionary branching point. If we find that points have branching potential, then their positions and the obtained local shapes of constraint surfaces indicate how we can adjust the model to induce evolutionary branching. If the model is general, so that it covers a sufficiently wide range of life histories, this analysis may reveal the potential of the focal ecological interaction itself for inducing evolutionary diversification.

Our branching potential condition corresponds to an extension of the 'direct analysis' for one-dimensional, parameterized constraint curves in Kisdi (2015). While our condition ensures CBPs on multi-dimensional constraint surfaces, the condition itself is mathematically equivalent to Kisdi's condition. Kisdi (2015) also derived a condition for branching potential in terms of environmental feedback variables, which are variables through which resident phenotypes affect the invasion fitness of mutants (e.g., densities of different types of resource and predator). The environmental feedbacks are the source of frequency-dependent selection, and their effective number yields the maximum number of residents that can coexist in a system (Meszéna and Metz 1999; Meszéna et al., 2006; Metz et al., 2008). Thus, by analyzing environmental variables, one may gain essential insight about evolutionary dynamics that potentially arise in the system (e.g., if the environmental feedback dimension is one, then evolutionary branching is impossible). Kisdi (2015) has shown that any combination of convergence stability and evolutionary stability can be realized for an arbitrary point in a trait space by choosing an appropriate one-dimensional constraint curve containing it, as long as the local region around the point has at least two effective environmental feedbacks and the dimension of the trait space is more than the number of feedbacks under certain non-degeneracy conditions (e.g., neither trait can be neutral). Those conditions are sufficient (but not necessary) for the branching potential matrix in this paper to have both positive and negative eigenvalues (because the transpose of $\mathbf{M}$ in Eq. (24c) is identical to $\mathbf{S E}$ in Eq. (6) in Kisdi (2015)). While this condition seems important, some models may not satisfy both of the non-degeneracy conditions prohibiting neutral traits and the dimensionality condition requiring that the number of feedbacks is smaller than the trait space dimension. Thus, a future step would be to extend Kisdi's condition to make it closer to a necessary and sufficient one for our branching potential condition (or Kisdi's direct condition, equivalently), so that the potential of evolutionary branching along constraint surfaces is understood fully in terms of environmental feedbacks. 


\section{Acknowledgements}

The authors thank to the two anonymous reviewers and editors for valuable comments on earlier versions of this manuscript. This study was supported in part by the MEXT Grant-in-Aid for Scientific Research on Innovative Areas. H.C.I. gratefully acknowledges support in the form of a Research Fellowship for Young Scientists by the Japan Society for the Promotion of Science (JSPS).

\section{References}

Ackermann M, Doebeli M (2004) Evolution of niche width and adaptive diversification. Evolution 58: 2599-2612

Allen CE, Beldade P, Zwaan BJ, Brakefield PM (2008) Differences in the selection response of serially repeated color pattern characters: Standing variation, development, and evolution. BMC Evolutionary Biology 8

Bowers RG, White A, Boots M, Geritz SAH, Kisdi E (2003) Evolutionary branching/speciation: Contrasting results from systems with explicit or emergent carrying capacities. Evolutionary Ecology Research 5:883-891

Bowers RG, Hoyle A, White A, Boots M (2005) The geometric theory of adaptive evolution: Trade-off and invasion plots. Journal of Theoretical Biology 233:363-377

deMazancourt C, Dieckmann U (2004) Trade-off geometries and frequency-dependent selection. American Naturalist 164: 765-778

Dieckmann U, Doebeli M (1999) On the origin of species by sympatric speciation. Nature 400: $354-357$

Dieckmann U, Heino M, Parvinen K (2006) The adaptive dynamics of function-valued traits. Journal of Theoretical Biology 241: 370-389

Dieckmann U, Law R (1996) The dynamical theory of coevolution: A derivation from stochastic ecological processes. Journal of Mathematical Biology 34: 579-612

Dieckmann U, Metz JAJ, Doebeli M, Tautz D (eds) (2004) Adaptive speciation. Cambridge University Press, Cambridge

Durinx M, Metz JAJ, Meszéna G (2008) Adaptive dynamics for physiologically structured population models. Journal of Mathematical Biology 56: 673-742.

Egas M, Sabelis MW, Dieckmann U (2005) Evolution of specialization and ecological character displacement of herbivores along a gradient of plant quality. Evolution 59: $507-520$

Eshel I (1983) Evolutionary and continuous stability. Journal of Theoretical Biology 103: 99-111

Flatt T, Heyland A (eds) (2011). Mechanisms of life history evolution: The genetics and physiology of life history traits and trade-offs. Oxford University Press, Oxford 
Geritz SAH, Kisdi E, Meszéna G, Metz JAJ (1998) Evolutionarily singular strategies and the adaptive growth and branching of the evolutionary tree. Evolutionary Ecology 12: 35-57

Geritz SAH, Kisdi E, Yan P (2007) Evolutionary branching and long-term coexistence of cycling predators: Critical function analysis. Theoretical Population Biology 71: 424435

Geritz SAH, Metz JAJ, Kisdi E, Meszéna G (1997) Dynamics of adaptation and evolutionary branching. Physical Review Letters 78: 2024-2027

Geritz SAH, Metz JAJ, Rueffler C (2016) Mutual invadability near evolutionarily singular strategies for multivariate traits, with special reference to the strongly convergence stable case. Journal of Mathematical Biology 72: 1081-1099

Hall SR, Becker CR, Duffy M, Cceres C (2012) A power-efficiency tradeoff alters epidemiological relationships. Ecology 93: 645-656

Ito HC, Dieckmann U (2007) A new mechanism for recurrent adaptive radiations. The American Naturalist 170: E96-E111

Ito HC, Dieckmann U (2012) Evolutionary-branching lines and areas in bivariate trait spaces. Evolutionary Ecology Research 14: 555-582

Ito HC, Dieckmann U (2014) Evolutionary branching under slow directional evolution. Journal of Theoretical Biology 360: 290-314

Kisdi E (2006) Trade-off geometries and the adaptive dynamics of two co-evolving species. Evolutionary Ecology Research 8: 959-973

Kisdi E (2015) Construction of multiple trade-offs to obtain arbitrary singularities of adaptive dynamics. Journal of Mathematical Biology 70: 1093-1117

Leimar O (2005) The evolution of phenotypic polymorphism: randomized strategies versus evolutionary branching. American Naturalist 165: 669-681

Leimar O (2009) Multidimensional convergence stability. Evolutionary Ecology Research 11: 191-208

Levins R (1962) Theory of fitness in a heterogeneous environment. i. American Naturalist 96: 361-373

Levins R (1968) Evolution in changing environments: some theoretical explorations. Princeton University Press, Princeton, N.J.

Mandy D (2013) On second order conditions for equality constrained extremum problems. Economics Letters 121: 440-443

Maynard Smith J (1982) Evolution and the theory of games. Cambridge University Press

Meszéna G, Gyllenberg M, Pasztor L, Metz JAJ (2006) Competitive exclusion and limiting similarity: A unified theory. Theoretical Population Biology 69: 68-87.

Meszéna G, Metz JAJ (1999) Species diversity and population regulation: the importance of environmental feedback dimensionality. IIASA Working Paper \#WP-99-045 (available at: http://www.iiasa.ac.at/cgi-bin/pubsrch?IR99045). 
Metz JAJ, Geritz SAH, Meszéna G, Jacobs FJA, vanHeerwaarden JS (1996) Adaptive dynamics, a geometrical study of the consequences of nearly faithful reproduction. In: vanStrien SJ, Verduyn-Lunel SM (eds) Stochastic and spatial structures of dynamical systems. North Holland, Amsterdam, The Netherlands, pp 83-231

Metz JAJ, Mylius S, Diekmann O (2008) When does evolution optimize? Evol Ecol Res 10: 629-654

Parvinen K, Heino M, Dieckmann U (2013) Function-valued adaptive dynamics and optimal control theory. Journal of Mathematical Biology 67: 509-533

Rueffler C, van Dooren TJM, Metz JAJ (2004) Adaptive walks on changing landscapes: Levins' approach extended. Theoretical Population Biology 65:165-178.

Schluter D (1996) Adaptive radiation along genetic lines of least resistance. Evolution 50:1766-1774

Vukics A, Asboth J, Meszéna G (2003) Speciation in multidimensional evolutionary space. Physical Review E 68: 041903

\section{Figure Legends}

\section{Figure 1}

Gradient of Lagrange fitness function. In a two-dimensional trait space $\mathbf{s}=(x, y)^{\mathrm{T}}$, a constraint curve $h\left(\mathbf{s}^{\prime}\right)=0$ and its tangent line $\mathbf{s}_{\mathrm{E}}$ at $\mathbf{s}, \mathbf{n} \cdot\left(\mathbf{s}_{\mathrm{E}}-\mathbf{s}\right)=0$ are indicated by the thick solid curve and thin solid straight line, respectively. $\mathbf{n}$ and e are the normal and tangent vectors of the curve at $\mathbf{s}$, respectively. The gradient of the original fitness function, $\mathbf{g}=\nabla_{\mathbf{s}^{\prime}} F(\mathbf{s} ; \mathbf{s})$, and that of its Lagrange fitness function, $\nabla_{\mathbf{s}^{\prime}} F_{\mathrm{L}}\left(\mathbf{s} ; \mathbf{s} ; \lambda_{\mathbf{s}}\right)$, are indicated by thick solid and thick dashed arrows, respectively.

\section{Figure 2}

Effect of curvature of the constraint curve on evolutionary stability for evolutionarily singular points along the curve, when the original fitness landscape has no curvature $(\mathbf{D}=\mathbf{0})$. Grayscale gradations in $(a, b)$ show the fitness landscapes for $\mathbf{s}$, i.e., invasion fitnesses of various mutants $\mathbf{s}^{\prime}$ for a fixed resident $\mathbf{s}, F\left(\mathbf{s}^{\prime} ; \mathbf{s}\right)$ : lighter colors indicate higher fitnesses. In (a), opposite directions between the fitness gradient $\mathbf{g}=\nabla_{\mathbf{s}^{\prime}} F(\mathbf{s} ; \mathbf{s})$ and the curvature vector $\mathbf{q}$ make the fitness landscape along the constraint curve (solid curve) more convex (the apparent fitness curvature $\Omega=\mathbf{g} \cdot \mathbf{q}$ is negative), as illustrated in panel (c). In (b), 
they are in the same direction, which makes the fitness landscape more concave ( $\Omega$ is positive), as illustrated in panel (d).

\section{Figure 3}

Evolutionarily singular points in a two-dimensional resource competition model with a constraint. In the two-dimensional trait spaces $\mathbf{s}=(x, y)^{\mathrm{T}}$, the black curves are the constraint curve (Eq. (25)). The grayscale gradations indicate the carrying capacity distributions, with lighter colors reflecting higher capacities. Dashed curves indicate the contours of the carrying capacities that are tangent to the constraint curves. (a) For $a b<1$, there is only a single evolutionarily singular point $\mathbf{s}_{1}$, which is always convergence stable (filled with black). (b) For $a b>1$, there are two evolutionarily singular points $\mathbf{s}_{1}$ and $\mathbf{s}_{2}$. Point $\mathbf{s}_{2}$ is always convergence stable (filled with black), whereas $\mathbf{s}_{1}$ never is (filled with white). Parameters: $(a, b)=(0.8,1.0)$ for $(a)$, and $(a, b)=(2.5,1.0)$ for $(b)$.

\section{Figure 4}

Parameter dependency on evolutionary branching in example 1. In regions A and $C$, respectively, $\mathbf{s}_{1}$ and $\mathbf{s}_{2}$ are unique convergence stable points, which are evolutionarily unstable (i.e., evolutionary branching points).

\section{Figure 5}

Illustration of the choice of constraint surface, with point $\mathbf{s}$ being a candidate branching point (CBP) along the surface. In a three-dimensional trait space $\mathbf{s}=$ $\left(x_{1}, x_{2}, x_{3}\right)^{\mathrm{T}}$, red surfaces indicate isosurfaces of the carrying capacity distribution $K(\mathbf{s})$, and the blue surface indicates a constraint surface $h\left(\mathbf{s}^{\prime}\right)=0$ on which point $\mathbf{s}$ becomes a CBP (strongly convergence stable and evolutionarily unstable point). 


\section{Appendix A: Proof of Theorem 1}

In the proof we first obtain the projection of $F\left(\mathbf{s}^{\prime} ; \mathbf{s}^{\circ}\right)$ along the constraint curve on its tangent line, which can be treated as a one-dimensional trait space without constraint. This operation corresponds to local parameterization of the constraint curve by using its tangent line as a parameter space. Then we apply the conventional one-dimensional conditions for evolutionary branching points. The proof is as follows.

\section{A.1. Local projection of invasion fitness function}

In an arbitrary two-dimensional trait space, we consider an arbitrary point $\mathbf{s}$ on an arbitrary smooth constraint curve, i.e., $h(\mathbf{s})=0$. To analyze selection pressures on a population located around an arbitrary point $\mathbf{s}=(x, y)^{\mathrm{T}}$, we suppose a mutant $\mathbf{s}_{\mathrm{h}}^{\prime}=\left(x_{\mathrm{h}}^{\prime}, y_{\mathrm{h}}^{\prime}\right)$ and a resident $\mathbf{s}_{\mathrm{h}}^{\circ}=\left(x_{\mathrm{h}}^{\circ}, y_{\mathrm{h}}^{\circ}\right)^{\mathrm{T}}$ close to $\mathbf{s}$, so that $\varepsilon=$ $\max \left\{\left|\mathbf{s}_{\mathrm{h}}^{\prime}-\mathbf{s}\right|,\left|\mathbf{s}_{\mathrm{h}}^{\circ}-\mathbf{s}\right|\right\}$ with $0<\varepsilon \ll 1$. They are both on the constraint curve, satisfying $h\left(\mathbf{s}_{\mathrm{h}}^{\prime}\right)=0$ and $h\left(\mathbf{s}_{\mathrm{h}}^{\circ}\right)=0$. We consider projection of $\mathbf{s}_{\mathrm{h}}^{\prime}$ and $\mathbf{s}_{\mathrm{h}}^{\circ}$ on the tangent line of the constraint curve at $\mathbf{s}$, expressed as

$$
\begin{aligned}
& \mathbf{s}_{\mathrm{E}}^{\prime}=\mathbf{s}+u^{\prime} \mathbf{e}, \\
& \mathbf{s}_{\mathrm{E}}^{\circ}=\mathbf{s}+u^{\circ} \mathbf{e},
\end{aligned}
$$

where $u^{\prime}=\mathbf{e} \cdot\left(\mathbf{s}_{\mathrm{h}}^{\prime}-\mathbf{s}\right), u^{\circ}=\mathbf{e} \cdot\left(\mathbf{s}_{\mathrm{h}}^{\circ}-\mathbf{s}\right)$, and $\mathbf{e}$ is the tangent vector of the curve at $\mathbf{s}$. Then the following lemma holds (see Appendix B for the proof).

\section{Lemma 1}

For a mutant $\mathbf{s}_{\mathrm{h}}^{\prime}$ and a resident $\mathbf{s}_{\mathrm{h}}^{\circ}$ on constraint curve $h\left(\mathbf{s}^{\prime}\right)=0$, and for their projection $\mathbf{s}_{\mathrm{E}}^{\prime}$ and $\mathbf{s}_{\mathrm{E}}^{\circ}$ on its tangent line at $\mathbf{s}$, the invasion fitness of $\mathbf{s}_{\mathrm{h}}^{\prime}$ against $\mathbf{s}_{\mathrm{h}}^{\circ}, F\left(\mathbf{s}_{\mathrm{h}}^{\prime} ; \mathbf{s}_{\mathrm{h}}^{\circ}\right)$, satisfies

$$
F\left(\mathbf{s}_{\mathrm{h}}^{\prime} ; \mathbf{s}_{\mathrm{h}}^{\circ}\right)=F_{\mathrm{L}}\left(\mathbf{s}_{\mathrm{E}}^{\prime} ; \mathbf{s}_{\mathrm{E}}^{\circ} ; \lambda_{\mathrm{s}}\right)+\mathrm{O}\left(\varepsilon^{3}\right),
$$

where $\varepsilon=\max \left\{\left|\mathbf{s}_{\mathrm{h}}^{\prime}-\mathbf{s}\right|,\left|\mathbf{s}_{\mathrm{h}}^{\circ}-\mathbf{s}\right|\right\}$, and

$$
\begin{array}{r}
F_{\mathrm{L}}\left(\mathbf{s}^{\prime} ; \mathbf{s}^{\circ} ; \lambda_{\mathbf{s}}\right)=F\left(\mathbf{s}^{\prime} ; \mathbf{s}^{\circ}\right)-\lambda_{\mathbf{s}}\left[h\left(\mathbf{s}^{\prime}\right)-h\left(\mathbf{s}^{\circ}\right)\right], \\
\lambda_{\mathbf{s}}=\frac{\nabla h(\mathbf{s}) \cdot \nabla_{\mathbf{s}^{\prime}} F(\mathbf{s} ; \mathbf{s})}{|\nabla h(\mathbf{s})|^{2}}=\frac{\mathbf{n} \cdot \mathbf{g}}{|\nabla h(\mathbf{s})|} .
\end{array}
$$

Thus, whether $\mathbf{s}$ is an evolutionary branching point along the constraint curve can be examined by analyzing whether $\mathbf{s}$ is an evolutionary branching point along the tangent line for $F_{\mathrm{L}}\left(\mathbf{s}_{\mathrm{E}}^{\prime} ; \mathbf{s}_{\mathrm{E}}^{\circ} ; \lambda_{\mathbf{s}}\right)$. Since $F_{\mathrm{L}}(\mathbf{s} ; \mathbf{s} ; \lambda)=0$ holds for any $\mathbf{s}$, we can expand $F_{\mathrm{L}}\left(\mathbf{s}_{\mathrm{E}}^{\prime} ; \mathbf{s}_{\mathrm{E}}^{\circ} ; \lambda_{\mathbf{s}}\right)$ at $\mathbf{s}$ as 


$$
\begin{aligned}
F_{\mathrm{L}}\left(\mathbf{s}_{\mathrm{E}}^{\prime} ; \mathbf{s}_{\mathrm{E}}^{\circ} ; \lambda_{\mathbf{s}}\right)=\mathbf{g}_{\mathrm{L}}^{\mathrm{T}}\left(\mathbf{s}_{\mathrm{E}}^{\prime}-\mathbf{s}_{\mathrm{E}}^{\circ}\right)+\frac{1}{2}\left(\mathbf{s}_{\mathrm{E}}^{\prime}-\mathbf{s}_{\mathrm{E}}^{\circ}\right)^{\mathrm{T}} \mathbf{D}_{\mathrm{L}}\left(\mathbf{s}_{\mathrm{E}}^{\prime}-\mathbf{s}_{\mathrm{E}}^{\circ}\right) \\
+\left(\mathbf{s}_{\mathrm{E}}^{\circ}-\mathbf{s}\right)^{\mathrm{T}} \mathbf{C}_{\mathrm{L}}\left(\mathbf{s}_{\mathrm{E}}^{\prime}-\mathbf{s}_{\mathrm{E}}^{\circ}\right)+\mathrm{O}\left(\varepsilon^{3}\right) .
\end{aligned}
$$

with

$$
\begin{aligned}
& \mathbf{g}_{\mathrm{L}}^{\mathrm{T}}=\nabla_{\mathbf{s}^{\prime}}^{\mathrm{T}} F_{\mathrm{L}}\left(\mathbf{s} ; \mathbf{s} ; \lambda_{\mathbf{s}}\right), \\
& \mathbf{D}_{\mathrm{L}}=\nabla_{\mathbf{s}^{\prime}} \nabla_{\mathbf{s}^{\prime}}^{\mathrm{T}} F_{\mathrm{L}}\left(\mathbf{s} ; \mathbf{s} ; \lambda_{\mathbf{s}}\right), \\
& \mathbf{C}_{\mathrm{L}}=\nabla_{\mathbf{s}^{\prime}} \nabla_{\mathbf{s}^{\prime}}^{\mathrm{T}} F_{\mathrm{L}}\left(\mathbf{s} ; \mathbf{s} ; \lambda_{\mathbf{s}}\right)+\nabla_{\mathbf{s}^{\circ}} \nabla_{\mathbf{s}^{\prime}}^{\mathrm{T}} F_{\mathrm{L}}\left(\mathbf{s} ; \mathbf{s} ; \lambda_{\mathbf{s}}\right)
\end{aligned}
$$

(see Ito and Dieckmann (2014) for details of this expansion). By substituting Eqs. (A.1) into Eq. (A.4), we define $F_{\mathrm{U}}\left(u^{\prime} ; u^{\circ}\right)=F_{\mathrm{L}}\left(\mathbf{s}+u^{\prime} \mathbf{e} ; \mathbf{s}+u^{\circ} \mathbf{e} ; \lambda_{\mathbf{s}}\right)$, which is transformed into

$$
\begin{gathered}
F_{\mathrm{U}}\left(u^{\prime} ; u^{\circ}\right)=\mathbf{g}_{\mathrm{L}}^{\mathrm{T}} \mathbf{e}\left(u^{\prime}-u^{\circ}\right)+\frac{1}{2} \mathbf{e}^{\mathrm{T}} \mathbf{D}_{\mathrm{L}} \mathbf{e}\left(u^{\prime}-u^{\circ}\right)^{2} \\
+\mathbf{e}^{\mathrm{T}} \mathbf{C}_{\mathrm{L}} \mathbf{e} u^{\circ}\left(u^{\prime}-u^{\circ}\right)+\mathrm{O}\left(\varepsilon^{3}\right) .
\end{gathered}
$$

Thus, $F_{\mathrm{U}}\left(u^{\prime} ; u^{\circ}\right)$ can be treated as an invasion fitness function of mutant $u^{\prime}$ against resident $u^{\circ}$ in a one-dimensional trait space $u$, where $\mathbf{s}$ corresponds to $u=0$.

\section{A.2. Conditions for evolutionary branching}

To one-dimensional space $u$, we apply the conventional one-dimensional conditions for evolutionary branching (Geritz et.al, 1997), which are (i) evolutionary singularity at $u=0$,

$$
\left[\frac{\partial F_{\mathrm{U}}\left(u^{\prime} ; u^{\circ}\right)}{\partial u^{\prime}}\right]_{u^{\prime}=u^{\circ}=0}=\mathbf{g}_{\mathrm{L}}^{\mathrm{T}} \mathbf{e}=0,
$$

and (ii) its convergence stability, i.e.,

$$
\left[\frac{\partial^{2} F_{\mathrm{U}}\left(u^{\prime} ; u^{\circ}\right)}{\partial u^{\prime 2}}+\frac{\partial^{2} F_{\mathrm{U}}\left(u^{\prime} ; u^{\circ}\right)}{\partial u^{\prime} \partial u^{\circ}}\right]_{u^{\prime}=u^{\circ}=0}=\mathbf{e}^{\mathrm{T}} \mathbf{C}_{\mathrm{L}} \mathbf{e}<0,
$$

and (iii) its evolutionary instability, i.e.,

$$
\left[\frac{\partial^{2} F_{\mathrm{U}}\left(u^{\prime} ; u^{\circ}\right)}{\partial u^{\prime 2}}\right]_{u^{\prime}=u^{\circ}=0}=\mathbf{e}^{\mathrm{T}} \mathbf{D}_{\mathrm{L}} \mathbf{e}>0 .
$$

Because the element of $\mathbf{g}_{\mathrm{L}}$ orthogonal to the constraint curve is always absent (Eq. (8) in the main text), Eq. (A.7) is equivalent to

$$
\mathbf{g}_{\mathrm{L}}=\mathbf{0} \text {. }
$$

Therefore, Eqs. (A.7-9) are identical to Eqs. (10) in Theorem 1, respectively. This completes the proof for Theorem 1. 


\section{A.3. Derivation of Eqs. (9)}

When the constraint curve is described with a parameter $\phi$ as in the main text, i.e., $\mathbf{s}_{\mathrm{h}}^{\circ}=\mathbf{s}\left(\phi^{\circ}\right)=\left(x\left(\phi^{\circ}\right), y\left(\phi^{\circ}\right)\right)^{\mathrm{T}}$ and $\mathbf{s}_{\mathrm{h}}^{\prime}=\mathbf{s}\left(\phi^{\prime}\right)=\left(x\left(\phi^{\prime}\right), y\left(\phi^{\prime}\right)\right)^{\mathrm{T}}$, Lemma 1 is expressed as

$$
F\left(\mathbf{s}\left(\phi^{\prime}\right) ; \mathbf{s}\left(\phi^{\circ}\right)\right)=F_{\mathrm{U}}\left(u^{\prime} ; u^{\circ}\right)+\mathrm{O}\left(\varepsilon^{3}\right),
$$

where $u^{\prime}=\mathbf{e} \cdot\left[\mathbf{s}\left(\phi^{\prime}\right)-\mathbf{s}\right], u^{\circ}=\mathbf{e} \cdot\left[\mathbf{s}\left(\phi^{\circ}\right)-\mathbf{s}\right]$. As in the main text, we assume that the scale of $\phi$ is adjusted so that $|(\mathrm{d} x(\phi) / \mathrm{d} \phi, \mathrm{d} y(\phi) / \mathrm{d} \phi)|=1$ always holds. Then the tangent vector at $\mathbf{s}(\phi)$ is simply given by

$$
\mathbf{e}(\mathbf{s}(\phi))=\frac{\mathrm{d} \mathbf{s}(\phi)}{\mathrm{d} \phi}=\left(\frac{\mathrm{d} x(\phi)}{\mathrm{d} \phi}, \frac{\mathrm{d} y(\phi)}{\mathrm{d} \phi}\right)^{\mathrm{T}}
$$

and thus $\mathbf{e}(\mathbf{s}(\phi))=\mathbf{e}$, which gives

$$
\begin{aligned}
& {\left[\frac{\partial u^{\circ}}{\partial \phi^{\circ}}\right]_{\phi^{\circ}=\phi}=\left[\frac{\partial u^{\prime}}{\partial \phi^{\prime}}\right]_{\phi^{\prime}=\phi}=\left[\frac{\partial \mathbf{e} \cdot \mathbf{s}\left(\phi^{\prime}\right)}{\partial \phi^{\prime}}\right]_{\phi^{\prime}=\phi}=\mathbf{e} \cdot \mathbf{e}=1,} \\
& {\left[\frac{\partial^{2} u^{\circ}}{\partial \phi^{\circ}}\right]_{\phi^{\circ}=\phi}=\left[\frac{\partial^{2} u^{\prime}}{\partial \phi^{\prime 2}}\right]_{\phi^{\prime}=\phi}=\left[\frac{\partial^{2} \mathbf{s}\left(\phi^{\prime}\right)}{\partial \phi^{\prime 2}}\right]_{\phi^{\prime}=\phi}=\frac{1}{2}\left[\frac{\partial \mathbf{e}\left(\mathbf{s}\left(\phi^{\prime}\right)\right) \cdot \mathbf{e}\left(\mathbf{s}\left(\phi^{\prime}\right)\right)}{\partial \phi^{\prime}}\right]_{\phi^{\prime}=\phi}=0,}
\end{aligned}
$$

while $u^{\prime}$ and $u^{\circ}$ are independent of $\phi^{\circ}$ and $\phi^{\prime}$, respectively. Then we see

$$
\begin{aligned}
& {\left[\frac{\partial F\left(\mathbf{s}\left(\phi^{\prime}\right) ; \mathbf{s}\left(\phi^{\circ}\right)\right)}{\partial \phi^{\prime}}\right]_{\phi^{\prime}=\phi^{\circ}=\phi} }=\left[\frac{\partial u^{\prime}}{\partial \phi^{\prime}}\right]_{\phi^{\prime}=\phi^{\circ}=\phi}\left[\frac{\partial F_{\mathrm{U}}\left(u^{\prime} ; u^{\circ}\right)}{\partial u^{\prime}}\right]_{u^{\prime}=u^{\circ}=0} \\
&=\left[\frac{\partial F_{\mathrm{U}}\left(u^{\prime} ; u^{\circ}\right)}{\partial u^{\prime}}\right]_{u^{\prime}=u^{\circ}=0}, \\
& {\left[\frac{\partial^{2} F\left(\mathbf{s}\left(\phi^{\prime}\right) ; \mathbf{s}\left(\phi^{\circ}\right)\right)}{\partial \phi^{\prime 2}}\right]_{\phi^{\prime}=\phi^{\circ}=\phi} }=\left[\frac{\partial^{2} F_{\mathrm{U}}\left(u^{\prime} ; u^{\circ}\right)}{\partial u^{\prime 2}}\right]_{u^{\prime}=u^{\circ}=0}, \\
& {\left[\frac{\partial^{2} F\left(\mathbf{s}\left(\phi^{\prime}\right) ; \mathbf{s}\left(\phi^{\circ}\right)\right)}{\partial \phi^{\circ} \partial \phi^{\prime}}\right]_{\phi^{\prime}=\phi^{\circ}=\phi}=\left[\frac{\partial^{2} F_{\mathrm{U}}\left(u^{\prime} ; u^{\circ}\right)}{\partial u^{\circ} \partial u^{\prime}}\right]_{u^{\prime}=u^{\circ}=0} . }
\end{aligned}
$$

Therefore, by Eqs. (1b), (2c), (2d) in the main text and by Eq. (A.6), we obtain Eqs. (9) in the main text. 


$$
\begin{aligned}
& g(\phi)=\left[\frac{\partial F_{\mathrm{U}}\left(u^{\prime} ; u^{\circ}\right)}{\partial u^{\prime}}\right]_{u^{\prime}=u^{\circ}=0}=\mathbf{g}_{\mathrm{L}}^{\mathrm{T}} \mathbf{e}=\nabla_{\mathbf{s}^{\prime}}^{\mathrm{T}} F_{\mathrm{L}}\left(\mathbf{s} ; \mathbf{s} ; \lambda_{\mathbf{s}}\right) \mathbf{e}, \\
& D(\phi)=\left[\frac{\partial^{2} F_{\mathrm{U}}\left(u^{\prime} ; u^{\circ}\right)}{\partial u^{\prime 2}}\right]_{u^{\prime}=u^{\circ}=0}=\mathbf{e}^{\mathrm{T}} \mathbf{D}_{\mathrm{L}} \mathbf{e} \\
& =\mathbf{e}^{\mathrm{T}} \nabla_{\mathbf{s}^{\prime}} \nabla_{\mathbf{s}^{\mathrm{T}}}^{\mathrm{T}} F_{\mathrm{L}}\left(\mathbf{s} ; \mathbf{s} ; \lambda_{\mathbf{s}}\right) \mathbf{e} \\
& C(\phi)=\left[\frac{\partial^{2} F_{\mathrm{U}}\left(u^{\prime} ; u^{\circ}\right)}{\partial u^{\prime 2}}\right]_{u^{\prime}=u^{\circ}=0}+\left[\frac{\partial^{2} F_{\mathrm{U}}\left(u^{\prime} ; u^{\circ}\right)}{\partial u^{\circ} \partial u^{\prime}}\right]_{u^{\prime}=u^{\circ}=0}=\mathbf{e}^{\mathrm{T}} \mathbf{C}_{\mathrm{L}} \mathbf{e} \\
& =\mathbf{e}^{\mathrm{T}}\left[\left(\nabla_{\mathbf{s}^{\prime}} \nabla_{\mathbf{s}^{\prime}}^{\mathrm{T}}+\nabla_{\mathbf{s}^{\circ}} \nabla_{\mathbf{s}^{\prime}}^{\mathrm{T}}\right) F_{\mathrm{L}}\left(\mathbf{s} ; \mathbf{s} ; \lambda_{\mathbf{s}}\right)\right] \mathbf{e} .
\end{aligned}
$$

\section{Appendix B: Proof of Lemma 1}

Here we prove Lemma 1 . Around an arbitrary point $\mathbf{s}=(x, y)^{\mathrm{T}}$ on the constraint curve expressed by points $\mathbf{s}_{\mathrm{h}}=\left(x_{\mathrm{h}}, y_{\mathrm{h}}\right)^{\mathrm{T}}$ satisfying $h\left(\mathbf{s}_{\mathrm{h}}\right)=0$, we project invasion fitness $F\left(\mathbf{s}_{\mathrm{h}}^{\prime}, \mathbf{s}_{\mathrm{h}}^{\circ}\right)$ on the tangent line of the constraint curve at $\mathbf{s}$. For the projection, we expand $h\left(\mathbf{s}_{\mathrm{h}}\right)$ at $\mathbf{s}$ as

$$
h\left(\mathbf{s}_{\mathrm{h}}\right)=\nabla h(\mathbf{s})^{\mathrm{T}}\left(\mathbf{s}_{\mathrm{h}}-\mathbf{s}\right)+\frac{1}{2}\left(\mathbf{s}_{\mathrm{h}}-\mathbf{s}\right)^{\mathrm{T}} \nabla \nabla^{\mathrm{T}} h(\mathbf{s})\left(\mathbf{s}_{\mathrm{h}}-\mathbf{s}\right)+\mathrm{O}\left(\varepsilon^{3}\right)=0 .
$$

With the normal vector $\mathbf{n}=\left(n_{\mathrm{x}}, n_{\mathrm{y}}\right)^{\mathrm{T}}=\nabla h(\mathbf{s}) /|\nabla h(\mathbf{s})|$ and the tangent vector $\mathbf{e}=\left(n_{\mathrm{y}},-n_{\mathrm{x}}\right)^{\mathrm{T}}$, we express $\mathbf{s}_{\mathrm{h}}$ as

$$
\mathbf{s}_{\mathrm{h}}=\mathbf{e} u+\mathbf{n} w+\mathbf{s}=\left(\begin{array}{ll}
\mathbf{e} & \mathbf{n}
\end{array}\right)\left(\begin{array}{l}
u \\
w
\end{array}\right)+\mathbf{s} .
$$

which upon substitution into Eq. (B.1) gives

$$
\begin{aligned}
& h\left(\mathbf{s}_{\mathrm{h}}\right)=\nabla h(\mathbf{s})^{\mathrm{T}}\left(\begin{array}{ll}
\mathbf{e} & \mathbf{n}
\end{array}\right)\left(\begin{array}{l}
u \\
w
\end{array}\right)+\frac{1}{2}\left(\begin{array}{c}
u \\
w
\end{array}\right)^{\mathrm{T}}\left(\begin{array}{ll}
\mathbf{e} & \mathbf{n}
\end{array}\right)^{\mathrm{T}} \nabla \nabla^{\mathrm{T}} h(\mathbf{s})\left(\begin{array}{ll}
\mathbf{e} & \mathbf{n}
\end{array}\right)\left(\begin{array}{l}
u \\
w
\end{array}\right)+\mathrm{O}\left(\varepsilon^{3}\right) \\
& =|\nabla h(\mathbf{s})| w+\frac{1}{2}\left(\begin{array}{c}
u \\
w
\end{array}\right)^{\mathrm{T}}\left(\begin{array}{ll}
\mathbf{e}^{\mathrm{T}} \nabla \nabla^{\mathrm{T}} h(\mathbf{s}) \mathbf{e} & \mathbf{e}^{\mathrm{T}} \nabla \nabla^{\mathrm{T}} h(\mathbf{s}) \mathbf{n} \\
\mathbf{n}^{\mathrm{T}} \nabla \nabla^{\mathrm{T}} h(\mathbf{s}) \mathbf{e} & \mathbf{n}^{\mathrm{T}} \nabla \nabla^{\mathrm{T}} h(\mathbf{s}) \mathbf{n}
\end{array}\right)\left(\begin{array}{l}
u \\
w
\end{array}\right)+\mathrm{O}\left(\varepsilon^{3}\right)=0 .
\end{aligned}
$$

Solving this equation for $w$ gives

$$
w=-\frac{\mathbf{e}^{\mathrm{T}} \nabla \nabla^{\mathrm{T}} h(\mathbf{s}) \mathbf{e}}{2|\nabla h(\mathbf{s})|} u^{2}+O\left(\varepsilon^{3}\right) .
$$

By introducing the orthogonal projection of $\mathbf{s}_{\mathrm{h}}$ on the tangent line, denoted by $\mathbf{s}_{\mathrm{E}}=\mathbf{e} u+\mathbf{s}=\mathbf{e}\left[\mathbf{e}^{\mathrm{T}}\left(\mathbf{s}_{\mathrm{h}}-\mathbf{s}\right)\right]+\mathbf{s}$, we see 


$$
\begin{aligned}
\mathbf{s}_{\mathrm{h}} & =\mathbf{e} u+\mathbf{s}+\mathbf{n} w=\mathbf{s}_{\mathrm{E}}+\mathbf{n}\left[-\frac{\left(\mathbf{s}_{\mathrm{E}}-\mathbf{s}\right)^{\mathrm{T}} \nabla \nabla^{\mathrm{T}} h(\mathbf{s})\left(\mathbf{s}_{\mathrm{E}}-\mathbf{s}\right)}{2|\nabla h(\mathbf{s})|}+\mathrm{O}\left(\varepsilon^{3}\right)\right] \\
& =\mathbf{s}_{\mathrm{E}}-\frac{\mathbf{n}}{|\nabla h(\mathbf{s})|}\left[\frac{1}{2}\left(\mathbf{s}_{\mathrm{E}}-\mathbf{s}\right)^{\mathrm{T}} \nabla \nabla^{\mathrm{T}} h(\mathbf{s})\left(\mathbf{s}_{\mathrm{E}}-\mathbf{s}\right)+\mathrm{O}\left(\varepsilon^{3}\right)\right] \\
& =\mathbf{s}_{\mathrm{E}}-\frac{\mathbf{n}}{|\nabla h(\mathbf{s})|}\left[h(\mathbf{s})+\nabla^{\mathrm{T}} h(\mathbf{s})\left(\mathbf{s}_{\mathrm{E}}-\mathbf{s}\right)+\frac{1}{2}\left(\mathbf{s}_{\mathrm{E}}-\mathbf{s}\right)^{\mathrm{T}} \nabla \nabla^{\mathrm{T}} h(\mathbf{s})\left(\mathbf{s}_{\mathrm{E}}-\mathbf{s}\right)+\mathrm{O}\left(\varepsilon^{3}\right)\right] \\
& =\mathbf{s}_{\mathrm{E}}-\frac{\mathbf{n}}{|\nabla h(\mathbf{s})|} h\left(\mathbf{s}_{\mathrm{E}}\right)+\mathrm{O}\left(\varepsilon^{3}\right),
\end{aligned}
$$

where $h(\mathbf{s})+\nabla^{\mathrm{T}} h(\mathbf{s})\left(\mathbf{s}_{\mathrm{E}}-\mathbf{s}\right)=0$ is used. Comparing the first and last row gives $\mathbf{n} h\left(\mathbf{s}_{\mathrm{E}}\right) /|\nabla h(\mathbf{s})|=\mathrm{O}\left(\left|\mathbf{s}_{\mathrm{E}}-\mathbf{s}\right|^{2}\right)=\mathrm{O}\left(\varepsilon^{2}\right)$. Then we expand $F\left(\mathbf{s}_{\mathrm{h}}^{\prime} ; \mathbf{s}_{\mathrm{h}}^{\circ}\right)$ at $\mathbf{s}_{\mathrm{h}}^{\prime}=\mathbf{s}_{\mathrm{E}}^{\prime}$ and $\mathbf{s}_{\mathrm{h}}^{\circ}=\mathbf{s}_{\mathrm{E}}^{\circ}$ as

$$
\begin{aligned}
F\left(\mathbf{s}_{\mathrm{h}}^{\prime} ; \mathbf{s}_{\mathrm{h}}^{\circ}\right)= & F\left(\mathbf{s}_{\mathrm{E}}^{\prime}+\left[-\frac{\mathbf{n}}{|\nabla h(\mathbf{s})|} h\left(\mathbf{s}_{\mathrm{E}}^{\prime}\right)+\mathrm{O}\left(\varepsilon^{3}\right)\right] ; \mathbf{s}_{\mathrm{E}}^{\circ}+\left[-\frac{\mathbf{n}}{|\nabla h(\mathbf{s})|} h\left(\mathbf{s}_{\mathrm{E}}^{\circ}\right)+O\left(\varepsilon^{3}\right)\right]\right) \\
= & F\left(\mathbf{s}_{\mathrm{E}}^{\prime} ; \mathbf{s}_{\mathrm{E}}^{\circ}\right)+\nabla_{\mathbf{s}^{\mathrm{T}}}^{\mathrm{T}} F\left(\mathbf{s}_{\mathrm{E}}^{\prime} ; \mathbf{s}_{\mathrm{E}}^{\circ}\right)\left[-\frac{\mathbf{n}}{|\nabla h(\mathbf{s})|} h\left(\mathbf{s}_{\mathrm{E}}^{\prime}\right)+\mathrm{O}\left(\varepsilon^{3}\right)\right] \\
& +\nabla_{\mathbf{s}^{\circ}}^{\mathrm{T}} F\left(\mathbf{s}_{\mathrm{E}}^{\prime} ; \mathbf{s}_{\mathrm{E}}^{\circ}\right)\left[-\frac{\mathbf{n}}{|\nabla h(\mathbf{s})|} h\left(\mathbf{s}_{\mathrm{E}}^{\circ}\right)+\mathrm{O}\left(\varepsilon^{3}\right)\right]+\mathrm{O}\left(\varepsilon^{4}\right) .
\end{aligned}
$$

By using

$$
\begin{aligned}
& \nabla_{\mathbf{s}^{\prime}}^{\mathrm{T}} F\left(\mathbf{s}_{\mathrm{E}}^{\prime} ; \mathbf{s}_{\mathrm{E}}^{\circ}\right)=\nabla_{\mathbf{s}^{\prime}}^{\mathrm{T}} F(\mathbf{s} ; \mathbf{s})+\mathrm{O}(\varepsilon), \\
& \nabla_{\mathbf{s}^{\circ}}^{\mathrm{T}} F\left(\mathbf{s}_{\mathrm{E}}^{\prime} ; \mathbf{s}_{\mathrm{E}}^{\circ}\right)=\nabla_{\mathbf{s}^{\circ}}^{\mathrm{T}} F(\mathbf{s} ; \mathbf{s})+\mathrm{O}(\varepsilon)=-\nabla_{\mathbf{s}^{\prime}}^{\mathrm{T}} F(\mathbf{s} ; \mathbf{s})+\mathrm{O}(\varepsilon),
\end{aligned}
$$

we further transform Eq. (B.6) into

$$
\begin{aligned}
F\left(\mathbf{s}_{\mathrm{h}}^{\prime} ; \mathbf{s}_{\mathrm{h}}^{\circ}\right) & =F\left(\mathbf{s}_{\mathrm{E}}^{\prime} ; \mathbf{s}_{\mathrm{E}}^{\circ}\right)-\nabla_{\mathbf{s}^{\prime}}^{\mathrm{T}} F(\mathbf{s} ; \mathbf{s}) \frac{\mathbf{n}}{|\nabla h(\mathbf{s})|}\left[h\left(\mathbf{s}_{\mathrm{E}}^{\prime}\right)-h\left(\mathbf{s}_{\mathrm{E}}^{\circ}\right)\right]+\mathrm{O}\left(\varepsilon^{3}\right) \\
& =F\left(\mathbf{s}_{\mathrm{E}}^{\prime} ; \mathbf{s}_{\mathrm{E}}^{\circ}\right)-\lambda_{\mathbf{s}}\left[h\left(\mathbf{s}_{\mathrm{E}}^{\prime}\right)-h\left(\mathbf{s}_{\mathrm{E}}^{\circ}\right)\right]+\mathrm{O}\left(\varepsilon^{3}\right)
\end{aligned}
$$

with

$$
\lambda_{\mathbf{s}}=\frac{\nabla_{\mathbf{s}^{\prime}}^{\mathrm{T}} F(\mathbf{s} ; \mathbf{s}) \nabla h(\mathbf{s})}{|\nabla h(\mathbf{s})|^{2}} .
$$

This completes the proof.

\section{Appendix C: Proof of Theorem 2}

In a manner similar to the proof of Theorem 1, we first obtain the projection of $F\left(\mathbf{s}^{\prime} ; \mathbf{s}^{\circ}\right)$ along the constraint surface on its tangent plane. This operation 
corresponds to local parameterization of the constraint surface by using its tangent plane as a parameter space. Then we apply conditions for existence of CBPs (candidate branching points) in multi-dimensional trait spaces without constraint.

\section{C.1. Local projection of invasion fitness function}

In an arbitrary $M$-dimensional trait space, we consider an arbitrary point $\mathbf{s}=$ $\left(x_{1}, \cdots, x_{M}\right)^{\mathrm{T}}$ on an arbitrary $L$-dimensional constraint surface, i.e., $\mathbf{h}(\mathbf{s})=$ $\left(h_{L+1}(\mathbf{s}), \cdots, h_{M}(\mathbf{s})\right)^{\mathrm{T}}=\mathbf{0}$. To analyze selection pressures on a population located around $\mathbf{s}$, we suppose a mutant $\mathbf{s}_{\mathrm{h}}^{\prime}=\left(x_{\mathrm{h} 1}^{\prime}, \cdots, x_{\mathrm{h} M}^{\prime}\right)^{\mathrm{T}}$ and a resident $\mathbf{s}_{\mathrm{h}}^{\circ}=$ $\left(x_{\mathrm{h} 1}^{\circ}, \cdots, x_{\mathrm{h} M}^{\circ}\right)^{\mathrm{T}}$ close to $\mathbf{s}$, so that $\varepsilon=\max \left\{\left|\mathbf{s}_{\mathrm{h}}^{\prime}-\mathbf{s}\right|,\left|\mathbf{s}_{\mathrm{h}}^{\circ}-\mathbf{s}\right|\right\}$ with $0<\varepsilon \ll 1$. They are both on the constraint surface, satisfying $\mathbf{h}\left(\mathbf{s}_{\mathrm{h}}^{\prime}\right)=0$ and $\mathbf{h}\left(\mathbf{s}_{\mathrm{h}}^{\circ}\right)=0$. We consider projection of $\mathbf{s}_{\mathrm{h}}^{\prime}$ and $\mathbf{s}_{\mathrm{h}}^{\circ}$ on the tangent plane of the surface at $\mathbf{s}$, expressed as

$$
\begin{aligned}
& \mathbf{s}_{\mathrm{E}}^{\prime}=\sum_{i=1}^{L} u_{i}^{\prime} \mathbf{e}_{i}+\mathbf{s}=\left(\mathbf{e}_{1}, \cdots, \mathbf{e}_{L}\right)\left(u_{1}^{\prime}, \cdots, u_{L}^{\prime}\right)^{\mathrm{T}}+\mathbf{s}=\mathbf{E} \mathbf{u}^{\prime}+\mathbf{s}, \\
& \mathbf{s}_{\mathrm{E}}^{\circ}=\sum_{i=1}^{L} u_{i}^{\circ} \mathbf{e}_{i}+\mathbf{s}=\left(\mathbf{e}_{1}, \cdots, \mathbf{e}_{L}\right)\left(u_{1}^{\circ}, \cdots, u_{L}^{\circ}\right)^{\mathrm{T}}+\mathbf{s}=\mathbf{E u}^{\circ}+\mathbf{s},
\end{aligned}
$$

where $u_{i}^{\prime}=\left[\mathbf{e}_{i} \cdot\left(\mathbf{s}_{\mathrm{h}}^{\prime}-\mathbf{s}\right)\right]$ and $u_{i}^{\circ}=\left[\mathbf{e}_{i} \cdot\left(\mathbf{s}_{\mathrm{h}}^{\circ}-\mathbf{s}\right)\right]$ with the orthogonal base vectors $\mathbf{e}_{1}, \cdots \mathbf{e}_{L}$ of the surface, satisfying $\left|\mathbf{e}_{i}\right|=1, \mathbf{e}_{i} \cdot \mathbf{e}_{i^{\prime}}=0$, and $\mathbf{e}_{i} \cdot \mathbf{n}_{j}=0$ for all $j=L+1, \cdots, M$ and $i, i^{\prime}=1, \cdots, L$ with $i \neq i^{\prime}$, where $\mathbf{n}_{L+1}, \cdots, \mathbf{n}_{M}$ are the normal vectors of the surface, given by $\mathbf{n}_{j}=\nabla h_{j}(\mathbf{s}) /\left[\nabla h_{j}(\mathbf{s})\right]$. Then analogously to Lemma 1, the following lemma holds (see Appendix D for the proof).

\section{Lemma 2}

For mutants $\mathbf{s}_{\mathrm{h}}^{\prime}$ and residents $\mathbf{s}_{\mathrm{h}}^{\circ}$ on constraint surface $\mathbf{h}\left(\mathbf{s}^{\prime}\right)=0$, and for their projection $\mathbf{s}_{\mathrm{E}}^{\prime}$ and $\mathbf{s}_{\mathrm{E}}^{\circ}$ on its tangent plane at $\mathbf{s}$, the invasion fitness of $\mathbf{s}_{\mathrm{h}}^{\prime}$ against $\mathbf{s}_{\mathrm{h}}^{\circ}, F\left(\mathbf{s}_{\mathrm{h}}^{\prime} ; \mathbf{s}_{\mathrm{h}}^{\circ}\right)$, satisfies

$$
F\left(\mathbf{s}_{\mathrm{h}}^{\prime} ; \mathbf{s}_{\mathrm{h}}^{\circ}\right)=F_{\mathrm{L}}\left(\mathbf{s}_{\mathrm{E}}^{\prime} ; \mathbf{s}_{\mathrm{E}}^{\circ}, \boldsymbol{\lambda}_{\mathbf{s}}\right)+\mathrm{O}\left(\varepsilon^{3}\right),
$$

where $\varepsilon=\max \left\{\left|\mathbf{s}_{\mathrm{h}}^{\prime}-\mathbf{s}\right|,\left|\mathbf{s}_{\mathrm{h}}^{\circ}-\mathbf{s}\right|\right\}$, and

$$
\begin{aligned}
F_{\mathrm{L}}\left(\mathbf{s}^{\prime} ; \mathbf{s}^{\circ} ; \boldsymbol{\lambda}_{\mathbf{s}}\right) & =F\left(\mathbf{s}^{\prime} ; \mathbf{s}^{\circ}\right)+\lambda_{\mathbf{s}}^{\mathrm{T}}\left[\mathbf{h}\left(\mathbf{s}^{\prime}\right)-\mathbf{h}\left(\mathbf{s}^{\circ}\right)\right], \\
\boldsymbol{\lambda}_{\mathbf{s}} & =\mathbf{B}^{+} \nabla_{\mathbf{s}^{\prime}} F(\mathbf{s} ; \mathbf{s}),
\end{aligned}
$$

where $\mathbf{B}^{+}=\left[\mathbf{B}^{\mathrm{T}} \mathbf{B}\right]^{-1} \mathbf{B}^{\mathrm{T}}$ is the pseudo inverse of the $M$-by- $(M-L)$ matrix $\mathbf{B}=\left(\nabla h_{L+1}(\mathbf{s}), \cdots, \nabla h_{M}(\mathbf{s})\right)$, i.e., $\mathbf{B}^{+} \mathbf{B}$ gives the identity matrix of size $(M-L)$. 
Thus, whether $\mathbf{s}$ is a CBP along the constraint surface can be examined by analyzing whether $\mathbf{s}$ is a CBP along the tangent plane at $\mathbf{s}$ in terms of $F_{\mathrm{L}}\left(\mathbf{s}_{\mathrm{E}}^{\prime} ; \mathbf{s}_{\mathrm{E}}^{\circ} ; \boldsymbol{\lambda}_{\mathbf{s}}\right)$. In the same manner with the two-dimensional case, we expand $F_{\mathrm{L}}\left(\mathbf{s}^{\prime} ; \mathbf{s}^{\circ} ; \boldsymbol{\lambda}_{\mathbf{s}}\right)$ at $\mathbf{s}$ as

$$
\begin{gathered}
F_{\mathrm{L}}\left(\mathbf{s}^{\prime} ; \mathbf{s}^{\circ} ; \boldsymbol{\lambda}_{\mathbf{s}}\right)=\mathbf{g}_{\mathrm{L}}^{\mathrm{T}}\left(\mathbf{s}^{\prime}-\mathbf{s}^{\circ}\right)+\frac{1}{2}\left(\mathbf{s}^{\prime}-\mathbf{s}^{\circ}\right)^{\mathrm{T}} \mathbf{D}_{\mathrm{L}}\left(\mathbf{s}^{\prime}-\mathbf{s}^{\circ}\right) \\
+\left(\mathbf{s}^{\circ}-\mathbf{s}\right)^{\mathrm{T}} \mathbf{C}_{\mathrm{L}}\left(\mathbf{s}^{\prime}-\mathbf{s}^{\circ}\right)+\mathrm{O}\left(\varepsilon^{3}\right)
\end{gathered}
$$

with

$$
\begin{aligned}
& \mathbf{g}_{\mathrm{L}}^{\mathrm{T}}=\nabla_{\mathbf{s}^{\prime}}^{\mathrm{T}} F_{\mathrm{L}}\left(\mathbf{s} ; \mathbf{s} ; \boldsymbol{\lambda}_{\mathbf{s}}\right), \\
& \mathbf{D}_{\mathrm{L}}=\nabla_{\mathbf{s}^{\prime}} \nabla_{\mathbf{s}^{\prime}}^{\mathrm{T}} F_{\mathrm{L}}\left(\mathbf{s} ; \mathbf{s} ; \boldsymbol{\lambda}_{\mathbf{s}}\right), \\
& \mathbf{C}_{\mathrm{L}}=\nabla_{\mathbf{s}^{\prime}} \nabla_{\mathbf{s}^{\prime}}^{\mathrm{T}} F_{\mathrm{L}}\left(\mathbf{s} ; \mathbf{s} ; \boldsymbol{\lambda}_{\mathbf{s}}\right)+\nabla_{\mathbf{s}^{\circ}} \nabla_{\mathbf{s}^{\prime}}^{\mathrm{T}} F_{\mathrm{L}}\left(\mathbf{s} ; \mathbf{s} ; \boldsymbol{\lambda}_{\mathbf{s}}\right) .
\end{aligned}
$$

By substituting Eqs. (C.1) into Eq. (C.4), We define $F_{\mathrm{U}}\left(\mathbf{u}^{\prime} ; \mathbf{u}^{\circ}\right)=F_{\mathrm{L}}\left(\mathbf{E \mathbf { u } ^ { \prime }}+\mathbf{s} ; \mathbf{E u}^{\circ}+\right.$ $\left.\mathbf{s} ; \boldsymbol{\lambda}_{\mathbf{s}}\right)$, which is transformed into

$$
\begin{gathered}
F_{\mathrm{U}}\left(\mathbf{u}^{\prime} ; \mathbf{u}^{\circ}\right)=\mathbf{g}_{\mathrm{L}}^{\mathrm{T}} \mathbf{E}\left(\mathbf{u}^{\prime}-\mathbf{u}^{\circ}\right)+\frac{1}{2}\left(\mathbf{u}^{\prime}-\mathbf{u}^{\circ}\right)^{\mathrm{T}} \mathbf{E}^{\mathrm{T}} \mathbf{D}_{\mathrm{L}} \mathbf{E}\left(\mathbf{u}^{\prime}-\mathbf{u}^{\circ}\right) \\
+\mathbf{u}^{\circ \mathrm{T}} \mathbf{E}^{\mathrm{T}} \mathbf{C}_{\mathrm{L}} \mathbf{E}\left(\mathbf{u}^{\prime}-\mathbf{u}^{\circ}\right)+\mathrm{O}\left(\varepsilon^{3}\right),
\end{gathered}
$$

Thus, $F_{\mathrm{U}}\left(\mathbf{u}^{\prime} ; \mathbf{u}^{\circ}\right)$ can be treated as an invasion fitness function of mutant $\mathbf{u}^{\prime}$ against resident $\mathbf{u}^{\circ}$, in the $L$-dimensional trait space $\mathbf{u}$, where $\mathbf{s}$ corresponds to the origin $\mathbf{u}=\mathbf{0}$.

\section{C.2. Conditions for existence of CBPs}

In an arbitrary M-dimensional trait space $\mathbf{s}=\left(x_{1}, \cdots, x_{M}\right)^{\mathrm{T}}$ with no evolutionary constraint (mutation is possible in all directions), a point $\mathbf{s}$ is a CBP (candidate branching point) when $\mathbf{s}$ satisfies the following three conditions (Ito and Dieckmann, 2012, 2014; Geritz et al. 2016):

(i) $\mathbf{s}$ is evolutionarily singular, satisfying

$$
\nabla_{\mathbf{s}^{\prime}} F(\mathbf{s} ; \mathbf{s})=\mathbf{g}=\mathbf{0} .
$$

(ii) $\mathbf{s}$ is strongly convergence stable (Leimar, 2005), i.e., the symmetric part of an $M-b y-M$ matrix

$$
\left(\nabla_{\mathbf{s}^{\prime}} \nabla_{\mathbf{s}^{\prime}}^{\mathrm{T}}+\nabla_{\mathbf{s}^{\circ}} \nabla_{\mathbf{s}^{\prime}}^{\mathrm{T}}\right) F(\mathbf{s} ; \mathbf{s})=\mathbf{C}
$$

is negative definite.

(iii) $\mathbf{s}$ is evolutionarily unstable, i.e., a symmetric $M-b y-M$ matrix

$$
\nabla_{\mathbf{s}^{\prime}} \nabla_{\mathbf{s}^{\prime}} F(\mathbf{s} ; \mathbf{s})=\mathbf{D}
$$

has at least one positive eigenvalue. 
Here, condition (i) means vanishment of the fitness gradient for resident located at s, while condition (iii) means that the fitness landscape is concave along the eigenvector of the positive eigenvalue, allowing invasion against resident $\mathbf{s}^{\circ}=\mathbf{s}$ by mutants deviated to that direction. Condition (ii) means convergence stability under any genetic correlation among directions (Leimar, 2005, 2009). More specifically, expected directional evolution of a monomorphic unstructured population located close to an evolutionary singular point $\mathbf{s}$ can be described as shift of the monomorphic population's resident phenotype $\mathbf{s}^{\circ}$,

$$
\begin{aligned}
\frac{\mathrm{d} \mathbf{s}^{\circ}}{\mathrm{d} t} & =\frac{n^{\circ} \mu}{2} \mathbf{V}\left(\mathbf{s}^{\circ}\right) \mathbf{g}\left(\mathbf{s}^{\circ}\right) \\
& =\frac{n^{\circ} \mu}{2} \mathbf{V}\left(\mathbf{s}^{\circ}\right) \mathbf{C}^{\mathrm{T}}\left(\mathbf{s}^{\circ}-\mathbf{s}\right)+\text { h. o.t. }
\end{aligned}
$$

with mutational variance-covariance matrix $\mathbf{V}\left(\mathbf{s}^{\circ}\right)$ having $M$ positive eigenvalues that give magnitudes of mutational steps in directions of the corresponding eigenvectors (Dieckmann and Law, 1996). Leimar (2005) has proved that when $\mathbf{C}$ has negative definite symmetric part, then $\mathbf{S}$ is a point attractor for Eq. (C.10) irrespective of $\mathbf{V}\left(\mathbf{s}^{\circ}\right)$, called 'strongly convergence stable.'

On the basis of conditions (i-iii), we derive conditions for $\mathbf{s}$ being a CBP along the constraint surface, as follows. By treating the tangent plane as an $L$ dimensional trait space $\mathbf{u}$ without constraint, from Eq. (C.6) we have

$$
\begin{gathered}
\mathbf{g}_{\mathrm{U}}=\nabla_{\mathbf{u}^{\prime}}^{\mathrm{T}} F_{\mathrm{U}}(\mathbf{0} ; \mathbf{0})=\mathbf{g}_{\mathrm{L}}^{\mathrm{T}} \mathbf{E}, \\
\mathbf{C}_{\mathrm{U}}=\nabla_{\mathbf{u}^{\prime}} \nabla_{\mathbf{u}^{\prime}}^{\mathrm{T}} F_{\mathrm{U}}(\mathbf{0} ; \mathbf{0})+\nabla_{\mathbf{u}^{\circ}} \nabla_{\mathbf{u}^{\mathrm{T}}}^{\mathrm{T}} F_{\mathrm{U}}(\mathbf{0} ; \mathbf{0})=\mathbf{E}^{\mathrm{T}} \mathbf{C}_{\mathrm{L}} \mathbf{E}, \\
\mathbf{D}_{\mathrm{U}}=\nabla_{\mathbf{u}^{\prime}} \nabla_{\mathbf{u}^{\prime}}^{\mathrm{T}} F_{\mathrm{U}}(\mathbf{0} ; \mathbf{0})=\mathbf{E}^{\mathrm{T}} \mathbf{D}_{\mathrm{L}} \mathbf{E},
\end{gathered}
$$

Replacing Eqs. (C.7-9) with $\mathbf{g}_{\mathrm{U}}=\mathbf{0}, \mathbf{C}_{\mathrm{U}}$, and $\mathbf{D}_{\mathrm{U}}$, respectively, we find conditions (ii) and (iii) in Theorem 2. As for condition (i), i.e.,

$$
\mathbf{g}_{\mathrm{U}}=\mathbf{g}_{\mathrm{L}}^{\mathrm{T}} \mathbf{E}=\mathbf{0} \text {, }
$$

the element of $\mathbf{g}_{\mathrm{L}}$ orthogonal to the constraint surface is always absent, because

$$
\begin{aligned}
\mathbf{B}^{\mathrm{T}} \mathbf{g}_{\mathrm{L}} & =\mathbf{B}^{\mathrm{T}}\left[\nabla_{\mathbf{s}^{\prime}} F(\mathbf{s} ; \mathbf{s})-\mathbf{B} \boldsymbol{\lambda}_{\mathbf{s}}\right] \\
& =\mathbf{B}^{\mathrm{T}}\left[\mathbf{g}-\mathbf{B B}^{+} \mathbf{g}\right] \\
& =\mathbf{B}^{\mathrm{T}}\left[\mathbf{I}-\mathbf{B}\left[\mathbf{B}^{\mathrm{T}} \mathbf{B}\right]^{-1} \mathbf{B}^{\mathrm{T}}\right] \mathbf{g} \\
& =\left[\mathbf{B}^{\mathrm{T}}-\left[\mathbf{B}^{\mathrm{T}} \mathbf{B}\right]\left[\mathbf{B}^{\mathrm{T}} \mathbf{B}\right]^{-1} \mathbf{B}^{\mathrm{T}}\right] \mathbf{g} \\
& =\mathbf{0}
\end{aligned}
$$

for $\mathbf{B}=\left(\nabla h_{L+1}(\mathbf{s}), \ldots, \nabla h_{M}(\mathbf{s})\right)$, where $\mathbf{B}^{+}=\left[\mathbf{B}^{\mathrm{T}} \mathbf{B}\right]^{-1} \mathbf{B}^{\mathrm{T}}$ is used. Thus, Eq. (C.14) is equivalent to 


$$
\mathbf{g}_{\mathrm{L}}=\sum_{i=1}^{L}\left[\mathbf{e}_{i} \cdot \mathbf{g}\right] \mathbf{e}_{i}=\mathbf{0},
$$

which gives condition (i) in Theorem 2. This completes the proof for Theorem 2.

\section{Appendix D: Proof of Lemma 2}

Here we prove Lemma 2 in a manner similar to the proof of Lemma 1 (Appendix B). Around an arbitrary point $\mathbf{s}=(x, y)^{\mathrm{T}}$ on the constraint surface expressed by points $\mathbf{s}_{\mathrm{h}}=\left(x_{\mathrm{h}}, y_{\mathrm{h}}\right)^{\mathrm{T}}$ satisfying $\mathbf{h}\left(\mathbf{s}_{\mathrm{h}}\right)=\left(h_{L+1}\left(\mathbf{s}_{\mathrm{h}}\right), \ldots, h_{M}\left(\mathbf{s}_{\mathrm{h}}\right)\right)^{\mathrm{T}}=\mathbf{0}$, we project invasion fitness $F\left(\mathbf{s}_{\mathrm{h}}^{\prime}, \mathbf{s}_{\mathrm{h}}^{\circ}\right)$ on the tangent plane of the constraint surface at $\mathbf{s}$. For the projection, we first expand $h_{j}\left(\mathbf{s}_{\mathrm{h}}\right)$ at $\mathbf{s}$ as

$$
h_{j}\left(\mathbf{s}_{\mathrm{h}}\right)=\nabla^{\mathrm{T}} h_{L+1}(\mathbf{s})\left[\mathbf{s}_{\mathrm{h}}-\mathbf{s}\right]+\frac{1}{2}\left[\mathbf{s}_{\mathrm{h}}-\mathbf{s}\right]^{\mathrm{T}} \nabla \nabla^{\mathrm{T}} h_{L+1}(\mathbf{s})\left[\mathbf{s}_{\mathrm{h}}-\mathbf{s}\right]+\mathrm{O}\left(\varepsilon^{3}\right),
$$

where $\varepsilon=\left|\mathbf{s}_{\mathrm{h}}-\mathbf{s}\right|$. Combining Eq. (D.1) for all $j=L+1, \cdots, M$, we get

$$
\begin{aligned}
\mathbf{h}\left(\mathbf{s}_{\mathrm{h}}\right)=\left(\begin{array}{c}
h_{L+1}\left(\mathbf{s}_{\mathrm{h}}\right) \\
\vdots \\
h_{M}\left(\mathbf{s}_{\mathrm{h}}\right)
\end{array}\right) & =\left(\begin{array}{c}
\nabla^{\mathrm{T}} h_{L+1}(\mathbf{s})\left[\mathbf{s}_{\mathrm{h}}-\mathbf{s}\right]+\frac{1}{2}\left[\mathbf{s}_{\mathrm{h}}-\mathbf{s}\right]^{\mathrm{T}} \nabla \nabla^{\mathrm{T}} h_{L+1}(\mathbf{s})\left[\mathbf{s}_{\mathrm{h}}-\mathbf{s}\right]+\mathrm{O}\left(\varepsilon^{3}\right) \\
\vdots \\
\nabla^{\mathrm{T}} h_{M}(\mathbf{s})\left[\mathbf{s}_{\mathrm{h}}-\mathbf{s}\right]+\frac{1}{2}\left[\mathbf{s}_{\mathrm{h}}-\mathbf{s}\right]^{\mathrm{T}} \nabla \nabla^{\mathrm{T}} h_{M}(\mathbf{s})\left[\mathbf{s}_{\mathrm{h}}-\mathbf{s}\right]+\mathrm{O}\left(\varepsilon^{3}\right)
\end{array}\right) \\
& =\left(\begin{array}{c}
\nabla^{\mathrm{T}} h_{L+1}(\mathbf{s})\left[\mathbf{s}_{\mathrm{h}}-\mathbf{s}\right] \\
\vdots \\
\nabla^{\mathrm{T}} h_{M}(\mathbf{s})\left[\mathbf{s}_{\mathrm{h}}-\mathbf{s}\right]
\end{array}\right)+\frac{1}{2}\left(\begin{array}{c}
{\left[\mathbf{s}_{\mathrm{h}}-\mathbf{s}\right]^{\mathrm{T}} \nabla \nabla^{\mathrm{T}} h_{L+1}(\mathbf{s})\left[\mathbf{s}_{\mathrm{h}}-\mathbf{s}\right]} \\
\vdots \\
{\left[\mathbf{s}_{\mathrm{h}}-\mathbf{s}\right]^{\mathrm{T}} \nabla \nabla^{\mathrm{T}} h_{M}(\mathbf{s})\left[\mathbf{s}_{\mathrm{h}}-\mathbf{s}\right]}
\end{array}\right)+\left(\begin{array}{c}
\mathrm{O}\left(\varepsilon^{3}\right) \\
\vdots \\
0\left(\varepsilon^{3}\right)
\end{array}\right) \\
& =\mathbf{B}^{\mathrm{T}}\left[\mathbf{s}_{\mathrm{h}}-\mathbf{s}\right]+\frac{1}{2}\left[\mathbf{s}_{\mathrm{h}}-\mathbf{s}\right]^{\mathrm{T}} \mathrm{H}\left[\mathbf{s}_{\mathrm{h}}-\mathbf{s}\right]+\mathbf{O}_{M-L}\left(\varepsilon^{3}\right),
\end{aligned}
$$

where $\mathbf{B}^{\mathrm{T}}=\nabla^{\mathrm{T}} \mathbf{h}(\mathbf{s})=\left(\nabla h_{L+1}(\mathbf{s}), \cdots, \nabla h_{M}(\mathbf{s})\right)^{\mathrm{T}}, \mathbf{O}_{M-L}\left(\varepsilon^{3}\right)=\left(\mathrm{O}\left(\varepsilon^{3}\right), \cdots, \mathrm{O}\left(\varepsilon^{3}\right)\right)^{\mathrm{T}}$ of dimension $M-L$, and $\mathrm{H}$ is a column vector having $M$-by- $M$ matrices as its components, given by

$$
\mathrm{H}=\left\{\begin{array}{c}
\mathbf{H}_{L+1} \\
\vdots \\
\mathbf{H}_{M}
\end{array}\right\}=\left\{\begin{array}{c}
\nabla \nabla^{\mathrm{T}} h_{L+1}(\mathbf{s}) \\
\vdots \\
\nabla \nabla^{\mathrm{T}} h_{M}(\mathbf{s})
\end{array}\right\} .
$$

To allow transformation of the second row to the third in Eqs. (D.2), we define multiplication of this kind of vector by usual vectors, and that by matrices, as 


$$
\begin{aligned}
& \mathbf{s}^{\mathrm{T}} \mathrm{H}=\left(\begin{array}{c}
\mathbf{s}^{\mathrm{T}} \mathbf{H}_{L+1} \\
\vdots \\
\mathbf{s}^{\mathrm{T}} \mathbf{H}_{M}
\end{array}\right), \quad \mathbf{s}^{\mathrm{T}} \mathrm{H} \mathbf{s}=\left(\begin{array}{c}
\mathbf{s}^{\mathrm{T}} \mathbf{H}_{L+1} \mathbf{s} \\
\vdots \\
\mathbf{s}^{\mathrm{T}} \mathbf{H}_{M} \mathbf{s}
\end{array}\right), \\
& \mathbf{A}^{\mathrm{T}} \mathbf{H}=\left\{\begin{array}{c}
\mathbf{A}^{\mathrm{T}} \mathbf{H}_{L+1} \\
\vdots \\
\mathbf{A}^{\mathrm{T}} \mathbf{H}_{M}
\end{array}\right\}, \quad \mathbf{H A}=\left\{\begin{array}{c}
\mathbf{H}_{L+1} \mathbf{A} \\
\vdots \\
\mathbf{H}_{M} \mathbf{A}
\end{array}\right\},
\end{aligned}
$$

where $\mathbf{A}$ is an arbitrary $M$-by- $K_{A}$ matrix with arbitrary column length $K_{A}$. With the non-scaled normal vectors $\mathbf{B}=\nabla \mathbf{h}^{\mathrm{T}}(\mathbf{s})=\left[\nabla^{\mathrm{T}} \mathbf{h}(\mathbf{s})\right]^{\mathrm{T}}$ and the tangent vector $\mathbf{E}=\left(\mathbf{e}_{1}, \cdots, \mathbf{e}_{L}\right)$, we express $\mathbf{s}_{\mathrm{h}}$ as

$$
\mathbf{s}_{\mathrm{h}}=\mathbf{E u}+\mathbf{B w}+\mathbf{s}=\left(\begin{array}{ll}
\mathbf{E} & \mathbf{B}
\end{array}\right)\left(\begin{array}{c}
\mathbf{u} \\
\mathbf{w}
\end{array}\right)+\mathbf{s},
$$

where $\mathbf{u}=\mathbf{E}^{\mathrm{T}}\left[\mathbf{s}_{\mathrm{h}}-\mathbf{s}\right]$ and $\mathbf{w}=\left[\mathbf{B}^{\mathrm{T}} \mathbf{B}\right]^{-\mathbf{1}} \mathbf{B}\left[\mathbf{s}_{\mathrm{h}}-\mathbf{s}\right]=\mathbf{B}^{+}\left[\mathbf{s}_{\mathrm{h}}-\mathbf{s}\right]$ because $\left(\begin{array}{ll}\mathbf{E} & \mathbf{B}\end{array}\right)^{-1}=\left(\begin{array}{l}\mathbf{E}^{\mathrm{T}} \\ \mathbf{B}^{+}\end{array}\right)$. Substituting Eq. (D.5) into Eq. (D.2) gives

$$
\begin{aligned}
\mathbf{h}\left(\mathbf{s}_{\mathrm{h}}\right) & =\mathbf{B}^{\mathrm{T}}\left(\begin{array}{ll}
\mathbf{E} & \mathbf{B}
\end{array}\right)\left(\begin{array}{l}
\mathbf{u} \\
\mathbf{w}
\end{array}\right)+\frac{1}{2}\left(\begin{array}{l}
\mathbf{u} \\
\mathbf{w}
\end{array}\right)^{\mathrm{T}}\left(\begin{array}{ll}
\mathbf{E} & \mathbf{B}
\end{array}\right)^{\mathrm{T}} \mathrm{H}\left(\begin{array}{ll}
\mathbf{E} & \mathbf{B}
\end{array}\right)\left(\begin{array}{l}
\mathbf{u} \\
\mathbf{w}
\end{array}\right)+\mathbf{O}_{M-L}\left(\varepsilon^{3}\right) . \\
& =\mathbf{B}^{\mathrm{T}} \mathbf{B} \mathbf{w}+\frac{1}{2}\left(\begin{array}{l}
\mathbf{u} \\
\mathbf{w}
\end{array}\right)^{\mathrm{T}}\left(\begin{array}{cc}
\mathbf{E}^{\mathrm{T}} H \mathbf{E} & \mathbf{E}^{\mathrm{T}} H \mathbf{B} \\
\mathbf{B}^{\mathrm{T}} H \mathbf{E} & \mathbf{B}^{\mathrm{T}} H \mathbf{B}
\end{array}\right)\left(\begin{array}{l}
\mathbf{u} \\
\mathbf{w}
\end{array}\right)+\mathbf{O}_{M-L}\left(\varepsilon^{3}\right)=0 .
\end{aligned}
$$

Solving this equation for $\mathbf{w}$ gives

$$
\mathbf{w}=-\frac{1}{2}\left[\mathbf{B}^{\mathrm{T}} \mathbf{B}\right]^{-1} \mathbf{u}^{\mathrm{T}} \mathbf{E}^{\mathrm{T}} \mathbf{H E u}+\mathbf{O}_{M-L}\left(\varepsilon^{3}\right) .
$$

By introducing $\mathbf{s}_{\mathrm{E}}=\mathbf{E} \mathbf{u}+\mathbf{s}=\mathbf{E}\left[\mathbf{E}^{\mathrm{T}}\left(\mathbf{s}_{\mathrm{h}}-\mathbf{s}\right)\right]+\mathbf{s}$, we transform $\mathbf{s}_{\mathrm{h}}$ as

$$
\begin{aligned}
\mathbf{s}_{\mathrm{h}} & =\mathbf{E} \mathbf{u}+\mathbf{s}+\mathbf{B w}=\mathbf{s}_{\mathrm{E}}+\mathbf{B}\left[-\frac{1}{2}\left[\mathbf{B}^{\mathrm{T}} \mathbf{B}\right]^{-1}\left(\mathbf{s}_{\mathrm{E}}-\mathbf{s}\right)^{\mathrm{T}} \mathrm{H}\left(\mathbf{s}_{\mathrm{E}}-\mathbf{s}\right)+\mathbf{O}_{M-L}\left(\varepsilon^{3}\right)\right] \\
& =\mathbf{s}_{\mathrm{E}}-\mathbf{B}\left[\mathbf{B}^{\mathrm{T}} \mathbf{B}\right]^{-1}\left[\mathbf{h}(\mathbf{s})+\mathbf{B}^{\mathrm{T}}\left(\mathbf{s}_{\mathrm{E}}-\mathbf{s}\right)+\frac{1}{2}\left(\mathbf{s}_{\mathrm{E}}-\mathbf{s}\right)^{\mathrm{T}} \mathrm{H}\left(\mathbf{s}_{\mathrm{E}}-\mathbf{s}\right)+\mathbf{o}_{M-L}\left(\varepsilon^{3}\right)\right] \\
& =\mathbf{s}_{\mathrm{E}}-\mathbf{B}^{+\mathrm{T}} \mathbf{h}\left(\mathbf{s}_{\mathrm{E}}\right)+\mathbf{o}_{M-L}\left(\varepsilon^{3}\right),
\end{aligned}
$$

where $\mathbf{B}^{\mathrm{T}}\left(\mathbf{s}_{\mathrm{E}}-\mathbf{s}\right)=\mathbf{0}$ and $\mathbf{B}^{+\mathrm{T}}=\mathbf{B}\left[\mathbf{B}^{\mathrm{T}} \mathbf{B}\right]^{-1}$. Comparing the first and second row in Eq. (D.8) gives $\mathbf{B}^{+\mathrm{T}} \mathbf{h}\left(\mathbf{s}_{\mathrm{E}}\right)=\mathbf{0}_{M}\left(\left|\mathbf{s}_{\mathrm{E}}\right|^{2}\right)=\mathbf{0}_{M}\left(\varepsilon^{2}\right)$. Then we expand $F\left(\mathbf{s}_{\mathrm{h}}^{\prime} ; \mathbf{s}_{\mathrm{h}}^{\circ}\right)$ at $\mathbf{s}_{\mathrm{h}}^{\prime}=\mathbf{s}_{\mathrm{E}}^{\prime}$ and $\mathbf{s}_{\mathrm{h}}^{\circ}=\mathbf{s}_{\mathrm{E}}^{\circ}$ as

$$
\begin{aligned}
F\left(\mathbf{s}_{\mathrm{h}}^{\prime} ; \mathbf{s}_{\mathrm{h}}^{\circ}\right)= & F\left(\mathbf{s}_{\mathrm{E}}^{\prime}-\mathbf{B}^{+\mathrm{T}} \mathbf{h}\left(\mathbf{s}_{\mathrm{E}}^{\prime}\right)+\mathbf{o}_{M-L}\left(\varepsilon^{3}\right) ; \mathbf{s}_{\mathrm{E}}^{\circ}-\mathbf{B}^{+\mathrm{T}} \mathbf{h}\left(\mathbf{s}_{\mathrm{E}}^{\circ}\right)+\mathbf{o}_{M-L}\left(\varepsilon^{3}\right)\right) \\
= & F\left(\mathbf{s}_{\mathrm{E}}^{\prime} ; \mathbf{s}_{\mathrm{E}}^{\circ}\right)-\nabla_{\mathbf{s}^{\prime}}^{\mathrm{T}} F\left(\mathbf{s}_{\mathrm{E}}^{\prime} ; \mathbf{s}_{\mathrm{E}}^{\circ}\right) \mathbf{B}^{+\mathrm{T}}\left[\mathbf{h}\left(\mathbf{s}_{\mathrm{E}}^{\prime}\right)+\mathbf{o}_{M-L}\left(\varepsilon^{3}\right)\right] \\
& -\nabla_{\mathbf{s}^{\circ}}^{\mathrm{T}} F\left(\mathbf{s}_{\mathrm{E}}^{\prime} ; \mathbf{s}_{\mathrm{E}}^{\circ}\right) \mathbf{B}^{+\mathrm{T}}\left[\mathbf{h}\left(\mathbf{s}_{\mathrm{E}}^{\circ}\right)+\mathbf{o}_{M-L}\left(\varepsilon^{3}\right)\right]+O\left(\varepsilon^{4}\right) .
\end{aligned}
$$

By using 


$$
\begin{aligned}
& \nabla_{\mathbf{s}^{\prime}}^{\mathrm{T}} F\left(\mathbf{s}_{\mathrm{E}}^{\prime} ; \mathbf{s}_{\mathrm{E}}^{\circ}\right)=\nabla_{\mathbf{s}^{\prime}}^{\mathrm{T}} F(\mathbf{s} ; \mathbf{s})+\mathbf{o}_{M}(\varepsilon), \\
& \nabla_{\mathbf{s}^{\circ}}^{\mathrm{T}} F\left(\mathbf{s}_{\mathrm{E}}^{\prime} ; \mathbf{s}_{\mathrm{E}}^{\circ}\right)=\nabla_{\mathbf{s}^{\circ}}^{\mathrm{T}} F(\mathbf{s} ; \mathbf{s})+\mathbf{o}_{M}(\varepsilon)=-\nabla_{\mathbf{s}^{\prime}}^{\mathrm{T}} F(\mathbf{s} ; \mathbf{s})+\mathbf{o}_{M}(\varepsilon),
\end{aligned}
$$

we find

with

$$
\begin{aligned}
F\left(\mathbf{s}_{\mathrm{h}}^{\prime} ; \mathbf{s}_{\mathrm{h}}^{\circ}\right) & =F\left(\mathbf{s}_{\mathrm{E}}^{\prime} ; \mathbf{s}_{\mathrm{E}}^{\circ}\right)-\nabla_{\mathbf{s}^{\prime}}^{\mathrm{T}} F(\mathbf{s} ; \mathbf{s}) \mathbf{B}^{+\mathrm{T}}\left[\mathbf{h}\left(\mathbf{s}_{\mathrm{E}}^{\prime}\right)-\mathbf{h}\left(\mathbf{s}_{\mathrm{E}}^{\circ}\right)\right]+O\left(\varepsilon^{3}\right) \\
& =F\left(\mathbf{s}_{\mathrm{E}}^{\prime} ; \mathbf{s}_{\mathrm{E}}^{\circ}\right)-\lambda_{\mathbf{s}}^{\mathrm{T}}\left[\mathbf{h}\left(\mathbf{s}_{\mathrm{E}}^{\prime}\right)-\mathbf{h}\left(\mathbf{s}_{\mathrm{E}}^{\circ}\right)\right]+O\left(\varepsilon^{3}\right)
\end{aligned}
$$

This completes the proof.

$$
\lambda_{\mathbf{s}}=\mathbf{B}^{+} \nabla_{\mathbf{s}^{\prime}} F(\mathbf{s} ; \mathbf{s})
$$

\section{Appendix E: Bordered second-derivative matrix}

\section{E.1. Parametric expression of constraint surface}

We consider a local parameterization of $\mathbf{h}(\mathbf{s})=\left(h_{L+1}(\mathbf{s}), \cdots, h_{M}(\mathbf{s})\right)^{\mathrm{T}}=\mathbf{0}$, by an $L$-dimensional vector $\boldsymbol{\phi}=\left(\phi_{1}, \cdots, \phi_{L}\right)^{\mathrm{T}}$. Without loss of generality we permute components of $\mathbf{s}=\left(x_{1}, \cdots, x_{M}\right)^{\mathrm{T}}$, so that $\mathbf{s}$ is separated into $\mathbf{x}=\left(x_{1}, \cdots, x_{L}\right)^{\mathrm{T}}$ and $\mathbf{y}=\left(x_{L+1}, \cdots, x_{M}\right)^{\mathrm{T}}$ that satisfies $\left|\nabla_{\mathbf{y}}^{\mathrm{T}} \mathbf{h}(\mathbf{s})\right| \neq 0$. Then at least locally we can choose $\boldsymbol{\phi}=\mathbf{x}$ so that a point on the constraint surface is expressed as $\mathbf{s}(\boldsymbol{\phi})=$ $(\mathbf{x}(\boldsymbol{\phi}), \mathbf{y}(\boldsymbol{\phi}))^{\mathrm{T}}=(\boldsymbol{\phi}, \mathbf{y}(\boldsymbol{\phi}))^{\mathrm{T}}$, in which case $F\left(\mathbf{s}^{\prime} ; \mathbf{s}^{\circ}\right)$ along the constraint surface is expressed as $\tilde{F}\left(\boldsymbol{\phi}^{\prime} ; \boldsymbol{\phi}^{\circ}\right)=F\left(\mathbf{s}\left(\boldsymbol{\phi}^{\prime}\right) ; \mathbf{s}\left(\boldsymbol{\phi}^{\circ}\right)\right)$. We expand $\mathbf{h}\left(\mathbf{s}^{\prime}\right)=\mathbf{0}$ in terms of $\boldsymbol{\phi}^{\prime}$ at $\mathbf{s}$ as

$$
\mathbf{h}\left(\mathbf{s}^{\prime}\right)=\nabla_{\boldsymbol{\phi}}^{\mathrm{T}} \mathbf{h}\left[\boldsymbol{\phi}^{\prime}-\boldsymbol{\phi}\right]+\frac{1}{2}\left[\boldsymbol{\phi}^{\prime}-\boldsymbol{\phi}\right]^{\mathrm{T}} \nabla_{\boldsymbol{\phi}} \nabla_{\boldsymbol{\phi}}^{\mathrm{T}} \mathbf{h}\left[\boldsymbol{\phi}^{\prime}-\boldsymbol{\phi}\right]+\mathbf{0}_{M-L}\left(\left|\boldsymbol{\phi}^{\prime}-\boldsymbol{\phi}\right|^{3}\right)=\mathbf{0},
$$

where 


$$
\begin{gathered}
\nabla_{\boldsymbol{\phi}}^{\mathrm{T}} \mathbf{h}=\left(\begin{array}{c}
\nabla_{\boldsymbol{\phi}}^{\mathrm{T}} h_{L+1} \\
\vdots \\
\nabla_{\boldsymbol{\phi}}^{\mathrm{T}} h_{M}
\end{array}\right)=\left(\begin{array}{ccc}
\frac{\partial h_{L+1}(\mathbf{s}(\boldsymbol{\phi}))}{\partial \phi_{1}} & \cdots & \frac{\partial h_{L+1}(\mathbf{s}(\boldsymbol{\phi}))}{\partial \phi_{L}} \\
\vdots & \ddots & \vdots \\
\frac{\partial h_{M}(\mathbf{s}(\boldsymbol{\phi}))}{\partial \phi_{1}} & \cdots & \frac{\partial h_{M}(\mathbf{s}(\boldsymbol{\phi}))}{\partial \phi_{L}}
\end{array}\right) \\
\nabla_{\boldsymbol{\phi}} \nabla_{\boldsymbol{\phi}}^{\mathrm{T}} \mathbf{h}=\left\{\begin{array}{c}
\nabla_{\boldsymbol{\phi}} \nabla_{\boldsymbol{\phi}}^{\mathrm{T}} h_{L+1} \\
\vdots \\
\nabla_{\boldsymbol{\phi}} \nabla_{\boldsymbol{\phi}}^{\mathrm{T}} h_{M}
\end{array}\right\}, \\
\nabla_{\boldsymbol{\phi}} \nabla_{\boldsymbol{\phi}}^{\mathrm{T}} h_{j}= \\
\left.\begin{array}{ccc}
\frac{\partial h_{j}(\mathbf{s}(\boldsymbol{\phi}))}{\partial \phi_{1}^{2}} & \cdots & \frac{\partial h_{j}(\mathbf{s}(\boldsymbol{\phi}))}{\partial \phi_{1} \partial \phi_{L}} \\
\vdots & \ddots & \vdots \\
\frac{\partial h_{j}(\mathbf{s}(\boldsymbol{\phi}))}{\partial \phi_{1} \partial \phi_{L}} & \cdots & \frac{\partial h_{j}(\mathbf{s}(\boldsymbol{\phi}))}{\partial \phi_{L}^{2}}
\end{array}\right)
\end{gathered}
$$

and all derivatives are evaluated at s, corresponding to $\boldsymbol{\phi}$ (see Eqs. (D.3-4) in Appendix D for operations for $\nabla_{\boldsymbol{\phi}} \nabla_{\boldsymbol{\phi}}^{\mathrm{T}} \mathbf{h}$ ). The first and second order terms in Eq.

(E.1) are transformed as

$$
\begin{aligned}
\nabla_{\boldsymbol{\phi}}^{\mathrm{T}} \mathbf{h}\left[\boldsymbol{\phi}^{\prime}-\boldsymbol{\phi}\right] & =\nabla_{\mathbf{s}}^{\mathrm{T}} \mathbf{h} \nabla_{\boldsymbol{\phi}}^{\mathrm{T}} \mathbf{s}\left[\boldsymbol{\phi}^{\prime}-\boldsymbol{\phi}\right] \\
\frac{1}{2}\left[\boldsymbol{\phi}^{\prime}-\boldsymbol{\phi}\right]^{\mathrm{T}} \nabla_{\boldsymbol{\phi}} \nabla_{\boldsymbol{\phi}}^{\mathrm{T}} \mathbf{h}\left[\boldsymbol{\phi}^{\prime}-\boldsymbol{\phi}\right] & =\frac{1}{2}\left[\boldsymbol{\phi}^{\prime}-\boldsymbol{\phi}\right]^{\mathrm{T}} \nabla_{\boldsymbol{\phi}}\left[\nabla_{\mathbf{s}}^{\mathrm{T}} \mathbf{h} \nabla_{\boldsymbol{\phi}}^{\mathrm{T}} \mathbf{s}\right]\left[\boldsymbol{\phi}^{\prime}-\boldsymbol{\phi}\right] \\
& =\frac{1}{2}\left[\boldsymbol{\phi}^{\prime}-\boldsymbol{\phi}\right]^{\mathrm{T}}\left[\left[\nabla_{\boldsymbol{\phi}}^{\mathrm{T}} \mathbf{s}\right]^{\mathrm{T}} \nabla_{\mathbf{s}} \nabla_{\mathbf{s}}^{\mathrm{T}} \mathbf{h} \nabla_{\boldsymbol{\phi}}^{\mathrm{T}} \mathbf{s}+\nabla_{\mathbf{s}}^{\mathrm{T}} \mathbf{h} \nabla_{\boldsymbol{\phi}} \nabla_{\boldsymbol{\phi}}^{\mathrm{T}} \mathbf{s}\right]\left[\boldsymbol{\phi}^{\prime}-\boldsymbol{\phi}\right],
\end{aligned}
$$

where

$$
\begin{gathered}
\nabla_{\boldsymbol{\phi}}^{\mathrm{T}} \mathbf{s}=\left(\begin{array}{c}
\nabla_{\boldsymbol{\phi}}^{\mathrm{T}} x_{1} \\
\vdots \\
\nabla_{\boldsymbol{\phi}}^{\mathrm{T}} x_{M}
\end{array}\right)=\left(\begin{array}{ccc}
\frac{\partial x_{1}}{\partial \phi_{1}} & \cdots & \frac{\partial x_{1}}{\partial \phi_{L}} \\
\vdots & \ddots & \vdots \\
\frac{\partial x_{M}}{\partial \phi_{1}} & \cdots & \frac{\partial x_{M}}{\partial \phi_{L}}
\end{array}\right), \\
\nabla_{\boldsymbol{\phi}} \nabla_{\boldsymbol{\phi}}^{\mathrm{T}} \mathbf{s}=\left\{\begin{array}{c}
\nabla_{\boldsymbol{\phi}} \nabla_{\boldsymbol{\phi}}^{\mathrm{T}} x_{1} \\
\vdots \\
\nabla_{\boldsymbol{\phi}} \nabla_{\boldsymbol{\phi}}^{\mathrm{T}} x_{M}
\end{array}\right\}, \quad \nabla_{\boldsymbol{\phi}} \nabla_{\boldsymbol{\phi}}^{\mathrm{T}} x_{i}=\left(\begin{array}{ccc}
\frac{\partial x_{i}}{\partial \phi_{1}^{2}} & \cdots & \frac{\partial x_{i}}{\partial \phi_{1} \partial \phi_{L}} \\
\vdots & \ddots & \vdots \\
\frac{\partial h_{i}}{\partial \phi_{1} \partial \phi_{L}} & \cdots & \frac{\partial x_{i}}{\partial \phi_{L}^{2}}
\end{array}\right),
\end{gathered}
$$

and 


$$
\begin{gathered}
\nabla_{\mathbf{s}}^{\mathrm{T}} \mathbf{h}=\left(\begin{array}{c}
\nabla_{\mathbf{s}}^{\mathrm{T}} h_{L+1} \\
\vdots \\
\nabla_{\mathbf{s}}^{\mathrm{T}} h_{M}
\end{array}\right)=\left(\begin{array}{ccc}
\frac{\partial h_{L+1}(\mathbf{s})}{\partial x_{1}} & \cdots & \frac{\partial h_{L+1}(\mathbf{s})}{\partial x_{M}} \\
\vdots & \ddots & \vdots \\
\frac{\partial h_{M}(\mathbf{s})}{\partial x_{1}} & \cdots & \frac{\partial h_{M}(\mathbf{s})}{\partial x_{M}}
\end{array}\right), \\
\nabla_{\mathbf{s}} \nabla_{\mathbf{s}}^{\mathrm{T}} \mathbf{h}=\left\{\begin{array}{c}
\nabla_{\mathbf{s}} \nabla_{\mathbf{s}}^{\mathrm{T}} h_{L+1} \\
\vdots \\
\nabla_{\mathbf{s}} \nabla_{\mathbf{s}}^{\mathrm{T}} h_{M}
\end{array}\right\}, \quad \nabla_{\mathbf{s}} \nabla_{\mathbf{s}}^{\mathrm{T}} h_{j}=\left(\begin{array}{ccc}
\frac{\partial h_{j}(\mathbf{s})}{\partial x_{1}^{2}} & \cdots & \frac{\partial h_{j}(\mathbf{s})}{\partial x_{1} \partial x_{M}} \\
\vdots & \ddots & \vdots \\
\frac{\partial h_{j}(\mathbf{s})}{\partial x_{1} \partial x_{M}} & \cdots & \frac{\partial h_{j}(\mathbf{s})}{\partial x_{M}^{2}}
\end{array}\right),
\end{gathered}
$$

where $\nabla_{\mathbf{s}}^{\mathrm{T}} h_{j}$ and $\nabla_{\mathbf{s}} \nabla_{\mathbf{s}}^{\mathrm{T}} h_{j}$ are identical to $\nabla^{\mathrm{T}} h_{j}$ and $\nabla \nabla^{\mathrm{T}} h_{j}$ in the main text. Since Eq. (E.1) holds for any $\boldsymbol{\phi}^{\prime}-\boldsymbol{\phi}$, from Eqs. (E.3) we see

$$
\begin{gathered}
\nabla_{\mathbf{s}}^{\mathrm{T}} \mathbf{h} \nabla_{\boldsymbol{\phi}}^{\mathrm{T}} \mathbf{s}=\mathbf{B}^{\mathrm{T}} \mathbf{E R}=\mathbf{0}, \\
{\left[\nabla_{\boldsymbol{\phi}}^{\mathrm{T}} \mathbf{s}\right]^{\mathrm{T}} \nabla_{\mathbf{s}} \nabla_{\mathbf{s}}^{\mathrm{T}} \mathbf{h} \nabla_{\boldsymbol{\phi}}^{\mathrm{T}} \mathbf{s}+\nabla_{\mathbf{s}}^{\mathrm{T}} \mathbf{h} \nabla_{\boldsymbol{\phi}} \nabla_{\boldsymbol{\phi}}^{\mathrm{T}} \mathbf{s}=[\mathbf{E R}]^{\mathrm{T}} \nabla_{\mathbf{s}} \nabla_{\mathbf{s}}^{\mathrm{T}} \mathbf{h E R}+\mathbf{B}^{\mathrm{T}} \nabla_{\boldsymbol{\phi}} \nabla_{\boldsymbol{\phi}}^{\mathrm{T}} \mathbf{s}=\mathbf{0},}
\end{gathered}
$$

where $\mathbf{B}^{\mathrm{T}}=\nabla_{\mathbf{s}}^{\mathrm{T}} \mathbf{h}$, ER $=\nabla_{\phi}^{\mathrm{T}} \mathbf{S}$, where $\mathbf{R}$ is an appropriately chosen regular matrix whose inverse normalizes the base vectors of the tangent plane of the surface, $\nabla_{\boldsymbol{\phi}}^{\mathrm{T}} \mathbf{s}=\left(\tilde{\mathbf{e}}_{1}, \cdots, \tilde{\mathbf{e}}_{L}\right)$, into orthogonal unit base vectors $\mathbf{E}=\left(\mathbf{e}_{1}, \cdots, \mathbf{e}_{L}\right)$ (e.g., $\mathbf{R}^{-1}$ is Gram-Schmidt orthonormalization with scaling of each base vector). From Eq. (E.7), we see

$$
\nabla_{\boldsymbol{\phi}} \nabla_{\boldsymbol{\phi}}^{\mathrm{T}} \mathbf{s}=-\mathbf{B}^{+\mathrm{T}}[\mathbf{E R}]^{\mathrm{T}} \nabla_{\mathbf{s}} \nabla_{\mathbf{s}}^{\mathrm{T}} \mathbf{h E R},
$$

where $\mathbf{B}^{+}=\left[\mathbf{B}^{\mathrm{T}} \mathbf{B}\right]^{-1} \mathbf{B}$. Thus, the first and second derivatives of unconstrained $L$ dimensional invasion fitness $\tilde{F}\left(\boldsymbol{\phi}^{\prime} ; \boldsymbol{\phi}^{\circ} ; \boldsymbol{\lambda}_{\boldsymbol{\phi}}\right)$ with respect to $\boldsymbol{\phi}^{\prime}$ at $\boldsymbol{\phi}$ are expressed as

$$
\nabla_{\boldsymbol{\phi}^{\prime}}^{\mathrm{T}} \tilde{F}=\nabla_{\mathbf{s}^{\prime}}^{\mathrm{T}} F \nabla_{\boldsymbol{\phi}}^{\mathrm{T}} \mathbf{s}=\mathbf{g}^{\mathrm{T}} \mathbf{E R},
$$

and

$$
\begin{aligned}
\mathbf{D}_{\phi}=\nabla_{\boldsymbol{\phi}^{\prime}} \nabla_{\boldsymbol{\phi}^{\prime}}^{\mathrm{T}} \tilde{F} & =\left[\nabla_{\boldsymbol{\phi}}^{\mathrm{T}} \mathbf{s}\right]^{\mathrm{T}} \nabla_{\mathbf{s}^{\prime}} \nabla_{\mathbf{s}^{\prime}}^{\mathrm{T}} F \nabla_{\boldsymbol{\phi}}^{\mathrm{T}} \mathbf{s}+\nabla_{\mathbf{s}^{\prime}}^{\mathrm{T}} F \nabla_{\boldsymbol{\phi}} \nabla_{\boldsymbol{\phi}}^{\mathrm{T}} \mathbf{s}, \\
& =[\mathbf{E R}]^{\mathrm{T}} \nabla_{\mathbf{s}^{\prime}} \nabla_{\mathbf{s}^{\prime}}^{\mathrm{T}} F \mathbf{E R}-\mathbf{g}^{\mathrm{T}} \mathbf{B}^{+}[\mathbf{E R}]^{\mathrm{T}} \nabla_{\mathbf{s}} \nabla_{\mathbf{s}}^{\mathrm{T}} \mathbf{h E R} \\
& =[\mathbf{E R}]^{\mathrm{T}} \nabla_{\mathbf{s}^{\prime}} \nabla_{\mathbf{s}^{\prime}}^{\mathrm{T}} F \mathbf{E R}-\lambda_{\mathbf{s}}^{\mathrm{T}}[\mathbf{E R}]^{\mathrm{T}} \nabla_{\mathbf{s}} \nabla_{\mathbf{s}}^{\mathrm{T}} \mathbf{h} \mathbf{E R} \\
& =[\mathbf{E R}]^{\mathrm{T}}\left[\nabla_{\mathbf{s}^{\prime}} \nabla_{\mathbf{s}^{\prime}}^{\mathrm{T}} F-\sum_{j=L+1}^{M} \lambda_{\mathbf{s}, j} \nabla_{\mathbf{s}} \nabla_{\mathbf{s}}^{\mathrm{T}} h_{j}\right] \mathbf{E R} \\
& =[\mathbf{E R}]^{\mathrm{T}}\left[\nabla_{\mathbf{s}^{\prime}} \nabla_{\mathbf{s}^{\prime}}^{\mathrm{T}} F_{L}\right] \mathbf{E R}=[\mathbf{E R}]^{\mathrm{T}} \mathbf{D}_{\mathrm{L}} \mathbf{E R},
\end{aligned}
$$


where $\nabla_{\mathbf{s}^{\prime}} \nabla_{\mathbf{s}^{\prime}}^{\mathrm{T}} F_{L}$ is the second derivative of the Lagrange fitness function defined by Eq. (15) with Lagrange multiplier for $\boldsymbol{\lambda}_{\mathbf{s}}=\mathbf{B}^{+} \mathbf{g}$ given by Eq. (19).

\section{E.2. Stability conditions along constraint surface}

Symmetric matrix $\mathbf{D}_{\phi}$ is positive definite if its all principal minors given by

$$
\left|\mathbf{D}_{\phi}^{(i)}\right|=\left|\left(\begin{array}{ccc}
D_{\phi, 11} & \cdots & D_{\phi, 1 i} \\
\vdots & \ddots & \vdots \\
D_{\phi, i 1} & \cdots & D_{\phi, i i}
\end{array}\right)\right|
$$

$i=1, \cdots, L$ are positive. According to Mandy (2013), $\left|\mathbf{D}_{\phi}^{(i)}\right|$ satisfies the following relationship

$$
\left.\left|\left(\begin{array}{cc}
\mathbf{0} & \mathbf{B}^{\mathrm{T}} \\
\mathbf{B} & \mathbf{D}_{\mathrm{L}}^{(\tilde{\imath})}
\end{array}\right)^{(\tilde{\imath})}=(-1)^{N}\right| \mathbf{B}_{\mathbf{y}}\right|^{2}\left|\mathbf{D}_{\phi}^{(i)}\right|
$$

with $\tilde{\imath}=i+2 N$ (i.e., $\tilde{\imath}=2 N+1, \cdots, N+M$ ), where $N=M-L$ is the number of equality constraints, and $\mathbf{B}^{\mathrm{T}}=\left(\mathbf{B}_{\mathbf{x}}^{\mathrm{T}}, \mathbf{B}_{\mathbf{y}}^{\mathrm{T}}\right)=\left(\nabla_{\mathbf{x}}^{\mathrm{T}} \mathbf{h}, \nabla_{\mathbf{y}}^{\mathrm{T}} \mathbf{h}\right)$. Substituting Eq. (E.10) into Eq. (E.12) gives

$$
\left|\left(\begin{array}{ll}
\mathbf{0} & \mathbf{B}^{\mathrm{T}} \\
\mathbf{B} & \mathbf{D}_{\mathrm{L}}
\end{array}\right)^{(\tilde{\imath})}\right|=(-1)^{N}\left|\mathbf{B}_{\mathbf{y}}\right|^{2}\left|\left[\mathbf{R}^{\mathrm{T}} \mathbf{E}^{\mathrm{T}} \mathbf{D}_{\mathrm{L}} \mathbf{E R}\right]^{(i)}\right|
$$

Thus, if the left side of Eq. (E.13) has the sign $(-1)^{N}$ for all $\tilde{\imath}=2 N+1, \cdots, N+M$, then $\mathbf{R}^{\mathrm{T}} \mathbf{E}^{\mathrm{T}} \mathbf{D}_{\mathrm{L}} \mathbf{E R}$ is positive definite, in which case $\mathbf{E}^{\mathrm{T}} \mathbf{D}_{\mathrm{L}} \mathbf{E}$ is also positive definite because $\mathbf{v}^{\mathrm{T}} \mathbf{R}^{\mathrm{T}} \mathbf{E}^{\mathrm{T}} \mathbf{D}_{\mathrm{L}} \mathbf{E R v}>0$ for any $\mathbf{v}$ gives $\tilde{\mathbf{v}}^{\mathrm{T}} \mathbf{E}^{\mathrm{T}} \mathbf{D}_{\mathrm{L}} \mathbf{E} \tilde{\mathbf{v}}>0$ for any $\tilde{\mathbf{v}}=\mathbf{R v}$.

Conversely, if the left side of Eq. (E.13) has different sign from $(-1)^{N}$ for either of $\tilde{\imath}=2 N+1, \cdots, N+M$, then $\mathbf{E}^{\mathrm{T}} \mathbf{D}_{\mathrm{L}} \mathbf{E}$ has at least one negative eigenvalue.

Since Eq. (E.13) clearly holds good for an arbitrary $M \times M$ symmetric matrix, we can replace $\mathbf{D}_{\mathrm{L}}$ in Eq. (E. 13) with $-\mathbf{D}_{\mathrm{L}}$, and define

$$
\mathbf{D}_{\mathrm{B}}=\left(\begin{array}{cc}
\mathbf{0} & \mathbf{B}^{\mathrm{T}} \\
\mathbf{B} & -\mathbf{D}_{\mathrm{L}}
\end{array}\right) \text {. }
$$

Clearly, if $\left|\mathbf{D}_{\mathrm{B}}^{(\tilde{\imath})}\right|$ has the sign $(-1)^{N}$ for all $\tilde{\imath}=2 N+1, \cdots, N+M$, then $\mathbf{E}^{\mathrm{T}} \mathbf{D}_{\mathrm{L}} \mathbf{E}$ is negative definite (i.e., evolutionarily stable). Conversely, if $\left|\mathbf{D}_{\mathrm{B}}^{(\tilde{J})}\right|$ has the opposite sign from $(-1)^{N}$ for either of $\tilde{\imath}=2 N+1, \cdots, N+M$, then $\mathbf{E}^{\mathrm{T}} \mathbf{D}_{\mathrm{L}} \mathbf{E}$ has at least one positive eigenvalue (i.e., evolutionarily unstable). These statements are Corollary 1 in the main text. Similarly, by replacing $\mathbf{D}_{\mathrm{L}}$ with $-1 / 2\left[\mathbf{C}_{\mathrm{L}}+\mathbf{C}_{\mathrm{L}}^{\mathrm{T}}\right]$ and $-1 / 2[\mathbf{M}+\mathbf{M}]$ in Eq. (E. 13) give Corollaries 2 and 3, respectively, in the main text.

For a one-dimensional constraint curve in a two-dimensional trait space $\mathbf{s}=$ $(x, y)^{\mathrm{T}}$, i.e., $N=1$ and $M=2$, the $\left|\mathbf{B}_{\mathbf{y}}\right|^{2}, \mathbf{E}$, and $\mathbf{R}$ in Eq. (E.13) are, respectively, a scalar $[\partial h / \partial y]^{2}$, a unit vector $\mathbf{e}=\left(n_{\mathrm{y}},-n_{\mathrm{x}}\right)^{\mathrm{T}}$ with $\left(n_{\mathrm{x}}, n_{\mathrm{y}}\right)^{\mathrm{T}}=\nabla_{\mathbf{s}} h /\left|\nabla_{\mathbf{s}} h\right|$, and a 
scalar $R=\left|\nabla_{\mathbf{s}} h\right|\left[\frac{\partial h}{\partial y}\right]^{-1}$ (specified from $R \mathbf{e}=\frac{\mathrm{d} \mathbf{s}}{\mathrm{d} \phi}=\left(\frac{\mathrm{d} x}{\mathrm{~d} \phi}, \frac{\mathrm{d} y}{\mathrm{~d} \phi}\right)^{\mathrm{T}}=\left(1, \frac{\mathrm{d} y}{\mathrm{~d} \phi}\right)^{\mathrm{T}}$ ). In this case, we can simplify Eq. (E.13), with replacement of $\mathbf{D}_{\mathrm{L}}$ with $-\mathbf{D}_{\mathrm{L}}$, into

Similarly, we find

$$
\mathbf{e}^{\mathrm{T}} \mathbf{D}_{\mathrm{L}} \mathbf{e}=\frac{\left|\mathbf{D}_{\mathrm{B}}\right|}{\left|\nabla_{\mathbf{s}} h\right|^{2}}
$$

$$
\begin{aligned}
\mathbf{e}^{\mathrm{T}} \mathbf{C}_{\mathrm{L}} \mathbf{e} & =\frac{\left|\mathbf{C}_{\mathrm{B}}\right|}{\left|\nabla_{\mathbf{s}} h\right|^{2}} \\
\mathbf{e}^{\mathrm{T}} \mathbf{M e} & =\frac{\left|\mathbf{M}_{\mathrm{B}}\right|}{\left|\nabla_{\mathbf{s}} h\right|^{2}}
\end{aligned}
$$

\section{Appendix F: Curvature index for multi- dimensional constraint surfaces}

As explained in the main text, in the case of two-dimensional trait spaces, the effect of the curvature of the constraint curve, $\Omega$, is the inner product of the fitness gradient $\mathbf{g}=\nabla_{\mathbf{s}^{\prime}} F(\mathbf{s} ; \mathbf{s})$ and the curvature vector $\mathbf{q}$ that specifies the local curvature and its orientation of the constraint curve. Similar relationship is derived in the higher-dimensional constraint surfaces, by extending the definitions of inner product and curvature vector. Specifically, as explained below, the effect of the curvature is expressed as

$$
\boldsymbol{\Omega}=\left\langle\mathbf{g}^{\mathrm{T}}, \mathrm{Q}\right\rangle,
$$

where the apparent fitness curvature $\boldsymbol{\Omega}$ is extended to an $L$-by- $L$ matrix given by an extended inner product $\langle$,$\rangle of the fitness gradient \mathbf{g}$ and a constraint curvature $\mathrm{Q}$, which is a vector having matrices as its components. Below, we derive this equation from the definition of $\boldsymbol{\Omega}$ in the main text, Eq. (23b).

First, we denote $(j, k)$ th component of the matrix $\mathbf{B}^{+}$in Eq. (19) by $b_{j k}^{+}$for $j=L+1, \cdots, M$ and $k=1, \cdots, M$. Then $\lambda_{\mathbf{s}}$ is transformed as 


$$
\begin{aligned}
\boldsymbol{\lambda}_{\mathbf{s}} & =\mathbf{B}^{+} \mathbf{g} \\
& =\left(\begin{array}{ccc}
b_{L+1,1}^{+} & \cdots & b_{L+1, M}^{+} \\
\vdots & \ddots & \vdots \\
b_{M, 1}^{+} & \cdots & b_{M, M}^{+}
\end{array}\right)\left(\begin{array}{c}
g_{1} \\
\vdots \\
g_{M}
\end{array}\right) \\
& =\left(\begin{array}{c}
\sum_{k=1}^{M} b_{L+1, k}^{+} g_{k} \\
\vdots \\
\sum_{k=1}^{M} b_{M, k}^{+} g_{k}
\end{array}\right),
\end{aligned}
$$

where $\mathbf{g}=\left(g_{1}, \cdots, g_{M}\right)^{\mathrm{T}}$. Substituting this equation into Eq. (23b) gives

$$
\begin{aligned}
\mathbf{\Omega} & =-\sum_{j=L+1}^{M} \lambda_{\mathbf{s} j} \mathbf{E}^{\mathrm{T}} \nabla \nabla^{\mathrm{T}} h_{j}(\mathbf{s}) \mathbf{E} . \\
& =-\sum_{j=L+1}^{M} \sum_{k=1}^{M} b_{j, k}^{+} g_{k} \mathbf{E}^{\mathrm{T}} \nabla \nabla^{\mathrm{T}} h_{j}(\mathbf{s}) \mathbf{E} \\
& =-\sum_{k=1}^{M} g_{k} \sum_{j=L+1}^{M} b_{j, k}^{+} \mathbf{E}^{\mathrm{T}} \nabla \nabla^{\mathrm{T}} h_{j}(\mathbf{s}) \mathbf{E} \\
& =\sum_{k=1}^{M} g_{k} \mathbf{Q}_{k},
\end{aligned}
$$

where $\mathbf{Q}_{k}$ is an $L$-by- $L$ matrix given by

$$
\mathbf{Q}_{k}=-\sum_{j=L+1}^{M} b_{j, k}^{+} \mathbf{E}^{\mathrm{T}} \nabla \nabla^{\mathrm{T}} h_{j}(\mathbf{s}) \mathbf{E} .
$$

Finally, Eq. (F.3) is transformed into Eq. (F.1) $\boldsymbol{\Omega}=\left\langle\mathbf{g}^{\mathrm{T}}, \mathrm{Q}\right\rangle$ by defining an inner product $\langle$,$\rangle ,$

$$
\left\langle\mathbf{g}^{\mathrm{T}}, \mathrm{Q}\right\rangle=\left\langle\left(g_{1}, \cdots, g_{M}\right),\left\{\begin{array}{c}
\mathbf{Q}_{1} \\
\vdots \\
\mathbf{Q}_{M}
\end{array}\right\}\right)=\sum_{k=1}^{M} g_{k} \mathbf{Q}_{k},
$$

where $\mathrm{Q}$ is a vector having $L$-by- $L$ matrices as its components,

$$
\mathrm{Q}=\left\{\begin{array}{c}
\mathbf{Q}_{1} \\
\vdots \\
\mathbf{Q}_{M}
\end{array}\right\}
$$

Moreover, by the extended inner product, Eq. (F.4) is further transformed into 


$$
\mathbf{Q}_{k}=-\left\langle\mathbf{b}_{\mathrm{k}}^{+\mathrm{T}}, \mathrm{H}_{\mathrm{E}}\right\rangle
$$

with $\mathbf{b}_{\mathrm{k}}^{+\mathrm{T}}=\left(b_{L+1, k}^{+}, \cdots, b_{M, k}^{+}\right)$and

$$
\begin{aligned}
H_{\mathrm{E}}=\left\{\begin{array}{c}
\mathbf{H}_{\mathrm{E}, L+1} \\
\vdots \\
\mathbf{H}_{\mathrm{E}, M}
\end{array}\right\} & =\left\{\begin{array}{c}
\mathbf{E}^{\mathrm{T}} \nabla \nabla^{\mathrm{T}} h_{L+1}(\mathbf{s}) \mathbf{E} \\
\vdots \\
\mathbf{E}^{\mathrm{T}} \nabla \nabla^{\mathrm{T}} h_{M}(\mathbf{s}) \mathbf{E}
\end{array}\right\}=\mathbf{E}^{\mathrm{T}} \mathrm{HE}, \\
\mathbf{H} & =\left\{\begin{array}{c}
\nabla \nabla^{\mathrm{T}} h_{L+1}(\mathbf{s}) \\
\vdots \\
\nabla \nabla^{\mathrm{T}} h_{M}(\mathbf{s})
\end{array}\right\}
\end{aligned}
$$

(see Eq. (D.4) in Appendix D for the last transformation in Eq. (E.8)). Then by defining

$$
\left\langle\mathbf{B}^{+\mathrm{T}}, \mathrm{H}_{\mathrm{E}}\right\rangle=\left\langle\left(\begin{array}{c}
\mathbf{b}_{1}^{+\mathrm{T}} \\
\vdots \\
\mathbf{b}_{M}^{+\mathrm{T}}
\end{array}\right), \mathrm{H}_{\mathrm{E}}\right\rangle=\left\{\begin{array}{c}
\left\langle\mathbf{b}_{1}^{+\mathrm{T}}, \mathrm{H}_{\mathrm{E}}\right\rangle \\
\vdots \\
\left\langle\mathbf{b}_{M}^{+\mathrm{T}}, \mathrm{H}_{\mathrm{E}}\right\rangle
\end{array}\right\},
$$

we obtain

$$
Q=\left\{\begin{array}{c}
\left\langle\mathbf{b}_{1}^{+\mathrm{T}}, \mathrm{H}_{\mathrm{E}}\right\rangle \\
\vdots \\
\left\langle\mathbf{b}_{M}^{+\mathrm{T}}, \mathrm{H}_{\mathrm{E}}\right\rangle
\end{array}\right\}=-\left\langle\mathbf{B}^{+\mathrm{T}}, \mathrm{H}_{\mathrm{E}}\right\rangle=-\left\langle\mathbf{B}^{+\mathrm{T}}, \mathbf{E}^{\mathrm{T}} \mathrm{HE}\right\rangle .
$$

This $Q$ has information about the local curvature of the constraint surface, and its form is similar to that of the constraint curvature vector $\mathbf{q}$ for two-dimensional trait spaces, because Eqs. (13) can be transformed into

$$
\begin{aligned}
\mathbf{q} & =-\mathbf{b}^{+\mathrm{T}}\left[\mathbf{e}^{\mathrm{T}} \nabla \nabla^{\mathrm{T}} h(\mathbf{s}) \mathbf{e}\right], \\
\mathbf{b}^{+} & =\frac{\nabla h^{\mathrm{T}}}{|\nabla h|^{2}} .
\end{aligned}
$$

Notice that $\mathbf{E}=\left(\mathbf{e}_{1}, \cdots, \mathbf{e}_{L}\right)$ and $\mathrm{H}$ given by Eq. (F.9) are multi-dimensional extensions of $\mathbf{e}^{\mathrm{T}}$ and $\nabla \nabla^{\mathrm{T}} h(\mathbf{s})$, respectively. Also $\mathbf{B}^{+}=\left[\mathbf{B}^{\mathrm{T}} \mathbf{B}\right]^{-1} \mathbf{B}^{\mathrm{T}}$, the pseud inverse of $\mathbf{B}=\left(\nabla h_{L+1}(\mathbf{s}), \cdots, \nabla h_{M}(\mathbf{s})\right)$, is a multi-dimensional extension of of $\mathbf{b}^{+}$ that is the pseud inverse of $\nabla h(\mathbf{s})$, i.e., $\nabla h(\mathbf{s})^{+}=\left[\nabla h(\mathbf{s})^{\mathrm{T}} \nabla h(\mathbf{s})\right]^{-1} \nabla h(\mathbf{s})^{\mathrm{T}}=\mathbf{b}^{+}$.

\section{Appendix G: Proof of Theorem 3}

The branching potential matrix Eq. (24a)

$$
\mathbf{P}=\frac{1}{2} \mathbf{U}^{\mathrm{T}}\left[\mathbf{M}+\mathbf{M}^{\mathrm{T}}\right] \mathbf{U}
$$

has a zero eigenvalue in the direction of $\mathbf{g}$, since $\mathbf{U g}=\left[\mathbf{I}-\left(\mathbf{g g}^{\mathrm{T}}\right) /\left.\mathbf{| g}\right|^{2}\right] \mathbf{g}=\mathbf{g}-$ $\mathbf{g}=\mathbf{0}$ gives $\mathbf{P g}=\mathbf{0}$. Thus, the symmetric matrix $\mathbf{P}$ can be diagonalized as 


$$
\mathbf{P}=\left(\mathbf{v}_{1}, \ldots, \mathbf{v}_{M-1}, \mathbf{v}_{M}\right)\left(\begin{array}{cccc}
\alpha_{1} & \cdots & \cdots & 0 \\
\vdots & \ddots & \ddots & \vdots \\
\vdots & \ddots & \alpha_{M-1} & \vdots \\
0 & \cdots & \cdots & 0
\end{array}\right)\left(\begin{array}{c}
\mathbf{v}_{1}^{\mathrm{T}} \\
\vdots \\
\mathbf{v}_{M-1}^{\mathrm{T}} \\
\mathbf{v}_{M}^{\mathrm{T}}
\end{array}\right)
$$

with the real eigenvalues $\alpha_{1}, \cdots, \alpha_{M-1}, 0$ and the corresponding orthogonal eigenvectors $\mathbf{v}_{1}, \cdots, \mathbf{v}_{M-1}, \mathbf{v}_{M}$, where

$$
\mathbf{v}_{M}=\frac{\mathbf{g}}{|\mathbf{g}|} .
$$

Below we prove that if either of $\alpha_{1}, \cdots, \alpha_{M-1}$ is positive then $\mathbf{s}$ is a CBP along a constraint surface $\mathbf{h}\left(\mathbf{s}^{\prime}\right)=\left(h_{L+1}\left(\mathbf{s}^{\prime}\right), \cdots, h_{M}\left(\mathbf{s}^{\prime}\right)\right)^{\mathrm{T}}=\mathbf{0}$ of an arbitrary $L$ dimension $(1 \leq L \leq M-1)$, with appropriate choice of its first and second derivatives. For convenience, we permute the column of $\left(\mathbf{v}_{1}, \ldots, \mathbf{v}_{M-1}, \mathbf{v}_{M}\right)$ in Eq. (G.1) so that $\alpha_{1}>\alpha_{2}>\cdots>\alpha_{M-1}$, and assume that the first $K$ eigenvalues are positive, i.e., $\alpha_{1}>\alpha_{2}>\cdots>\alpha_{K}>0$, where $1 \leq K \leq M-1$. We choose $\mathbf{h}\left(\mathbf{s}^{\prime}\right)$ to satisfy for $j=L+1, \ldots, M-1$,

$$
\nabla h_{j}(\mathbf{s})=\mathbf{v}_{j}
$$

and for $j=M$,

$$
\begin{aligned}
\nabla h_{M}(\mathbf{s}) & =\mathbf{g}=|\mathbf{g}| \mathbf{v}_{M}, \\
\nabla \nabla^{\mathrm{T}} h_{M}(\mathbf{s}) & =\frac{1}{2}\left(\mathbf{C}+\mathbf{C}^{T}\right)+\tilde{\varepsilon} \mathbf{I},
\end{aligned}
$$

with a positive and sufficiently small $\widetilde{\varepsilon}$. Note that Eqs. (G.4-5) are equivalent to Eq. (24b). The remaining eigenvectors $\mathbf{v}_{1}, \cdots, \mathbf{v}_{L}$ are used as the orthogonal base vectors $\mathbf{e}_{1}, \cdots, \mathbf{e}_{L}$ for the tangent plane of the surface at $\mathbf{s}$, combined into a matrix

$$
\mathbf{E}=\left(\mathbf{e}_{1}, \cdots, \mathbf{e}_{L}\right)=\left(\mathbf{v}_{1}, \cdots, \mathbf{v}_{L}\right) .
$$

When $L=M-1$, Eq. (G.3) is omitted. When $L=1$, the constraint surface is onedimensional (constraint curve), and $\mathbf{E}$ becomes a vector $\mathbf{e}_{1}$.

\section{G.1. Lagrange fitness function}

According to Eqs. (15) and (19), the Lagrange fitness function is constructed as

$$
F_{\mathrm{L}}\left(\mathbf{s}^{\prime} ; \mathbf{s}^{\circ} ; \boldsymbol{\lambda}_{\mathbf{s}}\right)=F\left(\mathbf{s}^{\prime} ; \mathbf{s}^{\circ}\right)-\lambda_{\mathbf{s}}^{\mathrm{T}}\left[\mathbf{h}\left(\mathbf{s}^{\prime}\right)-\mathbf{h}\left(\mathbf{s}^{\circ}\right)\right],
$$

with $\lambda_{\mathbf{s}}=\left(\lambda_{\mathbf{s}, L+1}, \cdots, \lambda_{\mathbf{s}, M}\right)^{\mathrm{T}}=\mathbf{B}^{+} \mathbf{g}$, where $\mathbf{B}^{+}=\left[\mathbf{B}^{\mathrm{T}} \mathbf{B}\right]^{-1} \mathbf{B}^{\mathrm{T}}$ and $\mathbf{B}=$ $\left(\nabla h_{L+1}(\mathbf{s}), \cdots, \nabla h_{M}(\mathbf{s})\right)=\left(\mathbf{v}_{L+1}, \cdots, \mathbf{v}_{M-1},|\mathbf{g}| \mathbf{v}_{M}\right)$. Because $\mathbf{v}_{L+1}, \cdots, \mathbf{v}_{M}$ are orthogonal, $\mathbf{B}^{+}$is given by 


$$
\begin{aligned}
& \mathbf{B}^{+}=\left[\mathbf{B}^{\mathrm{T}} \mathbf{B}\right]^{-1} \mathbf{B}^{\mathrm{T}} \\
& =\left[\left(\begin{array}{c}
\nabla h_{L+1}(\mathbf{s})^{\mathrm{T}} \\
\vdots \\
\nabla h_{M}(\mathbf{s})^{\mathrm{T}}
\end{array}\right)\left(\nabla h_{L+1}(\mathbf{s}), \cdots, \nabla h_{M}(\mathbf{s})\right)\right]^{-1} \mathbf{B}^{\mathrm{T}} \\
& =\left(\begin{array}{ccc}
\left|\nabla h_{L+1}(\mathbf{s})\right|^{2} & \cdots & 0 \\
\vdots & \ddots & \vdots \\
0 & \cdots & \left|\nabla h_{M}(\mathbf{s})\right|^{2}
\end{array}\right)^{-1}\left(\begin{array}{c}
\nabla h_{L+1}(\mathbf{s})^{\mathrm{T}} \\
\vdots \\
\nabla h_{M}(\mathbf{s})^{\mathrm{T}}
\end{array}\right) \\
& =\left(\begin{array}{c}
\frac{\nabla h_{L+1}(\mathbf{s})^{\mathrm{T}}}{\left|\nabla h_{L+1}(\mathbf{s})\right|^{2}} \\
\vdots \\
\frac{\nabla h_{M}(\mathbf{s})^{\mathrm{T}}}{\left|\nabla h_{M}(\mathbf{s})\right|^{2}}
\end{array}\right) \\
& =\left(\begin{array}{c}
\mathbf{v}_{L+1}^{\mathrm{T}} \\
\vdots \\
\mathbf{v}_{M-1}^{\mathrm{T}} \\
\mathbf{v}_{M}^{\mathrm{T}} /|\mathbf{g}|
\end{array}\right),
\end{aligned}
$$

by which $\boldsymbol{\lambda}_{\mathbf{s}}$ is transformed as

$$
\begin{gathered}
\lambda_{\mathbf{s}}=\mathbf{B}^{+} \mathbf{g}=\left(\begin{array}{c}
\mathbf{v}_{L+1}^{\mathrm{T}} \\
\vdots \\
\mathbf{v}_{M-1}^{\mathrm{T}} \\
\mathbf{v}_{M}^{\mathrm{T}} \\
|\mathbf{g}|
\end{array}\right) \mathbf{g} \\
=\left(\begin{array}{c}
\mathbf{v}_{L+1}^{\mathrm{T}} \mathbf{g} \\
\vdots \\
\mathbf{v}_{M-1}^{\mathrm{T}} \mathbf{g} \\
\frac{\mathbf{v}_{M}^{\mathrm{T}} \mathbf{g}}{|\mathbf{g}|}
\end{array}\right) \\
=\left(\begin{array}{c}
0 \\
\vdots \\
0 \\
1
\end{array}\right) .
\end{gathered}
$$




\section{G.2. Checking conditions for evolutionary branching}

Now we apply Theorem 2 to the Lagrange fitness function Eq. (G.7) with $\lambda_{\mathbf{s}}=$ $(0, \cdots, 0,1)^{\mathrm{T}}$. First, $\mathbf{s}$ satisfies condition (i) for evolutionary singularity along the constraint surface,

$$
\begin{aligned}
\nabla F_{\mathrm{L}}\left(\mathbf{s} ; \mathbf{s} ; \boldsymbol{\lambda}_{\mathbf{s}}\right) & =\mathbf{g}-\lambda_{\mathbf{s}}^{\mathrm{T}} \nabla \mathbf{h}(\mathbf{s}) \\
& =\mathbf{g}-\nabla h_{M}(\mathbf{s}) \\
& =\mathbf{0} .
\end{aligned}
$$

Second, in order to examine condition (ii) for the strong convergence stability of $\mathbf{s}$, the effect of the curvature of the constraint surface is calculated by Eq. (23b) in the main text and Eq. (G.5) as

$$
\begin{aligned}
\boldsymbol{\Omega} & =-\sum_{j=L+1}^{M} \lambda_{\mathbf{s}, j} \mathbf{E}^{\mathrm{T}} \nabla \nabla^{\mathrm{T}} h_{j}(\mathbf{s}) \mathbf{E} \\
& =-\mathbf{E}^{\mathrm{T}} \nabla \nabla^{\mathrm{T}} h_{M}(\mathbf{s}) \mathbf{E} \\
& =-\mathbf{E}^{\mathrm{T}}\left[\frac{1}{2}\left(\mathbf{C}+\mathbf{C}^{\mathrm{T}}\right)+\tilde{\varepsilon} \mathbf{I}\right] \mathbf{E} .
\end{aligned}
$$

Then from Eq. (23a) the symmetric part of $\mathbf{C}_{\mathrm{h}}$ is calculated as

$$
\begin{aligned}
\frac{1}{2}\left(\mathbf{C}_{\mathrm{h}}+\mathbf{C}_{\mathrm{h}}^{\mathrm{T}}\right) & =\frac{1}{2} \mathbf{E}^{\mathrm{T}}\left(\mathbf{C}+\mathbf{C}^{\mathrm{T}}\right) \mathbf{E}+\mathbf{\Omega} \\
& =\frac{1}{2} \mathbf{E}^{\mathrm{T}}\left(\mathbf{C}+\mathbf{C}^{\mathrm{T}}\right) \mathbf{E}-\mathbf{E}^{\mathrm{T}}\left(\frac{1}{2}\left(\mathbf{C}+\mathbf{C}^{\mathrm{T}}\right)+\tilde{\varepsilon} \mathbf{I}\right) \mathbf{E} \\
& =-\tilde{\varepsilon} \mathbf{E}^{\mathrm{T}} \mathbf{I} \mathbf{E} \\
& =-\tilde{\varepsilon} \mathbf{E}^{\mathrm{T}} \mathbf{E} \\
& =-\tilde{\varepsilon} \mathbf{I}_{L},
\end{aligned}
$$

which is always negative definite since $\tilde{\varepsilon}$ is positive. Thus, $\mathbf{s}$ is strongly convergence stable along the constraint surface. Third, in order to examine condition (iii) for evolutionary stability, $\mathbf{D}_{\mathrm{h}}$ is calculated from Eq. (23a) as 


$$
\begin{aligned}
\mathbf{D}_{\mathrm{h}}=\mathbf{E}^{\mathrm{T}} \mathbf{D E}+\boldsymbol{\Omega} & =\mathbf{E}^{\mathrm{T}} \mathbf{D E}-\frac{1}{2} \mathbf{E}^{\mathrm{T}}\left(\mathbf{C}+\mathbf{C}^{\mathrm{T}}\right) \mathbf{E}-\tilde{\varepsilon} \mathbf{E}^{\mathrm{T}} \mathbf{I} \mathbf{E} \\
& =\mathbf{E}^{\mathrm{T}}\left[\mathbf{D}-\frac{1}{2}\left(\mathbf{C}+\mathbf{C}^{\mathrm{T}}\right)\right] \mathbf{E}-\tilde{\varepsilon} \mathbf{I}_{L} \\
& =\frac{1}{2} \mathbf{E}^{\mathrm{T}}\left[\mathbf{M}+\mathbf{M}^{\mathrm{T}}\right] \mathbf{E}-\tilde{\varepsilon} \mathbf{I}_{L} .
\end{aligned}
$$

As shown in the subsequent subsection, the first term is expressed as

$$
\frac{1}{2} \mathbf{E}^{\mathrm{T}}\left[\mathbf{M}+\mathbf{M}^{\mathrm{T}}\right] \mathbf{E}=\left(\begin{array}{ccc}
\alpha_{1} & \cdots & 0 \\
\vdots & \ddots & \vdots \\
0 & \cdots & \alpha_{L}
\end{array}\right)
$$

where $\alpha_{1}, \cdots, \alpha_{L}$ are the eigenvalues of the branching potential matrix $\mathbf{P}$. Since $\alpha_{1}>\alpha_{2}>\cdots>\alpha_{K}>0$ is assumed, $\mathbf{D}_{\mathrm{h}}$ has at least one positive eigenvalue as long as $0<\tilde{\varepsilon}<\alpha_{1}$. Thus the point $\mathbf{s}$ is evolutionarily unstable along the surface. Therefore, by choosing a sufficiently small $\tilde{\varepsilon}$, we can make the point $\mathbf{s}$ a CBP along the surface. This completes the proof.

Moreover, if $L=K$ is chosen, then $\mathbf{D}_{\mathrm{h}}$ is positive definite as long as $0<\tilde{\varepsilon}<$ $\alpha_{K}$. In this case $\mathbf{C}_{\mathrm{h}}$ and $\mathbf{D}_{\mathrm{h}}$ are negative definite and positive definite, respectively, in which case $\mathbf{s}$ is a CBP along any smooth subspace that contains $\mathbf{s}$.

\section{G.3. Derivation of Eq. (G.14)}

Because the eigenvectors $\mathbf{v}_{1}, \cdots, \mathbf{v}_{L}, \cdots, \mathbf{v}_{M}$ of $\mathbf{P}$ given by Eq. (G.1) are orthogonal, $\mathbf{E}=\left(\mathbf{e}_{1}, \cdots, \mathbf{e}_{L}\right)=\left(\mathbf{v}_{1}, \cdots, \mathbf{v}_{L}\right)$ and $\mathbf{U}=\left[\mathbf{I}-\frac{\mathbf{g g}^{\mathrm{T}}}{|\mathbf{g}|^{2}}\right]$ with $\mathbf{v}_{M}=\frac{\mathbf{g}}{|\mathbf{g}|}$ satisfy

$$
\begin{aligned}
\mathbf{U E} & =\left[\mathbf{I}-\frac{\mathbf{g g}^{\mathrm{T}}}{|\mathbf{g}|^{2}}\right]\left(\mathbf{e}_{1}, \cdots, \mathbf{e}_{L}\right) \\
& =\mathbf{E}-\frac{\mathbf{g}}{|\mathbf{g}|}\left(\mathbf{v}_{M}^{\mathrm{T}} \mathbf{v}_{1}, \ldots, \mathbf{v}_{M}^{\mathrm{T}} \mathbf{v}_{L}\right)=\mathbf{E},
\end{aligned}
$$

which gives

$$
\mathbf{E}^{\mathrm{T}} \mathbf{P E}=\frac{1}{2} \mathbf{E}^{\mathrm{T}} \mathbf{U}^{\mathrm{T}}\left[\mathbf{M}+\mathbf{M}^{\mathrm{T}}\right] \mathbf{U E}=\frac{1}{2} \mathbf{E}^{\mathrm{T}}\left[\mathbf{M}+\mathbf{M}^{\mathrm{T}}\right] \mathbf{E} .
$$

On the other hand, $\mathbf{E}^{\mathrm{T}} \mathbf{P E}$ is transformed by Eq. (G.1) as 


$$
\begin{aligned}
\mathbf{E}^{\mathrm{T}} \mathbf{P E} & =\mathbf{E}^{\mathrm{T}}(\mathbf{E}, \mathbf{N})\left(\begin{array}{cccc}
\alpha_{1} & \cdots & \cdots & 0 \\
\vdots & \ddots & \ddots & \vdots \\
\vdots & \ddots & \alpha_{M-1} & \vdots \\
0 & \cdots & \cdots & 0
\end{array}\right)\left(\begin{array}{c}
\mathbf{E}^{\mathrm{T}} \\
\vdots \\
\mathbf{N}^{\mathrm{T}}
\end{array}\right) \mathbf{E} \\
& =\left(\begin{array}{lllll}
\mathbf{I}_{L} & \mathbf{0}_{(L, M-L)}
\end{array}\right)\left(\begin{array}{cccc}
\alpha_{1} & \cdots & \cdots & 0 \\
\vdots & \ddots & \ddots & \vdots \\
\vdots & \ddots & \alpha_{M-1} & \vdots \\
0 & \cdots & \cdots & 0
\end{array}\right)\left(\begin{array}{c}
\mathbf{I}_{L} \\
\mathbf{0}_{(M-L, L)}
\end{array}\right) \\
& =\left(\begin{array}{ccc}
\alpha_{1} & \cdots & 0 \\
\vdots & \ddots & \vdots \\
0 & \cdots & \alpha_{L}
\end{array}\right),
\end{aligned}
$$

where $\mathbf{N}=\left(\mathbf{v}_{L+1}, \cdots, \mathbf{v}_{M}\right), \mathbf{0}_{(L, M-L)}$ and $\mathbf{0}_{(M-L, L)}$ are $L$-by- $(M-L)$ and $(M-L)$ by- $L$ zero matrices. By combining Eqs. (G.16) and (G.17), we obtain Eq. (G.14).

\section{Appendix H: Derivatives in Example 2}

The first and second derivatives of $F\left(\mathbf{s}^{\prime} ; \mathbf{s}^{\circ}\right)$ are calculated as

$$
\begin{aligned}
& \nabla_{\mathbf{s}^{\prime}}^{\mathrm{T}} F\left(\mathbf{s}^{\prime} ; \mathbf{s}^{\circ}\right)=K\left(\mathbf{s}^{\circ}\right)\left[-\frac{\nabla_{\mathbf{s}^{\prime}}^{\mathrm{T}} \alpha\left(\mathbf{s} ; \mathbf{s}^{\circ}\right)}{K\left(\mathbf{s}^{\prime}\right)}+\frac{\alpha\left(\mathbf{s}^{\prime} ; \mathbf{s}^{\circ}\right) \nabla_{\mathbf{s}^{\prime}}^{\mathrm{T}} K\left(\mathbf{s}^{\prime}\right)}{K\left(\mathbf{s}^{\prime}\right)^{2}}\right] \\
& =\frac{K\left(\mathbf{s}^{\circ}\right)}{K\left(\mathbf{s}^{\prime}\right)}\left[-\nabla_{\mathbf{s}^{\prime}}^{\mathrm{T}} \alpha\left(\mathbf{s}^{\prime} ; \mathbf{s}^{\circ}\right)+\alpha\left(\mathbf{s}^{\prime} ; \mathbf{s}^{\circ}\right) \nabla_{\mathbf{s}^{\prime}}^{\mathrm{T}} \ln K\left(\mathbf{s}^{\prime}\right)\right], \\
& \nabla_{\mathbf{s}^{\prime}} \nabla_{\mathbf{s}^{\prime}}^{\mathrm{T}} F\left(\mathbf{s}^{\prime} ; \mathbf{s}^{\circ}\right)=-\frac{K\left(\mathbf{s}^{\circ}\right)}{K\left(\mathbf{s}^{\prime}\right)^{2}} \nabla_{\mathbf{s}^{\prime}} K\left(\mathbf{s}^{\prime}\right)\left[-\nabla_{\mathbf{s}^{\prime}}^{\mathrm{T}} \alpha\left(\mathbf{s}^{\prime} ; \mathbf{s}^{\circ}\right)+\alpha\left(\mathbf{s}^{\prime} ; \mathbf{s}^{\circ}\right) \nabla_{\mathbf{s}^{\prime}}^{\mathrm{T}} \ln K\left(\mathbf{s}^{\prime}\right)\right] \\
& +\frac{K\left(\mathbf{s}^{\circ}\right)}{K\left(\mathbf{s}^{\prime}\right)}\left[-\nabla_{\mathbf{s}^{\prime}} \nabla_{\mathbf{s}^{\prime}}^{\mathrm{T}} \alpha\left(\mathbf{s}^{\prime} ; \mathbf{s}^{\circ}\right)+\nabla_{\mathbf{s}^{\prime}} \alpha\left(\mathbf{s}^{\prime} ; \mathbf{s}^{\circ}\right) \nabla_{\mathbf{s}^{\prime}}^{\mathrm{T}} \ln K\left(\mathbf{s}^{\prime}\right)+\alpha\left(\mathbf{s}^{\prime} ; \mathbf{s}^{\circ}\right) \nabla_{\mathbf{s}^{\prime}} \nabla_{\mathbf{s}^{\mathrm{T}}}^{\mathrm{T}} \ln K\left(\mathbf{s}^{\prime}\right)\right], \\
& \nabla_{\mathbf{s}^{\circ}} \nabla_{\mathbf{s}^{\prime}}^{\mathrm{T}} F\left(\mathbf{s}^{\prime} ; \mathbf{s}^{\circ}\right)=\frac{\nabla_{\mathbf{s}^{\circ}} K\left(\mathbf{s}^{\circ}\right)}{K\left(\mathbf{s}^{\prime}\right)}\left[-\nabla_{\mathbf{s}^{\prime}}^{\mathrm{T}} \alpha\left(\mathbf{s}^{\prime} ; \mathbf{s}^{\circ}\right)+\alpha\left(\mathbf{s}^{\prime} ; \mathbf{s}^{\circ}\right) \nabla_{\mathbf{s}^{\prime}}^{\mathrm{T}} \ln K\left(\mathbf{s}^{\prime}\right)\right] \\
& +\frac{K\left(\mathbf{s}^{\circ}\right)}{K\left(\mathbf{s}^{\prime}\right)}\left[-\nabla_{\mathbf{s}^{\circ}} \nabla_{\mathbf{s}^{\prime}}^{\mathrm{T}} \alpha\left(\mathbf{s}^{\prime} ; \mathbf{s}^{\circ}\right)+\nabla_{\mathbf{s}^{\circ}} \alpha\left(\mathbf{s}^{\prime} ; \mathbf{s}^{\circ}\right) \nabla_{\mathbf{s}^{\mathrm{T}}}^{\mathrm{T}} \ln K\left(\mathbf{s}^{\prime}\right)\right] .
\end{aligned}
$$

We obtain $\mathbf{g}, \mathbf{D}$, and $\mathbf{C}$ at $\mathbf{s}$ by exploiting $\alpha(\mathbf{s} ; \mathbf{s})=1, \nabla_{\mathbf{s}^{\prime}} \alpha(\mathbf{s} ; \mathbf{s})=\nabla_{\mathbf{s}^{\circ}} \alpha(\mathbf{s} ; \mathbf{s})=$ 0 , and $\nabla_{\mathbf{s}^{\circ}} \nabla_{\mathbf{s}^{\prime}}^{\mathrm{T}} \alpha(\mathbf{s} ; \mathbf{s})=-\nabla_{\mathbf{s}^{\prime}} \nabla_{\mathbf{s}^{\prime}}^{\mathrm{T}} \alpha(\mathbf{s} ; \mathbf{s})$ (because $\alpha\left(\mathbf{s}^{\prime} ; \mathbf{s}^{\circ}\right)=\tilde{\alpha}\left(\mathbf{s}^{\prime}-\mathbf{s}^{\circ}\right)$ ), as 


$$
\begin{aligned}
& \mathbf{g}=\nabla_{\mathbf{s}^{\prime}} F(\mathbf{s} ; \mathbf{s})=\nabla \ln K(\mathbf{s}), \\
& \mathbf{D}=\nabla_{\mathbf{s}^{\prime}} \nabla_{\mathbf{s}^{\prime}}^{\mathrm{T}} F(\mathbf{s} ; \mathbf{s}) \\
& =-\nabla_{\mathbf{s}^{\prime}} \nabla_{\mathbf{s}^{\prime}}^{\mathrm{T}} \alpha(\mathbf{s} ; \mathbf{s})+\nabla \nabla^{\mathrm{T}} \ln K(\mathbf{s})-\nabla \ln K(\mathbf{s}) \nabla^{\mathrm{T}} \ln K(\mathbf{s}) \\
& =-\nabla_{\mathbf{s}^{\prime}} \nabla_{\mathbf{s}^{\prime}}^{\mathrm{T}} \alpha(\mathbf{s} ; \mathbf{s})+\nabla \nabla^{\mathrm{T}} \ln K(\mathbf{s})-\mathbf{g g}^{T}, \\
& \mathbf{C}=\nabla_{\mathbf{s}^{\prime}} \nabla_{\mathbf{s}^{\prime}}^{\mathrm{T}} F(\mathbf{s} ; \mathbf{s})+\nabla_{\mathbf{s}^{\circ}} \nabla_{\mathbf{s}^{\prime}}^{\mathrm{T}} F(\mathbf{s} ; \mathbf{s}) \\
& =\left[-\nabla_{\mathbf{s}^{\prime}} \nabla_{\mathbf{s}^{\prime}}^{\mathrm{T}} \alpha\left(\mathbf{s}^{\prime} ; \mathbf{s}^{\prime}\right)+\nabla \nabla^{\mathrm{T}} \ln K(\mathbf{s})-\nabla \ln K(\mathbf{s}) \nabla^{\mathrm{T}} \ln K(\mathbf{s})\right] \\
& +\left[-\nabla_{\mathbf{s}^{\circ}} \nabla_{\mathbf{s}^{\prime}}^{\mathrm{T}} \alpha(\mathbf{s} ; \mathbf{s})+\nabla^{\mathrm{T}} \ln K(\mathbf{s}) \nabla^{\mathrm{T}} \ln K(\mathbf{s})\right] \\
& =\nabla \nabla^{\mathrm{T}} \ln K(\mathbf{s}) .
\end{aligned}
$$




\section{Table 1 Ito and Sasaki}

\begin{tabular}{|c|c|c|c|}
\hline & $\begin{array}{l}\text { Original fitness for mutant } \\
\mathbf{s}^{\prime} \text { against resident } \mathbf{s}^{\circ} \\
F\left(\mathbf{s}^{\prime} ; \mathbf{s}^{\circ}\right)\end{array}$ & $\begin{array}{l}\text { Lagrange fitness function } \\
\begin{aligned} F_{\mathrm{L}}\left(\mathbf{s}^{\prime} ; \mathbf{s}^{\circ} ; \lambda\right)=F\left(\mathbf{s}^{\prime} ; \mathbf{s}^{\circ}\right) \\
-\lambda\left[h\left(\mathbf{s}^{\prime}\right)-h\left(\mathbf{s}^{\circ}\right)\right]\end{aligned}\end{array}$ & $\begin{array}{l}\text { Fitness along } \\
\text { constraint curve }\end{array}$ \\
\hline $\begin{array}{l}\text { Gradient } \\
\text { (Evolutionary } \\
\text { singularity) }\end{array}$ & $\begin{array}{l}\mathbf{g} \\
=\nabla_{\mathbf{s}^{\prime}} F(\mathbf{s} ; \mathbf{s})\end{array}$ & $\begin{array}{l}\mathbf{g}_{\mathrm{L}} \\
=\nabla_{\mathbf{s}^{\prime}} F_{\mathrm{L}}\left(\mathbf{s} ; \mathbf{s} ; \lambda_{\mathbf{s}}\right) \\
=\mathbf{e}\left[\mathbf{e}^{\mathrm{T}} \mathbf{g}\right]\end{array}$ & $\begin{array}{l}g_{\mathrm{h}} \\
=\mathbf{e}^{\mathrm{T}} \mathbf{g}_{\mathrm{L}} \\
=\mathbf{e}^{\mathrm{T}} \mathbf{g}\end{array}$ \\
\hline $\begin{array}{l}\text { Gradient variability } \\
\text { (Convergence } \\
\text { stability) }\end{array}$ & $\begin{array}{l}\mathbf{C} \\
=\left(\nabla_{\mathbf{s}^{\prime}} \nabla_{\mathbf{s}^{\prime}}^{\mathrm{T}}+\nabla_{\mathbf{s}^{\circ}} \nabla_{\mathbf{s}^{\prime}}^{\mathrm{T}}\right) F(\mathbf{s} ; \mathbf{s})\end{array}$ & $\begin{array}{l}\mathbf{C}_{\mathrm{L}} \\
=\left(\nabla_{\mathbf{s}^{\prime}} \nabla_{\mathbf{s}^{\prime}}^{\mathrm{T}}+\nabla_{\mathbf{s}^{\circ}} \nabla_{\mathbf{s}^{\prime}}^{\mathrm{T}}\right) F_{\mathrm{L}}\left(\mathbf{s} ; \mathbf{s} ; \lambda_{\mathbf{s}}\right)\end{array}$ & $\begin{array}{l}C_{\mathrm{h}} \\
=\mathbf{e}^{\mathrm{T}} \mathbf{C}_{\mathrm{L}} \mathbf{e} \\
=\mathbf{e}^{\mathrm{T}} \mathbf{C e}+\Omega\end{array}$ \\
\hline $\begin{array}{l}\text { Curvature } \\
\text { (Evolutionary } \\
\text { stability) }\end{array}$ & $\begin{array}{l}\mathbf{D} \\
=\nabla_{\mathbf{s}^{\prime}} \nabla_{\mathbf{s}^{\prime}}^{\mathrm{T}} F(\mathbf{s} ; \mathbf{s})\end{array}$ & $\begin{array}{l}\mathbf{D}_{\mathrm{L}} \\
=\nabla_{\mathbf{s}^{\prime}} \nabla_{\mathbf{s}^{\prime}}^{\mathrm{T}} F_{\mathrm{L}}\left(\mathbf{s} ; \mathbf{s} ; \lambda_{\mathbf{s}}\right)\end{array}$ & $\begin{array}{l}D_{\mathrm{h}} \\
=\mathbf{e}^{\mathrm{T}} \mathbf{D}_{\mathrm{L}} \mathbf{e} \\
=\mathbf{e}^{\mathrm{T}} \mathbf{D e}+\Omega\end{array}$ \\
\hline
\end{tabular}

Table 1. Local fitness landscape at an arbitrary point $\mathbf{s}$ along constraint curve $h\left(\mathbf{s}^{\prime}\right)=0$ in two-dimensional trait space $\mathbf{s}=(x, y)^{\mathrm{T}}($ Section 3$) . \lambda_{\mathbf{s}}=$ $\left(\nabla h(\mathbf{s}) \cdot \nabla_{\mathbf{s}^{\prime}} F(\mathbf{s} ; \mathbf{s})\right) /|\nabla h(\mathbf{s})|^{2}, \Omega=\mathbf{e}^{\mathrm{T}}\left[\lambda_{\mathbf{s}} \nabla \nabla^{\mathrm{T}} h(\mathbf{s})\right] \mathbf{e}$, and $\mathbf{e}$ is the tangent vector of the constraint curve at $\mathbf{s}$. 


\section{Table 2 Ito and Sasaki}

\begin{tabular}{|c|c|c|c|}
\hline & $\begin{array}{l}\text { Original fitness for mutant } \\
\mathbf{s}^{\prime} \text { against resident } \mathbf{s}^{\circ} \\
F\left(\mathbf{s}^{\prime} ; \mathbf{s}^{\circ}\right)\end{array}$ & $\begin{array}{l}\text { Lagrange fitness function } \\
F_{\mathrm{L}}\left(\mathbf{s}^{\prime} ; \mathbf{s}^{\circ} ; \boldsymbol{\lambda}\right)=F\left(\mathbf{s}^{\prime} ; \mathbf{s}^{\circ}\right) \\
\quad-\sum_{j=L+1}^{M} \lambda_{j}\left[h_{j}\left(\mathbf{s}^{\prime}\right)-h_{j}\left(\mathbf{s}^{\circ}\right)\right]\end{array}$ & $\begin{array}{l}\text { Fitness along } \\
\text { constraint surface }\end{array}$ \\
\hline $\begin{array}{l}\text { Gradient } \\
\text { (Evolutionary } \\
\text { singularity) }\end{array}$ & $\begin{array}{l}\mathbf{g} \\
=\nabla_{\mathbf{s}^{\prime}} F(\mathbf{s} ; \mathbf{s})\end{array}$ & $\begin{array}{l}\mathbf{g}_{\mathrm{L}} \\
=\nabla_{\mathbf{s}^{\prime}} F_{\mathrm{L}}\left(\mathbf{s} ; \mathbf{s} ; \boldsymbol{\lambda}_{\mathbf{s}}\right) \\
=\mathbf{E E}^{\mathrm{T}} \mathbf{g}\end{array}$ & $\begin{array}{l}\mathbf{g}_{\mathrm{h}} \\
=\mathbf{E}^{\mathrm{T}} \mathbf{g}_{\mathrm{L}} \\
=\mathbf{E}^{\mathrm{T}} \mathbf{g}\end{array}$ \\
\hline $\begin{array}{l}\text { Gradient variability } \\
\text { (Convergence } \\
\text { stability) }\end{array}$ & $\begin{array}{l}\mathbf{C} \\
=\left(\nabla_{\mathbf{s}^{\prime}} \nabla_{\mathbf{s}^{\prime}}^{\mathrm{T}}+\nabla_{\mathbf{s}^{\circ}} \nabla_{\mathbf{s}^{\prime}}^{\mathrm{T}}\right) F(\mathbf{s} ; \mathbf{s})\end{array}$ & $\begin{array}{l}\mathbf{C}_{\mathrm{L}} \\
=\left(\nabla_{\mathbf{s}^{\prime}} \nabla_{\mathbf{s}^{\prime}}^{\mathrm{T}}+\nabla_{\mathbf{s}^{\circ}} \nabla_{\mathbf{s}^{\prime}}^{\mathrm{T}}\right) F_{\mathrm{L}}\left(\mathbf{s} ; \mathbf{s} ; \boldsymbol{\lambda}_{\mathbf{s}}\right)\end{array}$ & $\begin{array}{l}\mathbf{C}_{\mathrm{h}} \\
=\mathbf{E}^{\mathrm{T}} \mathbf{C}_{\mathrm{L}} \mathbf{E} \\
=\mathbf{E}^{\mathrm{T}} \mathbf{C E}+\mathbf{\Omega}\end{array}$ \\
\hline $\begin{array}{l}\text { Curvature } \\
\text { (Evolutionary } \\
\text { stability) }\end{array}$ & $\begin{array}{l}\mathbf{D} \\
=\nabla_{\mathbf{s}^{\prime}} \nabla_{\mathbf{s}^{\prime}}^{\mathrm{T}} F(\mathbf{s} ; \mathbf{s})\end{array}$ & $\begin{array}{l}\mathbf{D}_{\mathrm{L}} \\
=\nabla_{\mathbf{s}^{\prime}} \nabla_{\mathbf{s}^{\prime}}^{\mathrm{T}} F_{\mathrm{L}}\left(\mathbf{s} ; \mathbf{s} ; \boldsymbol{\lambda}_{\mathbf{s}}\right)\end{array}$ & $\begin{array}{l}\mathbf{D}_{\mathrm{h}} \\
=\mathbf{E}^{\mathrm{T}} \mathbf{D}_{\mathrm{L}} \mathbf{E} \\
=\mathbf{E}^{\mathrm{T}} \mathbf{D E}+\mathbf{\Omega}\end{array}$ \\
\hline
\end{tabular}

Table 2. Local fitness landscape at an arbitrary point $\mathbf{s}$ along $L$-dimensional constraint surface $\left(h_{L+1}\left(\mathbf{s}^{\prime}\right), \cdots, h_{M}\left(\mathbf{s}^{\prime}\right)\right)=\mathbf{0}$ in $M$-dimensional trait space $\mathbf{s}=$ $\left(x_{1}, \cdots, x_{M}\right)^{\mathrm{T}}$ (Section 4). $\boldsymbol{\lambda}_{\mathbf{s}}=\mathbf{B}^{+} \mathbf{g}=\left[\mathbf{B}^{\mathrm{T}} \mathbf{B}\right]^{-1} \mathbf{B}^{\mathrm{T}} \mathbf{g}$ with $\mathbf{B}=$ $\left(\nabla h_{L+1}(\mathbf{s}), \cdots, \nabla h_{M}(\mathbf{s})\right), \boldsymbol{\Omega}=-\sum_{j=L+1}^{M} \lambda_{\mathbf{s} j} \mathbf{E}^{\mathrm{T}} \nabla \nabla^{\mathrm{T}} h_{j}(\mathbf{s}) \mathbf{E}$, and $\mathbf{E}=\left(\mathbf{e}_{1}, \cdots, \mathbf{e}_{L}\right)$ are the orthogonal base vectors of the tangent plane of the constraint surface at $\mathbf{s}$. 
Figure 1 Ito and Sasaki

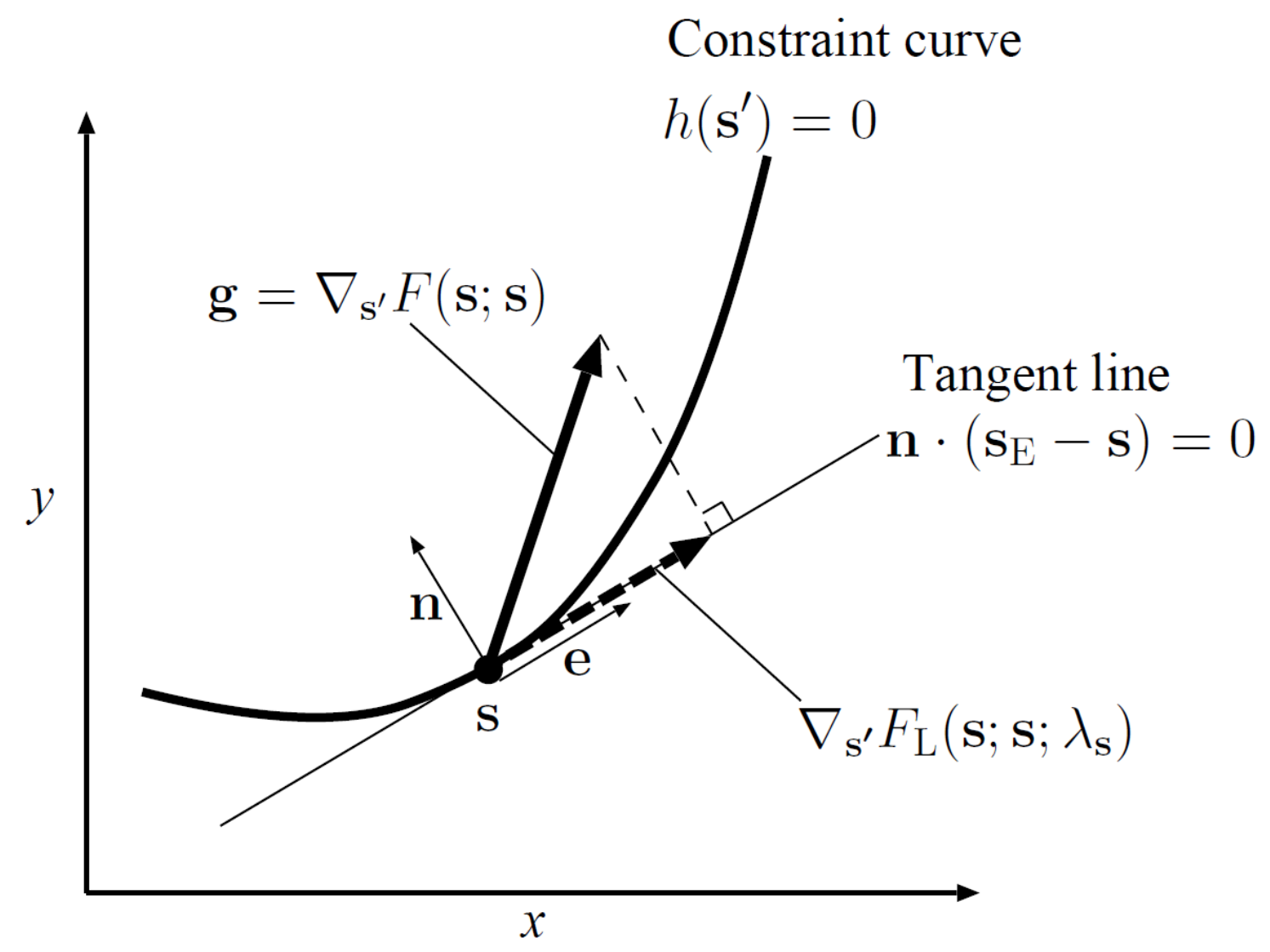


Figure 2 Ito and Sasaki

(a)

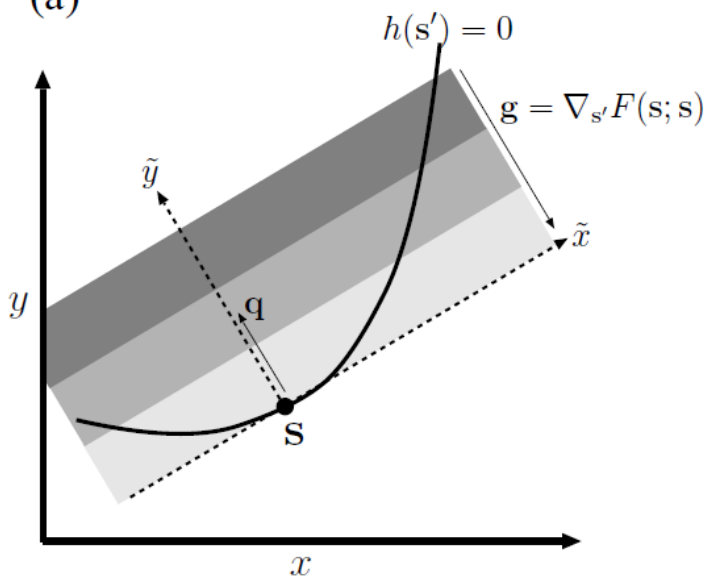

(c)

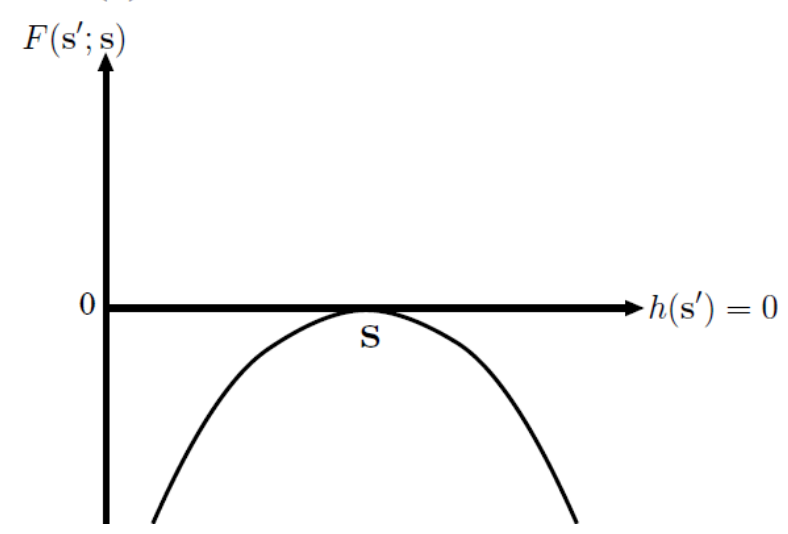

(b)

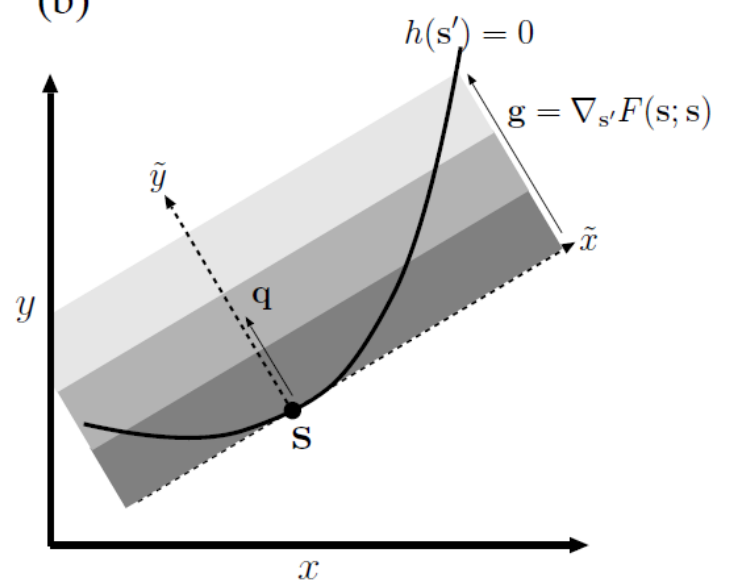

(d)

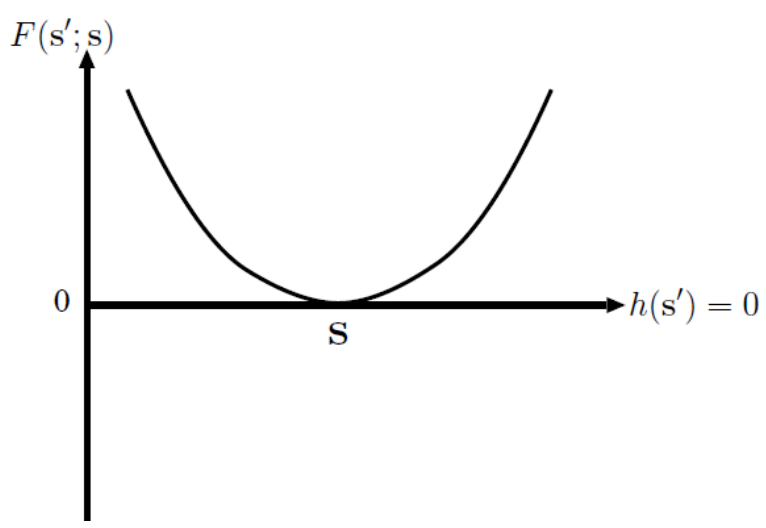


Figure 3 Ito and Sasaki
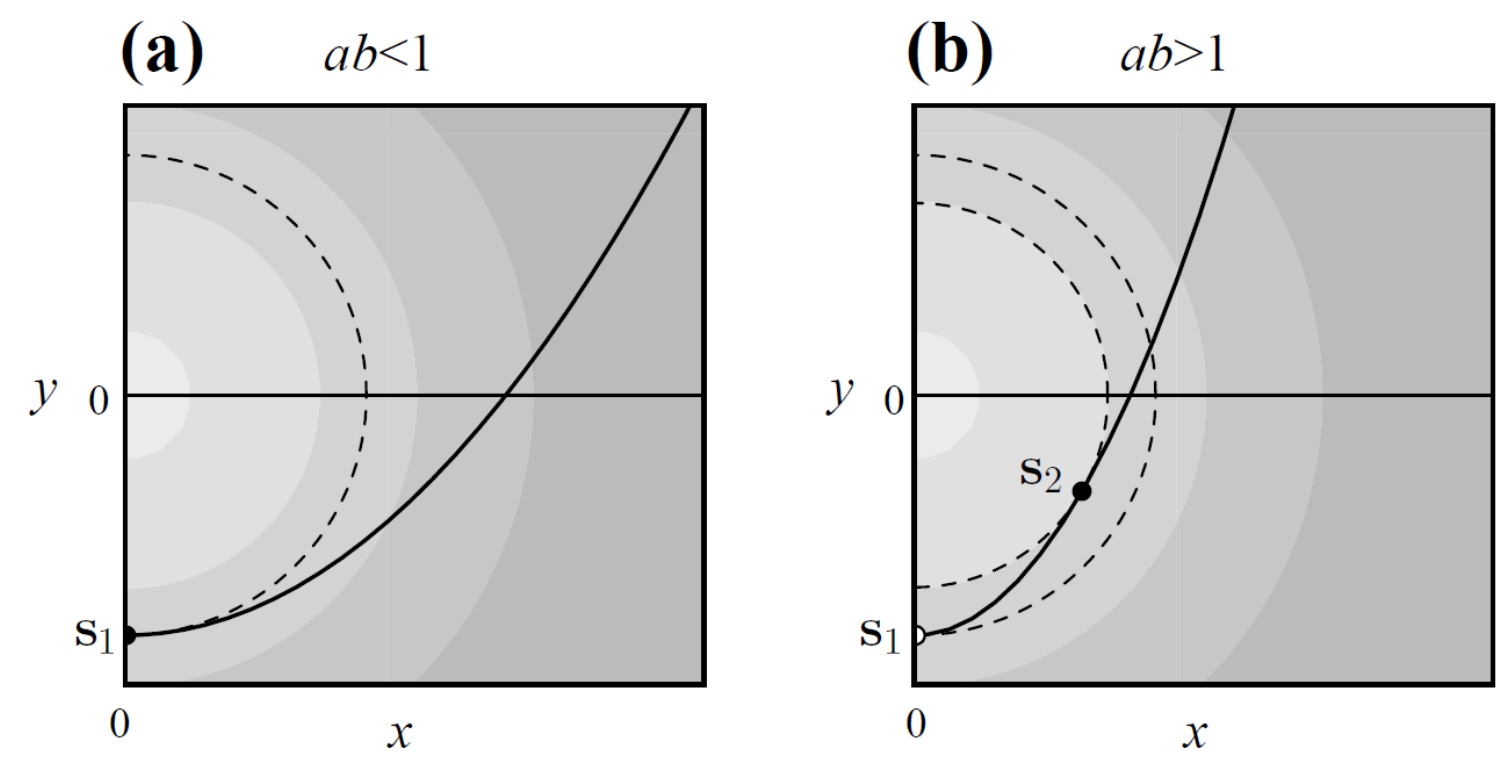


\section{Figure 4 Ito and Sasaki}

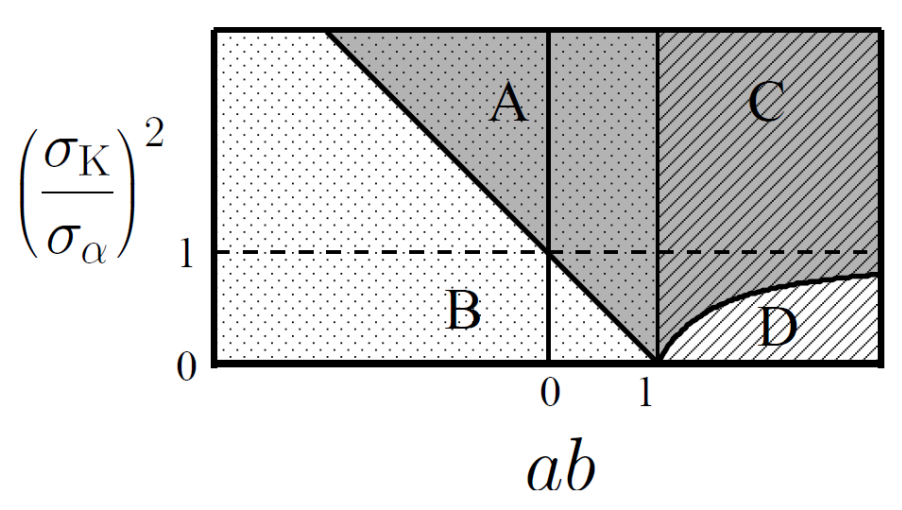

Convergence stability

. Only $\mathbf{S}_{1}$ stable ( $\mathbf{S}_{2}$ absence)

Only $\mathbf{S}_{2}$ stable

Evolutionary stability

Unstable

Stable 


\section{Figure 5 Ito and Sasaki}

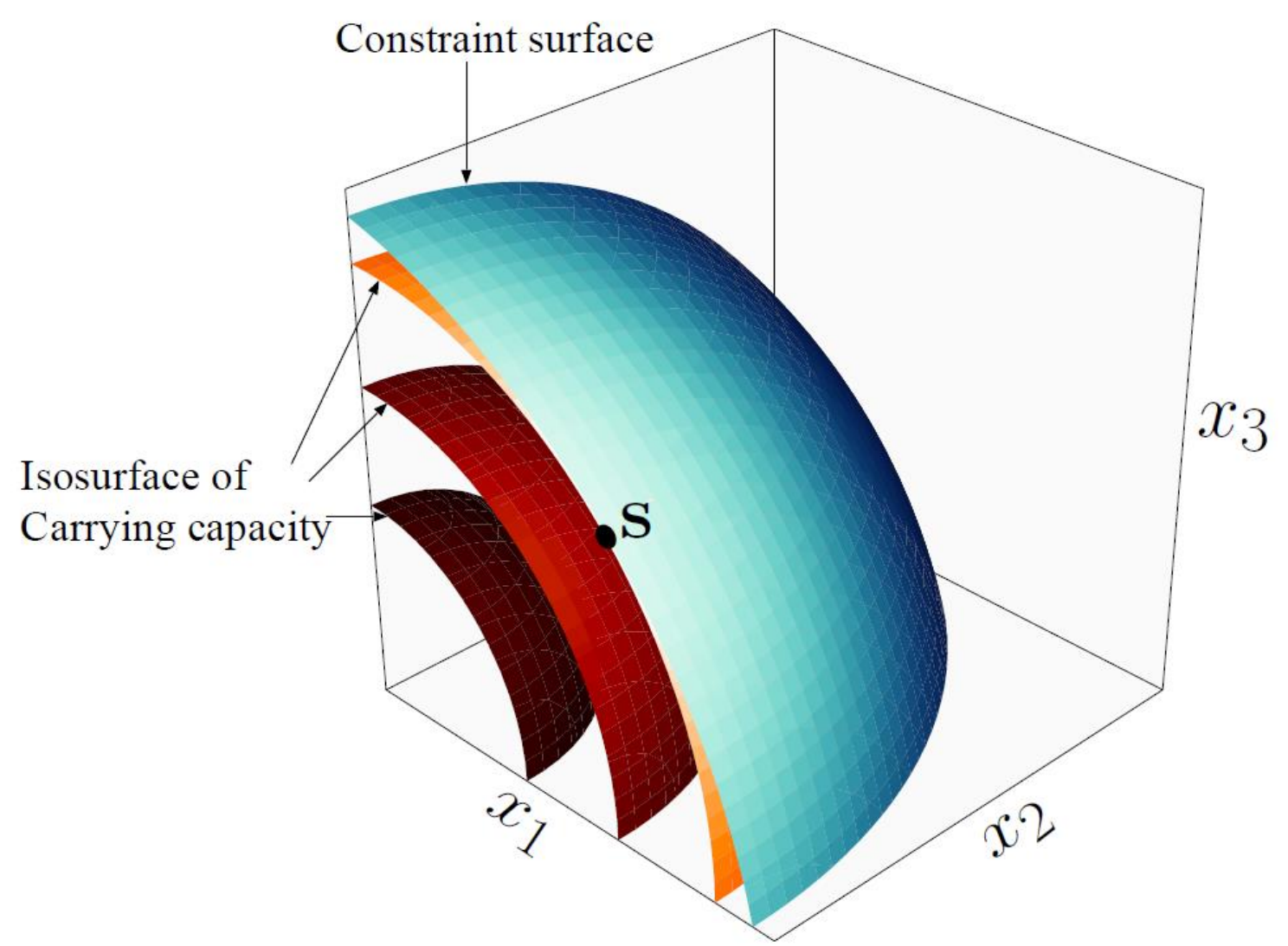

